\title{
THE MONGE-AMPÈRE EQUATION AND ITS LINK TO OPTIMAL TRANSPORTATION
}

\author{
GUIDO DE PHILIPPIS AND ALESSIO FIGALLI
}

\begin{abstract}
We survey old and new regularity theory for the Monge-Ampère equation, show its connection to optimal transportation, and describe the regularity properties of a general class of Monge-Ampère type equations arising in that context.
\end{abstract}

\section{Contents}

1. Introduction

2. The classical Monge-Ampère equation

2.1. Alexandrov solutions and regularity results

2.2. The continuity method and existence of smooth solutions

2.3. Interior estimates and regularity of weak solutions.

2.4. Further regularity results for weak solutions

2.5. An application: global existence for the semigeostrophic equations

3. The optimal transport problem

3.1. The quadratic cost on $\mathbb{R}^{n}$

3.2. Regularity theory for the quadratic cost: Brenier vs. Alexandrov solutions

3.3. The partial transport problem

3.4. The case of a general cost

4. A class of Monge-Ampère type equations

4.1. A geometric interpretation of the MTW condition

4.2. Regularity results

4.3. The case of Riemannian manifolds

4.4. MTW vs. cut-locus

4.5. Partial regularity

5. Open problems and further perspectives

5.1. General prescribed Jacobian equations

5.2. Open problems

Acknowledgments 


\section{INTRODUCTION}

The Monge-Ampère equation arises in several problems from analysis and geometry. In its classical form this equation is given by

$$
\operatorname{det} D^{2} u=f(x, u, \nabla u) \quad \text { in } \Omega,
$$

where $\Omega \subset \mathbb{R}^{n}$ is some open set, $u: \Omega \rightarrow \mathbb{R}$ is a convex function, and $f: \Omega \times \mathbb{R} \times \mathbb{R}^{n} \rightarrow$ $\mathbb{R}^{+}$is given. In other words, the Monge-Ampère equation prescribes the product of the eigenvalues of the Hessian of $u$, in contrast with the "model" elliptic equation $\Delta u=f$ which prescribes their sum. As we shall explain later, the convexity of the solution $u$ is a necessary condition to make the equation degenerate elliptic, and so to hope for regularity results.

The prototype equation where the Monge-Ampère equation appears is the "prescribed Gaussian curvature equation": if we take $f=K(x)\left(1+|\nabla u|^{2}\right)^{(n+2) / 2}$, then (1.1) corresponds to imposing that the Gaussian curvature of the graph of $u$ at the point $(x, u(x))$ is equal to $K(x)$. Another classical instance where the Monge-Ampère equation arises is affine geometry, more precisely in the "affine sphere problem" and the "affine maximal surfaces" problem (see for instance [27, 29, 95, 104 106 ). The Monge-Ampère equation (1.1) also arises in meteorology and fluid mechanics: for instance, in the semigeostrophic equations it is coupled with a transport equation (see Section 2.5 below).

As we shall see later, in the optimal transportation problem the study of MongeAmpère type equation of the form

$$
\operatorname{det}\left(D^{2} u-\mathcal{A}(x, \nabla u)\right)=f(x, u, \nabla u)
$$

plays a key role in understanding the regularity (or singularity) of optimal transport maps.

More in general, Monge-Ampère type equations of the form

$$
\operatorname{det}\left(D^{2} u-\mathcal{A}(x, u, \nabla u)\right)=f(x, u, \nabla u),
$$

have found applications in several other problems, such as isometric embeddings, reflector shape design, and in the study of special Lagrangian submanifolds, prescribing Weingarten curvature, and in complex geometry on toric manifolds (see for instance the survey paper [107] for more details on these geometric applications).

The aim of this article is to describe the general regularity theory for the MongeAmpère equation (1.1), show its connections with the optimal transport problem with quadratic cost, introduce the more general class of Monge-Ampère type equations (1.2) which arise when the cost is not quadratic, and show some regularity results for this class of equations.

\section{The Classical Monge-Ampère equation}

The Monge-Ampère equation (1.1) draws its name from its initial formulation in two dimensions by the French mathematicians Monge [92] and Ampère [10] about two hundred years ago. Before describing some history of the regularity theory for this equation, we first explain why convexity of the solution is a natural condition to hope for regularity results.

Remark 2.1 (On the degenerate ellipticity of the Monge-Ampère equation on convex functions). Let $u: \Omega \rightarrow \mathbb{R}$ be a smooth solution of (1.1) with $f=f(x)>0$ 
smooth, and let us try to understand whether we can prove some regularity estimates. A standard technique for dealing with nonlinear equations consists in differentiating the equation solved by $u$ to obtain a linear second-order equation for its first derivatives. More precisely, let us fix a direction $e \in \mathbb{S}^{n-1}$ and differentiate (1.1) in the direction $e$ to obtain 1

$$
\operatorname{det}\left(D^{2} u\right) u^{i j} \partial_{i j} u_{e}=f_{e} \quad \text { in } \Omega,
$$

where $u^{i j}$ denotes the inverse matrix of $u_{i j}:=\left(D^{2} u\right)_{i j}$, the lower indices denote partial derivatives (thus $u_{e}:=\partial_{e} u$ ), and we are summing over repeated indices. Recalling that $\operatorname{det} D^{2} u=f>0$ by assumption, we can rewrite the above equation as

$$
a_{i j} \partial_{i j} u_{e}=\frac{f_{e}}{f} \quad \text { in } \Omega, \quad \text { where } a_{i j}:=u^{i j} .
$$

Hence, to obtain some regularity estimates on $u_{e}$, we would like the matrix $a_{i j}$ to be nonnegative definite (and if possible even positive definite) to apply elliptic regularity theory for linear equations. But for the matrix $a_{i j}=u^{i j}$ to be nonnegative definite we need $D^{2} u$ to be nonnegative definite, which is exactly the convexity assumption on $u$.

We now observe that, without any a priori bound on $D^{2} u$, the matrix $a_{i j}$ may have arbitrarily small eigenvalues and this is why one says that (1.1) is "degenerate elliptic".

Notice however that if one can show that $\operatorname{Id} / C \leq D^{2} u \leq C$ Id inside $\Omega$ for some constant $C>0$, then $\mathrm{Id} / C \leq a_{i j} \leq C \mathrm{Id}$ and the linearized equation (2.1) becomes uniformly elliptic. For this reason, proving the bound $\operatorname{Id} / C \leq D^{2} u \leq C \operatorname{Id}$ is one of the key steps for the regularity of solutions to (1.1).

In this regard we remark that if we assume that $u$ is convex and $f \geq c_{0}>0$ for some constant $c_{0}$, then the product of the eigenvalues of $D^{2} u$ is bounded away from zero, and to obtain the estimate Id $/ C \leq D^{2} u \leq C$ Id it is actually enough to prove only the upper bound $\left|D^{2} u\right| \leq \bar{C}$ for some constant $\bar{C}$. Indeed, the latter estimate implies that all eigenvalues are bounded. Therefore, since the eigenvalues are nonnegative and their product is greater than $c_{0}$, each of them must also be uniformly bounded away from zero.

We now give a brief overview on the existence and regularity theory for the Monge-Ampère equation.

The first notable results are by Minkowski [89, 90] who proved the existence of a weak solution to the "prescribed Gaussian curvature equation" (now called the "Minkowski problem") by approximation by convex polyhedra with given face areas. Using convex polyhedra with given generalized curvatures at the vertices, Alexandrov also proved the existence of a weak solution in all dimensions, as well as the $C^{1}$-smoothness of solutions in two dimensions [2 4].

In high dimensions, based on his earlier works, Alexandrov [5] (and also Bakelman [12] in two dimensions) introduced a notion of a generalized solution to the Monge-Ampère equation and proved the existence and uniqueness of solutions to

${ }^{1}$ To compute this equation, we use the well-known formula

$$
\left.\frac{d}{d \varepsilon}\right|_{\varepsilon=0} \operatorname{det}(A+\varepsilon B)=\operatorname{det}(A) \operatorname{tr}\left(A^{-1} B\right)
$$

for all $n \times n$-matrices $A, B$, with $A$ invertible. 
the Dirichlet problem (see Section 2.1). The treatment also lead to the AlexandrovBakelman maximum principle which plays a fundamental role in the study of nondivergence elliptic equations (see for instance [62, Section 9.8]). As we shall see in Section 2.1, the notion of weak solutions introduced by Alexandrov (now called "Alexandrov solutions") has continued to be frequently used in recent years, and much attention has been drawn to prove the smoothness of Alexandrov solutions under suitable assumptions on the right-hand side and the boundary data.

The regularity of generalized solutions in high dimensions is a very delicate problem. Pogorelov found a convex function which is not of class $C^{2}$ but satisfies the Monge-Ampère equation (1.1) inside $B_{1 / 2}$ with a positive analytic right-hand side (see (2.26) below). As we shall describe in Section 2.4 the main issue in the lack of regularity is the presence of a line segment in the graph of $u$. Indeed, Calabi [26] and Pogorelov [94] were able to prove a priori interior second derivative estimate for strictly convex solutions or for solutions which do not contain a line segment with both endpoints on boundary. By the interior regularity theory for fully nonlinear uniformly elliptic equations established by Evans 43 and Krylov [77] in the 1980s, Pogorelov's second derivative estimate implies the smoothness of strictly convex Alexandrov generalized solutions.

By the regularity theory developed by Ivochkina 68, Krylov [78, and Caffarelli, Nirenberg, and Spruck [32, using the continuity method (see Section 2.2 for a description of this method), one obtains globally smooth solutions to the Dirichlet problem. In particular, Alexandrov solutions are smooth up to the boundary, provided all given data are smooth.

In all the situations mentioned above, one assumes that $f$ is positive and sufficiently smooth. When $f$ is merely bounded away from zero and infinity, Caffarelli proved the $C^{1, \alpha}$-regularity of strictly convex solutions [18. Furthermore, when $f$ is continuous (resp. $C^{0, \alpha}$ ), Caffarelli proved by a perturbation argument the interior $W^{2, p}$-estimate for any $p>1$ (resp. $C^{2, \alpha}$-interior estimates) [17. More recently, the authors proved interior $L \log L$-estimates on $D^{2} u$ when $f$ is merely bounded away from zero and infinity [35, and together with Savin they improved this result showing that $u \in W_{\text {loc }}^{2,1+\varepsilon}$ 40.

In the next sections we will give a description of these results.

2.1. Alexandrov solutions and regularity results. In his study of the Minkowski problem, Alexandrov introduced a notion of weak solutions to the MongeAmpère equation allowing him to give a meaning to the Gaussian curvature of nonsmooth convex sets.

Let us first recall that, given an open convex domain $\Omega$, the subdifferential of a convex function $u: \Omega \rightarrow \mathbb{R}$ is given by

$$
\partial u(x):=\left\{p \in \mathbb{R}^{n}: u(y) \geq u(x)+p \cdot(y-x) \quad \forall y \in \Omega\right\} .
$$

One then defines the Monge-Ampère measure of $u$ as

$$
\mu_{u}(E):=|\partial u(E)| \quad \text { for every Borel set } E \subset \Omega,
$$

where

$$
\partial u(E):=\bigcup_{x \in E} \partial u(x)
$$

and $|\cdot|$ denotes the Lebesgue measure. It is possible to show that $\mu_{u}$ is a Borel measure (see [64, Theorem 1.1.13]). Note that, in case $u \in C^{2}(\Omega)$, the change of 
variable formula gives

$$
|\partial u(E)|=|\nabla u(E)|=\int_{E} \operatorname{det} D^{2} u(x) d x \quad \text { for every Borel set } E \subset \Omega,
$$

therefore

$$
\mu_{u}=\operatorname{det} D^{2} u(x) d x \quad \text { inside } \Omega .
$$

Example 2.2. Let $u(x)=|x|^{2} / 2+\left|x_{1}\right|$, then (writing $x=\left(x_{1}, x^{\prime}\right) \in \mathbb{R} \times \mathbb{R}^{n-1}$ )

$$
\partial u(x)= \begin{cases}\left\{x+e_{1}\right\} & \text { if } x_{1}>0 \\ \left\{x-e_{1}\right\} & \text { if } x_{1}<0 \\ \left\{\left(t, x^{\prime}\right):|t| \leq 1\right\} & \text { if } x_{1}=0 .\end{cases}
$$

Thus $\mu_{u}=d x+\mathcal{H}^{n-1}\left\llcorner\left\{x_{1}=0\right\}\right.$, where $\mathcal{H}^{n-1}$ denotes the $(n-1)$-dimensional Hausdorff measure.

Definition 2.3 (Alexandrov solutions). Given an open convex set $\Omega$ and a Borel measure $\mu$ on $\Omega$, a convex function $u: \Omega \rightarrow \mathbb{R}$ is called an Alexandrov solution to the Monge-Ampère equation

$$
\operatorname{det} D^{2} u=\mu \text {, }
$$

if $\mu=\mu_{u}$ as Borel measures.

When $\mu=f d x$ we will simply say that $u$ solves

$$
\operatorname{det} D^{2} u=f \text {. }
$$

In the same way, when we write $\operatorname{det} D^{2} u \geq \lambda(\leq 1 / \lambda)$, we mean that $\mu_{u} \geq \lambda d x$ $(\leq 1 / \lambda d x)$.

One nice property of the Monge-Ampère measure is that it is stable under uniform convergence (see [64, Lemma 1.2.3]):

Proposition 2.4. Let $u_{k}: \Omega \rightarrow \mathbb{R}$ be a sequence of convex functions converging locally uniformly to $u$. Then the associated Monge-Ampère measures $\mu_{u_{k}}$ weakly* converge to $\mu_{u}$ (i.e., in duality with the space of continuous functions compactly supported in $\Omega$ ).

We now describe how to prove existence, uniqueness, and stability of Alexandrov solutions for the Dirichlet problem.

As we shall see, the existence of a weak solution is proved by an approximation and Perron-type argument, and for this it is actually useful to know a priori that solutions, if they exist, are unique and stable. We begin with the following simple lemma.

Lemma 2.5. Let $u$ and $v$ be convex functions in $\mathbb{R}^{n}$. If $E$ is an open and bounded set such that $u=v$ on $\partial E$ and $u \leq v$ in $E$, then

$$
\partial u(E) \supset \partial v(E) .
$$

In particular $\mu_{u}(E) \geq \mu_{v}(E)$.

Proof. Let $p \in \partial v(x)$ for some $x \in U$. Geometrically, this means that the plane

$$
y \mapsto v(x)+p \cdot(y-x)
$$

is a supporting plane to $v$ at $x$, that is, it touches from below the graph of $v$ at the point $(x, v(x))$. Moving this plane down until it lies below $u$ and then lifting it 


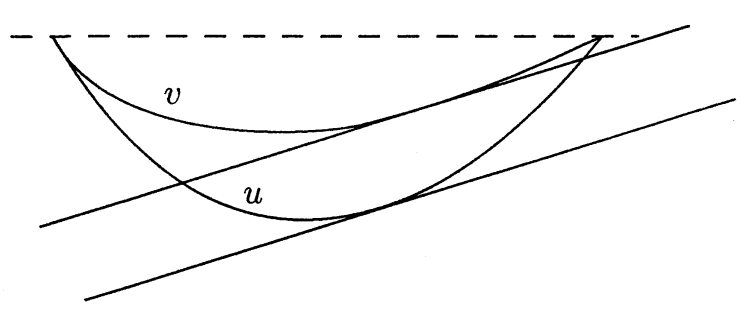

Figure 2.1. Moving down a supporting plane to $v$ until it lies below the graph of $u$, and then lifting it up until it touches $u$, we obtain a supporting plane to $u$ at some point inside $E$.

up until it touches the graph of $u$ for the first time, we see that, for some constant $a \leq v(x)$,

$$
y \mapsto a+p \cdot(y-x)
$$

is a supporting plane to $u$ at some point $\bar{x} \in \bar{E}$; see Figure 2.1 .

Since $u=v$ on $\partial E$, we see that if $\bar{x} \in \partial E$, then $a=v(x)$ and thus $u(x)=v(x)$, and the plane is also supporting $u$ at $x \in E$. In conclusion, $p \in \partial u(E)$, proving the inclusion (2.4).

A first corollary is the celebrated Alexandrov maximum principle:

Theorem 2.6 (Alexandrov maximum principle). Let $u: \Omega \rightarrow \mathbb{R}$ be a convex function defined on an open, bounded, and convex domain $\Omega$. If $u=0$ on $\partial \Omega$, then

$$
|u(x)|^{n} \leq C_{n}(\operatorname{diam} \Omega)^{n-1} \operatorname{dist}(x, \partial \Omega)|\partial u(\Omega)| \quad \forall x \in \Omega,
$$

where $C_{n}$ is a geometric constant depending only on the dimension.

Proof. Let $(x, u(x))$ be a point on the graph of $u$, and let us consider the cone $C_{x}(y)$ with vertex on $(x, u(x))$ and base $\Omega$, that is, the graph of the one-homogeneous function which is 0 on $\partial \Omega$ and equal to $u(x)$ at $x$ (see Figure 2.2). Since by convexity $u(y) \leq C_{x}(y)$, Lemma 2.5 implies

$$
\left|\partial C_{x}(x)\right| \leq\left|\partial C_{x}(\Omega)\right| \leq|\partial u(\Omega)| .
$$

(Actually, as a consequence of the argument below, one sees that $\partial C_{x}(x)=\partial C_{x}(\Omega)$.) To conclude the proof, we have only to show that

$$
\left|\partial C_{x}(x)\right| \geq \frac{|u(x)|^{n}}{C_{n}(\operatorname{diam} \Omega)^{n-1} \operatorname{dist}(x, \partial \Omega)}
$$

for some dimensional constant $C_{n}>0$. Take $p$ with $|p|<|u(x)| / \operatorname{diam} \Omega$, and consider a plane with slope $p$. By first moving it down and lifting it up until it touches the graph of $C_{x}$, we see that it has to be supporting at some point $\bar{y} \in \Omega$. Since $C_{x}$ is a cone it also has to be supporting at $x$. This means

$$
\partial C_{x}(x) \supset B(0,|u(x)| / \operatorname{diam} \Omega) .
$$

Let now $\bar{x} \in \partial \Omega$ be such that $\operatorname{dist}(x, \partial \Omega)=|x-\bar{x}|$, and let $q$ be a vector with the same direction of $(\bar{x}-x)$ and with modulus less than $|u(x)| / \operatorname{dist}(x, \partial \Omega)$. Then the plane $u(x)+q \cdot(y-x)$ will be supporting $C_{x}$ at $x$ (see Figure 2.2), that is

$$
q:=\frac{\bar{x}-x}{|\bar{x}-x|} \frac{|u(x)|}{\mid \operatorname{dist}(x, \partial \Omega)} \in \partial C_{x}(x) .
$$



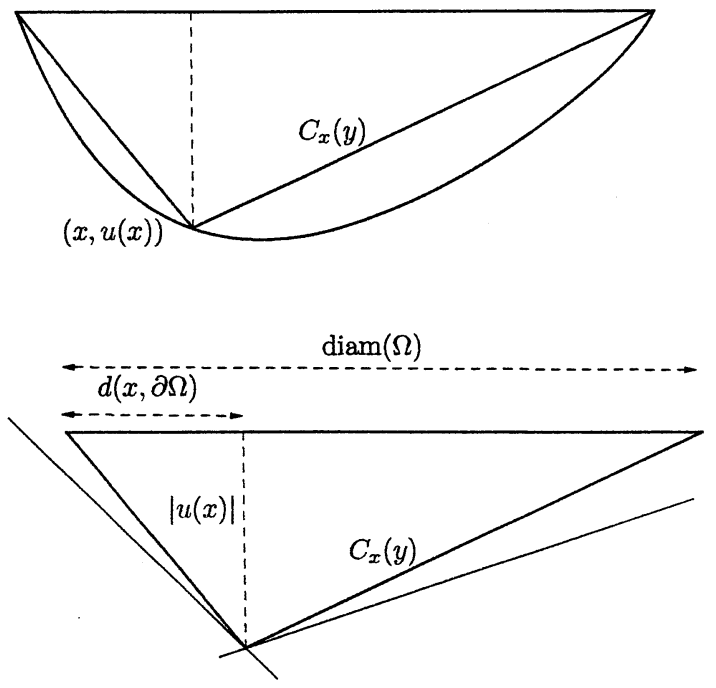

Figure 2.2. Every plane with slope $|p| \leq|u(x)| / \operatorname{diam}(\Omega)$ supports the graph of $C_{x}$ at $x$. Moreover, there exists a supporting plane whose slope has size comparable to $|u(x)| / \operatorname{dist}(x, \partial \Omega)$.

By convexity of $\partial C_{x}(x)$, we have that it contains the cone $\mathcal{C}$ generated by $q$ and $B(0,|u(x)| / \operatorname{diam} \Omega)$. Since

$$
|\mathcal{C}| \geq \frac{|u(x)|^{n}}{C_{n}(\operatorname{diam} \Omega)^{n-1} \operatorname{dist}(x, \partial \Omega)},
$$

this concludes the proof.

Another consequence of Lemma 2.5 is the following comparison principle.

Lemma 2.7. Let $u, v$ be convex functions defined on an open bounded convex set $\Omega$. If $u \geq v$ on $\partial \Omega$ and (in the sense of Monge-Ampère measures)

$$
\operatorname{det} D^{2} u \leq \operatorname{det} D^{2} v \quad \text { in } \Omega \text {, }
$$

then $u \geq v$ in $\Omega$.

Sketch of the proof. Up to replacing $v$ by $v+\varepsilon\left(\left|x-x_{0}\right|^{2}-\operatorname{diam}(\Omega)^{2}\right)$ where $x_{0}$ is an arbitrary point in $\Omega$ and then letting $\varepsilon \rightarrow 0$, we can assume that $\operatorname{det} D^{2} u<\operatorname{det} D^{2} v$.

The idea of the proof is simple: if $E:=\{u<v\}$ is nonempty, then we can apply Lemma 2.5 to obtain

$$
\int_{E} \operatorname{det} D^{2} u=\mu_{u}(E) \geq \mu_{v}(E)=\int_{E} \operatorname{det} D^{2} v .
$$

This is in contradiction with $\operatorname{det} D^{2} u<\operatorname{det} D^{2} v$ and concludes the proof.

An immediate corollary of the comparison principle is the uniqueness for Alexandrov solutions of the Dirichlet problem. We now actually state a stronger result concerning the stability of solutions, and we refer to [64, Lemma 5.3.1] for a proof. 
Theorem 2.8. Let $\Omega_{k} \subset \mathbb{R}^{n}$ be a family of convex domains, and let $u_{k}: \Omega_{k} \rightarrow \mathbb{R}$ be convex Alexandrov solutions of

$$
\begin{cases}\operatorname{det} D^{2} u_{k}=\mu_{k} & \text { in } \Omega_{k} \\ u_{k}=0 & \text { on } \partial \Omega_{k},\end{cases}
$$

where $\Omega_{k}$ converge to some convex domain $\Omega$ in the Hausdorff distance, and $\mu_{k}$ is a sequence of nonnegative Borel measures with $\sup _{k} \mu_{k}\left(\Omega_{k}\right)<\infty$ and which converge weakly* to a Borel measure $\mu$. Then $u_{k}$ converge uniformly to the Alexandrov solution of

$$
\begin{cases}\operatorname{det} D^{2} u=\mu & \text { in } \Omega \\ u=0 & \text { on } \partial \Omega .\end{cases}
$$

Thanks to the above stability result, we can now prove the existence of solutions by first approximating the right-hand side with atomic measures and then solving the latter problem via a Perron-type argument; see [64. Theorem 1.6.2] for more details.

Theorem 2.9. Let $\Omega$ be a bounded open convex domain, and let $\mu$ be a nonnegative Borel measure in $\Omega$. Then there exists an Alexandrov solution of

$$
\begin{cases}\operatorname{det} D^{2} u=\mu & \text { in } \Omega \\ u=0 & \text { on } \partial \Omega .\end{cases}
$$

Sketch of the proof. Let $\mu_{k}=\sum_{i=1}^{k} \alpha_{i} \delta_{x_{i}}, \alpha_{i} \geq 0$, be a family of atomic measures which converge weakly to $\mu$. By the stability result from Theorem 2.8 it suffices to construct a solution for $\mu_{k}$.

For this, we consider the family of all subsolution 2

$$
S\left[\mu_{k}\right]:=\left\{v: \Omega \rightarrow \mathbb{R}: v \text { convex, } v=0 \text { on } \partial \Omega, \operatorname{det} D^{2} v \geq \mu_{k}\right\} .
$$

First of all we notice that $S\left[\mu_{k}\right]$ is nonempty: indeed, it is not difficult to check that a function in this set is given by

$$
-A \sum_{i=1}^{k} C_{x_{i}}
$$

where $C_{x}$ is the "conical" one-homogeneous function which takes value -1 at $x$ and vanishes on $\partial \Omega$, and $A>0$ is a sufficiently large constant.

Then, by a variant of the argument used in the proof of Lemma 2.7, one shows that

$$
v_{1}, v_{2} \in S\left[\mu_{k}\right] \quad \Rightarrow \quad \max \left\{v_{1}, v_{2}\right\} \in S\left[\mu_{k}\right],
$$

and using Proposition 2.4 one sees that the set $S\left[\mu_{k}\right]$ is also closed under suprema. Hence the function $u_{k}:=\sup _{v \in S\left[\mu_{k}\right]} v$ belongs to $S\left[\mu_{k}\right]$, and one wants to show that $u_{k}$ is actually a solution (that is, it satisfies $\operatorname{det} D^{2} u_{k}=\mu_{k}$ ).

To prove this fact, one first shows that $\operatorname{det} D^{2} u_{k}$ is a measure concentrated on the set of points $\left\{x_{1}, \ldots, x_{k}\right\}$. Indeed, if not, there would be at least one point $\bar{x} \in \Omega \backslash\left\{x_{1}, \ldots, x_{k}\right\}$ and a vector $p \in \mathbb{R}^{n}$ such that $p \in \partial u(\bar{x}) \backslash \partial u\left(\left\{x_{1}, \ldots, x_{k}\right\}\right)$. This means that

$$
u_{k}\left(x_{j}\right)>u(\bar{x})+p \cdot\left(x_{j}-\bar{x}\right) \quad \forall j \in\{1, \ldots, k\},
$$

\footnotetext{
${ }^{2}$ The name subsolution is motivated by Lemma 2.7 Indeed, if $v \in S\left[\mu_{k}\right]$ and $u$ solves 2.5 with $\mu=\mu_{k}$ then $v \leq u$.
} 

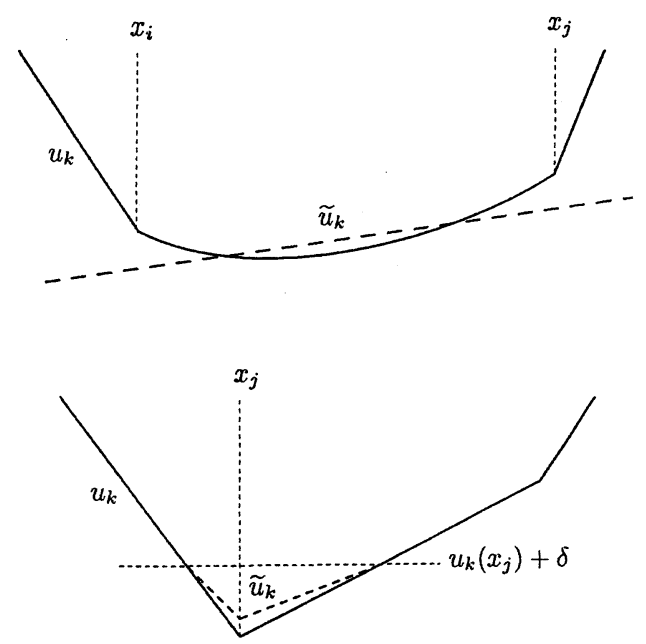

Figure 2.3. On the left the function $\widetilde{u}_{k}$ is obtained by cutting the graph of $u_{k}$ with a supporting hyperplane at some point $\bar{x} \in \Omega \backslash$ $\left\{x_{1}, \ldots, x_{k}\right\}$. On the right the function $\widetilde{u}_{k}$ is obtained by vertically dilating by a factor $(1-\delta)$ the graph of $u_{k}$ below the level $u_{k}\left(x_{j}\right)+\delta$.

hence we can define

$$
\widetilde{u}_{k}(x):=\max \left\{u_{k}(x), u_{k}(\bar{x})+p \cdot(y-\bar{x})+\delta\right\}
$$

for some $\delta>0$ sufficiently small to find a larger subsolution (see Figure 2.3), a contradiction.

Then one proves that $\operatorname{det} D^{2} u_{k}=\mu_{k}$. Indeed, if this was not the case, we would get that $\operatorname{det} D^{2} u_{k}=\sum_{i=1}^{k} \beta_{i} \delta_{x_{i}}$ with $\beta_{i} \geq \alpha_{i}$, and $\beta_{j}>\alpha_{j}$ for some $j \in\{1, \ldots, k\}$. Consider $p$ in the interior of $\partial u\left(x_{j}\right)$ (notice that $\partial u\left(x_{j}\right)$ is a convex set of volume $\beta_{j}>0$, hence it has nonempty interior), and assume without loss of generality that $p=0$ (otherwise simply subtract $p \cdot y$ from $u_{k}$ ). Then we define the function

$$
\widetilde{u}_{k}(x):= \begin{cases}u_{k}(x) & \text { if } u_{k}>u_{k}\left(x_{j}\right)+\delta \\ (1-\delta) u_{k}(x)+\delta\left[u_{k}\left(x_{j}\right)+\delta\right] & \text { if } u_{k} \leq u_{k}\left(x_{j}\right)+\delta\end{cases}
$$

for some $\delta>0$ sufficiently small (see Figure 2.3) and observe that this is a larger subsolution, again a contradiction.

Finally, the fact that $u_{k}=0$ on $\partial \Omega$ follows from the bound

$$
u_{k}(x) \geq-C \operatorname{dist}(x, \partial \Omega)^{1 / n}
$$

which is a consequence of Theorem 2.6.

2.2. The continuity method and existence of smooth solutions. Existence of smooth solutions to the Monge-Ampère equation dates back to the work of Pogorelov. These are obtained, together with nice and useful regularity estimates, through the well-celebrated method of continuity which now we briefly describe (see [62. Chapter 17] for a more detailed exposition). 
Let us assume that there exists a smooth convex solution $\bar{u}: \Omega \rightarrow \mathbb{R}$ td 3

$$
\begin{cases}\operatorname{det} D^{2} \bar{u}=\bar{f} & \text { in } \Omega \\ \bar{u}=0 & \text { on } \partial \Omega\end{cases}
$$

for some $\bar{f}>0$, and that we want to find a smooth solution td 4

$$
\begin{cases}\operatorname{det} D^{2} u=f & \text { in } \Omega \\ u=0 & \text { on } \partial \Omega .\end{cases}
$$

For this we define $f_{t}:=(1-t) \bar{f}+t f, t \in[0,1]$ and consider the one-parameter family of problems

$$
\begin{cases}\operatorname{det} D^{2} u_{t}=f_{t} & \text { in } \Omega \\ u_{t}=0 & \text { on } \partial \Omega .\end{cases}
$$

The method of continuity consists in showing that the set of $t \in[0,1]$ such that (2.7) is solvable is both open and closed, which implies the existence of a solution to our original problem. More precisely, let us assume that $f, \bar{f}$ are smooth, and consider the set

$$
\mathcal{C}:=\left\{u: \bar{\Omega} \rightarrow \mathbb{R} \text { convex functions of class } C^{2, \alpha}(\bar{\Omega}), u=0 \text { on } \partial \Omega\right\} .
$$

Consider the nonlinear map

$$
\begin{aligned}
\mathcal{F}: \mathcal{C} \times[0,1] & \longrightarrow C^{0, \alpha}(\bar{\Omega}) \\
(u, t) & \mapsto \operatorname{det} D^{2} u-f_{t} .
\end{aligned}
$$

We would like to show that

$$
\mathcal{T}:=\left\{t \in[0,1]: \text { there exists a } u_{t} \in \mathcal{C} \text { such that } \mathcal{F}\left(u_{t}, t\right)=0\right\}
$$

is both open and closed inside [0,1] (recall that, by assumption, $0 \in \mathcal{T}$ ). Openness follows from the Implicit Function Theorem in Banach spaces (see 62, Theorem 17.6]). Indeed, the Frechèt differential of $\mathcal{F}$ with respect to $u$ is given by the linearized Monge-Ampère operator (compare with Remark 2.1)

$$
D_{u} \mathcal{F}(u, t)[h]=\operatorname{det}\left(D^{2} u\right) u^{i j} h_{i j}, \quad h=0 \text { on } \partial \Omega,
$$

where we have set $h_{i j}:=\partial_{i j} h, u^{i j}$ is the inverse of $u_{i j}:=\partial_{i j} u$, and we are summing over repeated indices. Notice that if $u$ is bounded in $C^{2, \alpha}$ and $f$ is bounded from below by $\lambda$, then the smallest eigenvalue of $D^{2} u$ is bounded uniformly away from zero and the linearized operator becomes uniformly elliptic and with $C^{0, \alpha_{-}}$ coefficients (see also Remark 2.1). Therefore, classical Schauder's theory gives the invertibility of $D_{u} \mathcal{F}(u, t)$ [62, Chapter 6].

The task is now to prove closedness of $\mathcal{T}$. This is done through a priori estimates both at the interior and at the boundary. As we already noticed in Remark 2.1, the Monge-Ampère equation becomes uniformly elliptic on uniformly convex functions.

\footnotetext{
${ }^{3}$ Notice that if $\Omega$ is smooth and uniformly convex, it is always possible to find a couple $(\bar{u}, \bar{f})$ solving this equation. Indeed, one can take $\bar{u}$ to be any smooth uniformly convex function which coincides with $-\operatorname{dist}^{2}(\cdot, \partial \Omega)$ near the boundary of $\Omega$, and then set $\bar{f}:=\operatorname{det} D^{2} \bar{u}$.

${ }^{4}$ Here we are considering only the case in which $f=f(x)$, i.e., there is no dependence on the right-hand side from the "lower order" terms $u$ and $\nabla u$. The case $f=f(x, u, \nabla u)$ is just more tedious but the ideas/techniques are essentially the same. Note however that, in this case, one has to assume $\partial_{u} f \leq 0$ in order to apply the classical elliptic theory (in particular, the maximum principle) to the linearized operator; see for instance [62 Chapter 17].
} 
Since $\operatorname{det} D^{2} u$ is bounded away from zero, the main task is to establish an a priori bound on the $C^{2}$-norm of $u$ in $\bar{\Omega}$ since this will imply that the smallest eigenvalue of $D^{2} u$ is bounded away from zero. Once the equation becomes uniformly elliptic, the Evans-Krylov Theorem [62, Theorem 17.26’] will provide a priori $C^{2, \alpha}$-estimates up to the boundary, from which the closedness of $\mathcal{T}$ follows by the Ascoli-Arzelà Theorem.

Theorem 2.10. Let $\Omega$ be a uniformly convex domain 5 of class $C^{3}$, and let $u$ be a solution of (2.6) with $f \in C^{2}(\bar{\Omega})$ and $\lambda \leq f \leq 1 / \lambda$. Then there exists a constant $C$, depending only on $\Omega, \lambda,\|f\|_{C^{2}(\bar{\Omega})}$, such that

$$
\left\|D^{2} u\right\|_{C^{0}(\bar{\Omega})} \leq C \text {. }
$$

Notice that the uniform convexity of $\Omega$ is necessary to obtain regularity up to the boundary: indeed, if $D^{2} u$ is uniformly bounded, then (as we mentioned above) $u$ is uniformly convex on $\bar{\Omega}$, and hence by the Implicit Function Theorem $\partial \Omega=\{u=0\}$ is uniformly convex as well.

Theorem 2.10 together with an approximation procedure allows us to run the strategy described above to obtain the following existence result.

Theorem 2.11. Let $\Omega$ be a uniformly convex domain of class $C^{3}$. Then for all $f \in C^{2}(\bar{\Omega})$ with $\lambda \leq f \leq 1 / \lambda$ there exists a (unique) $C^{2, \alpha}(\bar{\Omega})$-solution to (2.6).

The proof of Theorem 2.10 is classical. However, since the ideas involved are at the basis of many other theorems in elliptic regularity, we give a sketch of the proof for the interested readers.

Sketch of the proof of Theorem 2.10. We begin by noticing that, because the linearized operator in (2.8) is degenerate elliptic (in the sense that, since we do not know yet that the eigenvalues of $D^{2} u$ are bounded away from zero and infinity, we cannot use any quantity involving its ellipticity constants), the maximum principle is essentially the only tool at our disposal.

Step 1 ( $C^{0}$ - and $C^{1}$-estimates). $C^{0}$ estimates can be obtained by a simple barrier construction. Indeed, it suffices to use Lemma 2.7 with $v(x):=\lambda^{-1 / n}\left(\left|x-x_{1}\right|^{2}-R^{2}\right)$ (where $x_{1}$ and $R$ are chosen so that $\Omega \subset B_{R}\left(x_{1}\right)$ ) to obtain a uniform lower bound on $u$.

To estimate the gradient, we note that by convexity

$$
\sup _{\Omega}|\nabla u|=\sup _{\partial \Omega}|\nabla u|
$$

so we need to estimate it only on the boundary. Since $u=0$ on $\partial \Omega$, any tangential derivative is zero, hence we only have to estimate the normal derivative. This can be done again with a simple barrier argument as above choosing, for any point $x_{0} \in \partial \Omega$,

where

$$
v_{ \pm}(x):=\lambda^{\mp 1 / n}\left(\left|x-x_{ \pm}\right|^{2}-R_{ \pm}^{2}\right),
$$

$$
x_{ \pm}:=x_{0}+R_{ \pm} \nu_{x_{0}}
$$

${ }^{5}$ We say that a domain is uniformly convex if there exists a radius $R$ such that

$$
\Omega \subset B_{R}\left(x_{0}+R \nu_{x_{0}}\right) \quad \text { for every } x_{0} \in \partial \Omega,
$$

where $\nu_{x_{0}}$ is the interior normal to $\Omega$ at $x_{0}$. Note that for a smooth domain this is equivalent to asking the second fundamental form of $\partial \Omega$ to be (uniformly) positive definite. 
and $0<R_{-}<R_{+}<\infty$ are chosen so that

$$
B_{R_{-}}\left(x_{-}\right) \subset \Omega \subset B_{R_{+}}\left(x_{+}\right) .
$$

In this way we get $v_{+} \leq u \leq v_{-}$, therefore

$$
-C \leq \partial_{\nu} u\left(x_{0}\right) \leq-\frac{1}{C}
$$

Step 2 ( $C^{2}$-estimates). This is the most delicate step. Given a unit vector $e$, we differentiate the equation

$$
\log \operatorname{det} D^{2} u=\log f
$$

once and two times in the direction of $e$ to get, respectively,

$$
L\left(u_{e}\right):=u^{i j}\left(u_{e}\right)_{i j}=(\log f)_{e}
$$

and

$$
u^{i j}\left(u_{e e}\right)_{i j}-u^{i l} u^{k j}\left(u_{e}\right)_{i j}\left(u_{e}\right)_{l k}=(\log f)_{e e}
$$

(recall that $u^{i j}$ denotes the inverse of $u_{i j}$ and that lower indices denote partial derivatives; see also Remark 2.1). By the convexity of $u, u^{i l} u^{k j}\left(u_{e}\right)_{i j}\left(u_{e}\right)_{l k} \geq 0$, hence

$$
L\left(u_{e e}\right) \geq(\log f)_{e e} \geq-C,
$$

for some constant $C$ depending only on $f$. Since $L(u)=u^{i j} u_{i j}=n$, we see that

$$
L\left(u_{e e}+M u\right) \geq 0
$$

for a suitable large constant $M$ depending on $f$. Hence, by the maximum principle,

$$
\sup _{\Omega}\left(u_{e e}+M u\right) \leq \sup _{\partial \Omega}\left(u_{e e}+M u\right) .
$$

Since $u$ is bounded, to get an estimate $D^{2} u$ we only have to estimate it on the boundary. Let us assume that $0 \in \partial \Omega$ and that locally

$$
\partial \Omega=\left\{\left(x_{1}, \ldots, x_{n}\right): x_{n}=\sum_{\alpha=1}^{n-1} \frac{\kappa_{\alpha}}{2} x_{\alpha}^{2}+O\left(|x|^{3}\right)\right\}
$$

for some constants $\kappa_{\alpha}>0$. Notice that, by the smoothness and uniform convexity of $\Omega$, we have $1 / C \leq \kappa_{\alpha} \leq C$. In this way

$$
u_{\alpha \alpha}(0)=-\kappa_{\alpha} u_{n}(0), \quad u_{\alpha \beta}(0)=0 \quad \forall \alpha \neq \beta \in\{1, \ldots, n-1\} .
$$

Thanks to (2.9) this gives

$$
\operatorname{Id}_{n-1} / C \leq\left(u_{\alpha \beta}(0)\right)_{\alpha, \beta \in\{1, \ldots, n-1\}} \leq C \operatorname{Id}_{n-1} .
$$

Noticing that

$$
f=\operatorname{det} D^{2} u=M^{n n}\left(D^{2} u\right) u_{n n}+\sum_{\alpha=1}^{n-1} M^{\alpha n}\left(D^{2} u\right) u_{\alpha n}
$$

with $M^{i j}\left(D^{2} u\right)$ the cofactor of $u_{i j}$, this identity and (2.14) will give an upper bound on $u_{n n}(0)$ once one has an upper bound on the mixed derivative $u_{\alpha n}(0)$ for $\alpha \in\{1, \ldots, n-1\}$. Hence, to conclude, we only have to provide an upper bound on $u_{\alpha n}(0)$. 
For this, let us consider the "rotational" derivative operator

$$
R_{\alpha n}=x_{\alpha} \partial_{n}-x_{n} \partial_{\alpha}, \quad \alpha \in\{1, \ldots, n-1\} .
$$

By the invariance of the determinant with respect to rotations (or by a direct computation), differentiating (2.10) we get

$$
L\left(R_{\alpha n} u\right)=u^{i j}\left(R_{\alpha n} u\right)_{i j}=R_{\alpha n}(\log f),
$$

hence, multiplying the above equation by $\kappa_{\alpha}$ and using (2.11), we get (recall that $\kappa_{\alpha}$ is a constant)

$$
\left|L\left(\left(1-\kappa_{\alpha} x_{n}\right) u_{\alpha}+\kappa_{\alpha} x_{\alpha} u_{n}\right)\right| \leq C .
$$

Since $u=0$ on $\partial \Omega$, thanks to (2.13), the uniform convexity of $\Omega$, and the bound on $|\nabla u|$, one easily computes that

$$
\left|\left(1-\kappa_{\alpha} x_{n}\right) u_{\alpha}+\kappa_{\alpha} x_{\alpha} u_{n}\right| \leq-A|x|^{2}+B x_{n} \quad \text { on } \partial \Omega
$$

for a suitable choice of constants $B \gg A \gg 1$ depending only on $\Omega$. Moreover,

$$
L\left(-A|x|^{2}+B x_{n}\right)=-A \sum_{i} u^{i i} \leq-\frac{n A}{\left(\operatorname{det} D^{2} u\right)^{1 / n}} \leq-\frac{n A}{\lambda^{1 / n}}
$$

where we have used the arithmetic-geometric mean inequality. Hence, choosing $A$ large enough, (2.15), (2.16), and (2.17), together with the comparison principle, imply

$$
\left|\left(1-\kappa_{\alpha} x_{n}\right) u_{\alpha}+\kappa_{\alpha} x_{\alpha} u_{n}\right| \leq-A|x|^{2}+B x_{n} \quad \text { in } \Omega .
$$

Dividing by $x_{n}$ and letting $x_{n} \rightarrow 0$, we finally get

$$
\left|u_{\alpha n}(0)\right| \leq C
$$

for a constant $C$ depending only on $\Omega$ and $f$, as desired.

2.3. Interior estimates and regularity of weak solutions. In the previous sections we have shown existence and uniqueness of weak solutions in general convex domains, and the existence of smooth solutions in smooth uniformly convex domains. To obtain smoothness of weak solutions in the interior of general convex domains, we need the following interior estimate, due to Pogorelov.

Theorem 2.12 (Pogorelov interior estimate). Let $u \in C^{4}(\Omega)$ be a solution of (2.6) with $f \in C^{2}(\Omega)$ and $\lambda \leq f \leq 1 / \lambda$. Then there exist a constant $C$, depending only on $\lambda$ and $\|f\|_{C^{2}}$, such that

$$
|u(x)| u_{11}(x) e^{\frac{\left(u_{1}(x)\right)^{2}}{2}} \leq C\left\|e^{u_{1}^{2} / 2}\left(1+\left|u_{1}\right|+|u|\right)\right\|_{L^{\infty}(\Omega)} \quad \text { for all } x \in \Omega .
$$

Sketch of the proof. Observing that (by convexity) $u \leq 0$ in $\Omega$, let us define

$$
w=(-u) u_{11} e^{\frac{\left(u_{1}\right)^{2}}{2}} \quad x \in \Omega .
$$

Let $x_{0}$ be a maximum point of $w$ in $\bar{\Omega}$, and notice that $x_{0} \in \Omega$ thanks to the boundary condition $u=0$. 
First we make a change of coordinate $x^{\prime}=A x$ with $\operatorname{det} A=1$, which leaves the direction $x_{1}$ invariant and such that $u_{i j}$ is diagonal at $x_{0}$ (see for instance [64, Chapter 4]). Then we compute

$$
\begin{aligned}
(\log w)_{i} & =\frac{u_{i}}{u}+\frac{u_{11 i}}{u_{11}}+u_{1} u_{1 i}, \\
(\log w)_{i j} & =\frac{u_{i j}}{u}-\frac{u_{i} u_{j}}{u^{2}}+\frac{u_{11 i j}}{u_{11}}-\frac{u_{11 i} u_{11 j}}{\left(u_{11}\right)^{2}}+u_{1} u_{1 i j}+u_{1 i} u_{1 j} .
\end{aligned}
$$

Since $x_{0}$ is a maximum point for $\log w$, we have

$$
0=(\log w)_{i}=\frac{u_{i}}{u}+\frac{u_{11 i}}{u_{11}}+u_{1} u_{1 i}
$$

and

$$
\begin{aligned}
0 \geq u^{i j}(\log w)_{i j}= & \frac{u^{i j} u_{i j}}{u}-\frac{u^{i j} u_{i} u_{j}}{u^{2}}+\frac{u^{i j} u_{11 i j}}{u_{11}}-\frac{u^{i j} u_{11 i} u_{11 j}}{\left(u_{11}\right)^{2}} \\
& +u_{1} u^{i j} u_{1 i j}+u^{i j} u_{1 i} u_{1 j} \\
= & \frac{L(u)}{u}-\frac{u^{i j} u_{i} u_{j}}{u^{2}}+\frac{L\left(u_{11}\right)}{u_{11}}-\frac{u^{i j} u_{11 i} u_{11 j}}{\left(u_{11}\right)^{2}} \\
& +u_{1} L\left(u_{1}\right)+u^{i j} u_{1 i} u_{1 j},
\end{aligned}
$$

where again $L(h)=u^{i j} h_{i j}$ and all functions are evaluated at $x_{0}$. Using (2.11) and (2.12) with $e=e_{1}$ and that $L(u)=n$, we get

$0 \geq \frac{n}{u}-\frac{u^{i j} u_{i} u_{j}}{u^{2}}+\frac{(\log f)_{11}}{u_{11}}+\frac{u^{i l} u^{k j} u_{1 i j} u_{1 k l}}{u_{11}}-\frac{u^{i j} u_{11 i} u_{11 j}}{\left(u_{11}\right)^{2}}+u_{1}(\log f)_{1}+u^{i j} u_{1 i} u_{1 j}$.

Now, recalling that $u_{i j}$ and $u^{i j}$ are diagonal at $x_{0}$, thanks to 2.20) we obtain

$$
\begin{aligned}
\frac{u^{i l} u^{k j} u_{1 i j} u_{1 k l}}{u_{11}} & -\frac{u^{i j} u_{11 i} u_{11 j}}{\left(u_{11}\right)^{2}}-\frac{u^{i j} u_{i} u_{j}}{u^{2}} \\
& =\frac{u^{i l} u^{k j} u_{1 i j} u_{1 k l}}{u_{11}}-\frac{u^{i j} u_{11 i} u_{11 j}}{\left(u_{11}\right)^{2}}-u^{i j}\left(\frac{u_{11 i}}{u_{11}}+u_{1} u_{1 i}\right)\left(\frac{u_{11 j}}{u_{11}}+u_{1} u_{1 j}\right) \\
& \geq-u^{11}\left(\frac{u_{111}}{u_{11}}+u_{1} u_{11}\right)^{2}=-\frac{\left(u_{1}\right)^{2}}{u^{2} u_{11}} .
\end{aligned}
$$

Plugging the above equation in (2.21) and taking again into account that $u_{i j}$ is diagonal, we get

$$
0 \geq \frac{n}{u}+\frac{(\log f)_{11}}{u_{11}}+u_{1}(\log f)_{1}-\frac{\left(u_{1}\right)^{2}}{u^{2} u_{11}}+u_{11} .
$$

Multiplying by $u^{2} u_{11} e^{\left(u_{1}\right)^{2}}$ and recalling the definition of $w$, we finally obtain

$$
0 \geq w^{2}-C w e^{\frac{\left(u_{1}\right)^{2}}{2}}\left(1+\left|u_{1}\right| u\right)-C e^{\left(u_{1}\right)^{2}}\left(u^{2}+\left(u_{1}\right)^{2}\right)
$$

for a suitable constant $C$ depending only on $f$, proving (2.19).

Combining the theorem above with the stability of weak solutions and the existence of smooth solutions, we obtain the following regularity result for strictly convex, solutions. Let us recall that a convex function $u$ is said to be strictly convex in $\Omega$ if, for any $x \in \Omega$ and $p \in \partial u(x)$,

$$
u(z)>u(x)+p \cdot(z-x) \quad \forall z \in \Omega \backslash\{x\},
$$

that is, any supporting plane to $u$ touches its graph at only one point. 
Theorem 2.13. Let $u: \Omega \rightarrow \mathbb{R}$ be a convex Alexandrov solution of (2.3) with $f \in C^{2}(\Omega)$ and $\lambda \leq f \leq 1 / \lambda$. Assume that $u$ is strictly convex inside $\Omega^{\prime} \subset \Omega$. Then $u \in C^{2}\left(\Omega^{\prime}\right)$.

Sketch of the proof. Fix $x_{0} \in \Omega^{\prime}, p \in \partial u\left(x_{0}\right)$, and consider the section of $u$ at height $t$ defined as

$$
S(x, p, t):=\{y \in \Omega: u(y) \leq u(x)+p \cdot(y-x)+t\} .
$$

Since $u$ is strictly convex, we can choose $t>0$ small enough so that $S\left(x_{0}, p, t\right) \Subset \Omega^{\prime}$. Then we consider $S_{\varepsilon}$ a sequence of smooth uniformly convex sets converging to $S\left(x_{0}, p, t\right)$ and apply Theorem 2.11 to find a function $v_{\epsilon} \in C^{2, \alpha}\left(S_{\varepsilon}\right)$ solving

$$
\begin{cases}\operatorname{det} D^{2} v_{\epsilon}=f * \rho_{\epsilon} & \text { in } S_{\varepsilon} \\ v_{\epsilon}=0 & \text { on } \partial S_{\varepsilon} .\end{cases}
$$

By Schauder's theory $v_{\epsilon}$ are of class $C^{\infty}$ inside $S_{\varepsilon}$, so we can apply Theorem 2.12 to deduce that

$$
\left|D^{2} v_{\varepsilon}\right| \leq C \quad \text { in } S\left(x_{0}, p, t / 2\right)
$$

for $\varepsilon$ sufficiently small. Since $S_{\varepsilon} \rightarrow S\left(x_{0}, p, t\right)$ and $u(x)=u\left(x_{0}\right)+p \cdot x+t$ on $\partial S\left(x_{0}, p, t\right)$, by uniqueness of weak solutions we deduce that $v_{\varepsilon}+u\left(x_{0}\right)+p \cdot x+t \rightarrow u$ uniformly as $\varepsilon \rightarrow 0$, hence $\left|D^{2} u\right| \leq C$ in $S\left(x_{0}, p, t / 2\right)$. This makes the equation uniformly elliptic, so $u \in C^{2}\left(S\left(x_{0}, p, t / 4\right)\right)$. By the arbitrariness of $x_{0}$ we obtain that $u \in C^{2}\left(\Omega^{\prime}\right)$, as desired.

2.4. Further regularity results for weak solutions. Up to now, we have shown regularity for weak solutions when the right-hand side is at least $C^{2}$. We now investigate their regularity under weaker smoothness assumptions on the righthand side. As we shall see later, this question, apart from its own interest, also has several applications.

In the 1990s Caffarelli developed a regularity theory for Alexandrov solutions, showing that strictly convex solutions of (1.1) are locally $C^{1, \alpha}$ provided $0<\lambda \leq$ $f \leq 1 / \lambda$ for some $\lambda \in \mathbb{R}$; see [16, 18, 19].

We emphasize that, for weak solutions, strict convexity is not implied by the positivity of $f$ and it is actually necessary for regularity; see Remark 2.19 below.

Theorem 2.14 (Caffarelli). Let $u: \Omega \rightarrow \mathbb{R}$ be a strictly convex solution of (2.3) with $\lambda \leq f \leq 1 / \lambda$. Then $u \in C_{\mathrm{loc}}^{1, \alpha}(\Omega)$ for some universal $\alpha$. More precisely, for every $\Omega^{\prime} \Subset \Omega$, there exists a constant $C$, depending on $\lambda, \Omega^{\prime}$, and the modulus of strict convexity of $u$, such that

$$
\sup _{\substack{x, y \in \Omega^{\prime} \\ x \neq y}} \frac{|\nabla u(x)-\nabla u(y)|}{|x-y|^{\alpha}} \leq C .
$$

To explain the ideas behind the proof of the above theorem, let us point out the following simple properties of solutions to the Monge-Ampère equation (which are just another manifestation of its degenerate ellipticity): if $A$ is a linear transformation with $\operatorname{det} A=1$ and $u$ is a solution of the Monge-Ampère equation with right-hand side $f$, then $u \circ A$ is a solution to the Monge-Ampère equation with right-hand side $f \circ A$. This affine invariance creates serious obstructions to obtain a local regularity theory. Indeed, for instance, the functions

$$
u_{\varepsilon}\left(x_{1}, x_{2}\right)=\frac{\varepsilon x_{1}^{2}}{2}+\frac{x_{2}^{2}}{2 \varepsilon}-1
$$


are solutions to $\operatorname{det} D^{2} u_{\varepsilon}=1$ on $\left\{u_{\varepsilon} \leq 0\right\}$. Thus, unless the level set $\left\{u_{\varepsilon}=0\right\}$ is sufficiently "round", there is no hope to obtain a priori estimates on $u$. The intuition of Caffarelli was to use the so-called John's Lemma 69]:

Lemma 2.15. Let $\mathcal{K} \subset \mathbb{R}^{n}$ be a bounded convex set with non-empty interior. Then there exists a unique ellipsoid $E$ of maximal volume contained in $\mathcal{K}$. Moreover this ellipsoid satisfies

$$
E \subset \mathcal{K} \subset n E,
$$

where $n E$ denotes the dilation of $E$ by a factor $n$ with respect to its center.

In particular, if we define a convex set $\Omega$ to be normalized if

$$
B_{1} \subset \Omega \subset n B_{1},
$$

then Lemma 2.15 says that, for every bounded open convex set $\Omega$, there is an affine transformation $A$ such that $A(\Omega)$ is normalized. Note that if $u$ solves

$$
\lambda \leq \operatorname{det} D^{2} u \leq 1 / \lambda \quad \text { in } \Omega, \quad u=0 \quad \text { on } \partial \Omega,
$$

and $A$ normalizes $\Omega$, then $v:=(\operatorname{det} A)^{2 / n} u \circ A^{-1}$ solves

$$
\lambda \leq \operatorname{det} D^{2} v \leq 1 / \lambda \quad \text { in } A(\Omega), \quad u=0 \quad \text { on } \partial(A(\Omega)) .
$$

Thanks to the above discussion and a suitable localization argument based on the strict convexity of $u$ as in the proof of Theorem 2.13. Theorem 2.14 is a consequence of the following:

Theorem 2.16. Let $\Omega$ be a normalized convex set, and let $u$ be a solution of (2.6) with $\lambda \leq f \leq 1 / \lambda$. Then there exist positive constants $\alpha=\alpha(n, \lambda)$ and $C=C(n, \lambda)$ such that

$$
\|u\|_{C^{1, \alpha}\left(B_{1 / 2}\right)} \leq C .
$$

In the proof of the above theorem, a key step consists in showing that solutions of (2.6) on normalized domains have a universal modulus of strict convexity. A fundamental ingredient to prove this fact is the following important result of Caffarelli [16]:

Proposition 2.17. Let $u$ be a solution of

$$
\lambda \leq \operatorname{det} D^{2} u \leq 1 / \lambda
$$

inside a convex set $\Omega$, and let $\ell: \mathbb{R}^{n} \rightarrow \mathbb{R}$ be a linear function supporting $u$ at some point $\bar{x} \in \Omega$. If the convex set

$$
W:=\{x \in \Omega: u(x)=\ell(x)\}
$$

contains more than one point, then it cannot have extremal points in $\Omega$.

This statement says that if a solution coincides with one of its supporting planes on more than one point (that is, it is not strictly convex), then the contact set has to cross the domain. Hence, if the boundary conditions are such that $u$ cannot coincide with an affine function along a segment crossing $\Omega$, one deduces that $u$ must be strictly convex. In particular by noticing that when $\Omega$ is normalized, the class of solutions is compact with respect to the uniform convergence (this is a consequence of Proposition 2.4 and the fact that the family of normalized convex domains is a compact), a simple contradiction argument based on compactness shows the modulus of strict convexity must be universal. From this, one deduces the following estimate: 


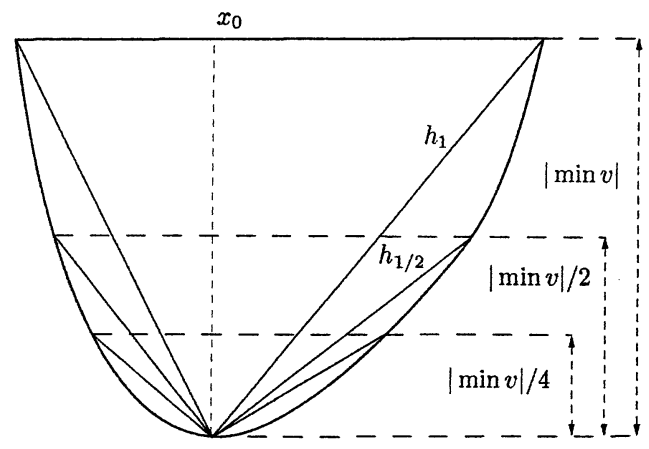

FigURE 2.4. The function $v$ looks flatter and flatter near its minimum $x_{0}$.

Lemma 2.18. Let $\Omega$ be a normalized convex domain, and let $v$ be a solution of (2.6) with $\lambda \leq f \leq 1 / \lambda$. Let $x_{0}$ be a minimum point for $u$ in $\Omega$, and for any $\beta \in(0,1]$ denote by $C_{\beta} \subset \mathbb{R}^{n+1}$ the cone with vertex $\left(x_{0}, v\left(x_{0}\right)\right)$ and base $\{v=(1-\beta) \min v\} \times\{(1-\beta) \min v\}$. Then, if $h_{\beta}$ is the function whose graph is given by $C_{\beta}$ (see Figure 2.4), there exists a universal constant $\delta_{0}>0$ such that

$$
h_{1 / 2} \leq\left(1-\delta_{0}\right) h_{1} .
$$

Now the proof of Theorem 2.16 goes as follows. For any $k \in \mathbb{N}$, we can consider the convex set $\Omega_{k}:=\left\{u \leq\left(1-2^{-k}\right) \min u\right\}$. Then, if we renormalize $\Omega_{k}$ through an affine map $A_{k}$, we can apply Lemma 2.18 to the function $v=\left(\operatorname{det} A_{k}\right)^{2 / n} u \circ A_{k}($ see (2.24) ) and transfer the information back to $u$ to deduce that $h_{2^{-(k+1)}} \leq\left(1-\delta_{0}\right) h_{2^{-k}}$. Therefore, by iterating this estimate, we obtain

$$
h_{2^{-k}} \leq\left(1-\delta_{0}\right)^{k} h_{1} \quad \forall k \in \mathbb{N} .
$$

From this fact it follows that $v$ is $C^{1, \alpha}$ at the minimum, in the sense that

$$
u(y)-u\left(x_{0}\right) \leq C\left|y-x_{0}\right|^{1+\alpha} ;
$$

see Figure 2.4.

Now, given any point $x \in \Omega^{\prime} \Subset \Omega$ and $p \in \partial u(x)$, we can repeat the same argument with the function $u(y)-p \cdot(y-x)$ in place of $u$, replacing $\Omega$ with the section $S(x, p, t)$ (see (2.22) $)$ for some $t$ small but fixed chosen such that $S(x, p, t) \Subset \Omega 6$ Then the above estimate gives

$$
u(y)-u(x)-p \cdot(y-x) \leq C|y-x|^{1+\alpha} \quad \forall p \in \partial u(x) .
$$

By the arbitrariness of $x \in \Omega^{\prime}$, it is well known that the above estimate implies the desired $C_{\text {loc }}^{1, \alpha}$ regularity of $u$ (see for instance [38, Lemma 3.1]).

Let us notice that a direct proof of Theorem 2.16 (which avoids any compactness argument) has been given by Forzani and Maldonado [59], allowing one to compute the explicit dependence of $\alpha$ on $\lambda$.

Remark 2.19 (On the strict convexity of weak solutions). As we mentioned before, strict convexity is not just a technical assumption but it is necessary to obtain regularity. Indeed, as also mentioned at the beginning of Section 2, there are

\footnotetext{
${ }^{6}$ To be more precise, one needs to apply an affine transformation so that the section $S(x, p, t)$ becomes a normalized convex set.
} 
Alexandrov solutions to the Monge-Ampère equation with smooth positive righthand side which are not $C^{2}$. For instance, the function

$$
u\left(x_{1}, x^{\prime}\right):=\left|x^{\prime}\right|^{2-2 / n}\left(1+x_{1}^{2}\right), \quad n \geq 3,
$$

is $C^{1,1-2 / n}$ and solves $\operatorname{det} D^{2} u=c_{n}\left(1+x_{1}^{2}\right)^{n-2}\left(1-x_{1}^{2}\right)>0$ inside $B_{1 / 2}$. Furthermore, having only the bound $\lambda \leq \operatorname{det} D^{2} u \leq 1 / \lambda$ is not even enough for $C^{1}$ regularity: the function

$$
u\left(x_{1}, x^{\prime}\right):=\left|x^{\prime}\right|+\left|x^{\prime}\right|^{n / 2}\left(1+x_{1}^{2}\right), \quad n \geq 3,
$$

is merely Lipschitz and solves $\lambda \leq \operatorname{det} D^{2} u \leq 1 / \lambda$ in a small convex neighborhood of the origin.

Alexandrov showed in [3] that, in contrast with the above counterexamples, in two dimensions every solution of $\operatorname{det} D^{2} u \geq \lambda$ is strictly convex (see also 21]). Recently, in 93, Mooney established that, in every dimension, the set of points where an Alexandrov solution of $\operatorname{det} D^{2} u \geq \lambda$ coincides with one of its supporting planes has vanishing $\mathcal{H}^{n-1}$ measure.

In the case when $f$ is Hölder continuous, Caffarelli proved that $u$ is locally $C^{2, \alpha}$ [17, improving Theorem 2.13

Theorem 2.20. Let $\Omega$ be a normalized convex set, and let $u$ be an Alexandrov solution of (2.6) with $\lambda \leq f \leq 1 / \lambda$ and $f \in C^{0, \alpha}(\Omega)$. Then $\|u\|_{C^{2, \alpha}\left(B_{1 / 2}\right)} \leq C$ for some constant $C$ depending only on $n, \lambda$, and $\|f\|_{C^{0, \alpha}\left(B_{1}\right)}$.

The proof of the above theorem is based on showing that, under the assumption that $f$ is almost a constant (say very close to 1 ), $u$ is very close to the solution of (2.6) with right-hand side 1 . Since this latter function has interior a priori estimates (by Theorem 2.12), an approximation/interpolation argument permits us to show that the $C^{2}$-norm of $u$ remains bounded. With this line of reasoning, one can also prove the following theorem of Caffarelli [17]:

Theorem 2.21. Let $\Omega$ be a normalized convex set, and let $u$ be a solution of (2.6). Then, for every $p>1$, there exist positive constants $\delta(p)$ and $C=C(p)$ such that if $\|f-1\|_{\infty} \leq \delta(p)$, then $\|u\|_{W^{2, p}\left(B_{1 / 2}\right)} \leq C$.

We notice that by localizing the above result on small sections, one deduces that if $u$ is a strictly convex solutions of (3.4) with $f$ continuous, then $u \in W_{\text {loc }}^{2, p}$ for all $p<\infty$ (see [17] or [64, Chapter 6]).

Wang [111] showed that for any $p>1$ there exists a function $f$ satisfying $0<$ $\lambda(p) \leq f \leq 1 / \lambda(p)$ such that $u \notin W_{\text {loc }}^{2, p}$. This counterexample shows that the results of Caffarelli are more or less optimal. However, an important question which remained open for a long time was whether solutions of (3.4) with $0<\lambda \leq f \leq 1 / \lambda$ could be at least $W_{\text {loc }}^{2,1}$, or even $W_{\text {loc }}^{2,1+\varepsilon}$ for some $\varepsilon=\varepsilon(n, \lambda)>0$. The question of $W_{\text {loc }}^{2,1}$-regularity has been recently solved by the authors in 35. Following the ideas introduced there, later in [40,100] the result has been refined to $u \in W_{\text {loc }}^{2,1+\varepsilon}$ for some $\varepsilon>0$.

Theorem 2.22. Let $\Omega$ be a normalized convex set, and let $u$ be be an Alexandrov solution of (2.6) with $\lambda \leq f \leq 1 / \lambda$. Then there exist positive constants $\varepsilon=\varepsilon(n, \lambda)$ and $C=C(n, \lambda)$ such that $\|u\|_{W^{2,1+\varepsilon}\left(B_{1 / 2}\right)} \leq C$. 
Note that all the previous results hold for strictly convex solutions of (3.4). Indeed, it suffices to pick a section $S(x, p, t) \Subset \Omega$, choose an affine transformation $A$ so that $A(S(x, p, t))$ is normalized (see (2.24) $)$, and then apply the results above with $A(S(x, p, t))$ in place of $\Omega$.

We now briefly sketch the ideas behind the proof of the $W^{2,1}$-regularity in 35 . To prove that the distributional Hessian $D_{\mathcal{D}}^{2} u$ of an Alexandrov solution is an $L^{1}$ function, it is enough to prove an a priori equi-integrability estimate on smooth solutions 7 To do this, a key observation is that any domain $\Omega$ endowed with the Lebesgue measure and the family of "balls" given by the sections $\{S(x, p, t)\}_{x \in \Omega, t \in \mathbb{R}}$ of solutions of (2.6) as defined in (2.22) is a space homogenous type in the sense of Coifman and Weiss; see [1,24, 65]. In particular Stein's Theorem implies that if

$$
\mathcal{M}\left(D^{2} u\right)(x):=\sup _{t} f_{S(x, p, t)}\left|D^{2} u\right| \in L^{1},
$$

then $\left|D^{2} u\right| \in L \log L$, that is $\int\left|D^{2} u\right| \log \left(2+\left|D^{2} u\right|\right) \leq C$. Hence one wants to prove that $\mathcal{M}\left(D^{2} u\right) \in L^{1}$, and the key estimate in 35] consists in showing that

$$
\left\|\mathcal{M}\left(D^{2} u\right)\right\|_{L^{1}} \leq C\left\|D^{2} u\right\|_{L^{1}}
$$

for some constant $C=C(n, \lambda)$, which proves the result.

A more careful application of these ideas then gives a priori $W^{2,1+\varepsilon}$-estimates. As shown for instance in [50], the approach in [40] can be also used to give a very direct and short proof of the $W^{2, p}$-estimates of Caffarelli in 17.

Using Theorem 2.22 the authors could show in 36 the following stability property of solutions of (2.3) in the strong Sobolev topology. Due the highly nonlinear character of the Monge-Ampère equation, the result is nontrivial.

Theorem 2.23. Let $\Omega_{k} \subset \mathbb{R}^{n}$ be a family of bounded convex domains, and let $u_{k}: \Omega_{k} \rightarrow \mathbb{R}$ be convex Alexandrov solutions of

$$
\begin{cases}\operatorname{det} D^{2} u_{k}=f_{k} & \text { in } \Omega_{k} \\ u_{k}=0 & \text { on } \partial \Omega_{k}\end{cases}
$$

with $0<\lambda \leq f_{k} \leq 1 / \lambda$. Assume that $\Omega_{k}$ converges to some bounded convex domain $\Omega$ in the Hausdorff distance, and $f_{k} \mathbf{1}_{\Omega_{k}}$ converges to $f$ in $L_{\text {loc }}^{1}(\Omega)$. Then, if $u$ denotes the unique Alexandrov solution of

$$
\begin{cases}\operatorname{det} D^{2} u=f & \text { in } \Omega \\ u=0 & \text { on } \partial \Omega\end{cases}
$$

for any $\Omega^{\prime} \Subset \Omega$ we have

$$
\left\|u_{k}-u\right\|_{W^{2,1}\left(\Omega^{\prime}\right)} \rightarrow 0 \quad \text { as } k \rightarrow \infty .
$$

Let us conclude this section by mentioning that Savin recently introduced new techniques to obtain global versions of all the above regularity results under suitable regularity assumptions on the boundary 97 99.

\footnotetext{
${ }^{7}$ Note that it is pretty simple to show that for smooth convex functions $\left\|D^{2} u\right\|_{L^{1}} \leq C$ osc $u$. However, since an equi-bounded sequence of $L^{1}$-functions can converge to a singular measure, this is not enough to prevent concentration of the Hessians in the limit, and this is why we need an equi-integrability estimate on $D^{2} u$ which is independent of $u$.
} 


\subsection{An application: global existence for the semigeostrophic equations.}

Let us conclude this discussion on the regularity of weak solutions by showing an application of Theorem 2.22 proving the existence of distributional solutions for the semigeostrophic system.

The semigeostrophic equations are a simple model used in meteorology to describe large-scale atmospheric flows. As explained for instance in [13, Section 2.2] (see also 33 for a more complete exposition), these equations can be derived from the 3-d Euler equations, with Boussinesq and hydrostatic approximations, subject to a strong Coriolis force. Since for large-scale atmospheric flows the Coriolis force dominates the advection term, the flow is mostly bidimensional. For this reason, the study of the semigeostrophic equations in 2-d or 3-d is pretty similar, and in order to simplify our presentation we focus here on the 2-dimensional periodic case.

The semigeostrophic system can be written as

$$
\left\{\begin{array}{l}
\partial_{t} \nabla p_{t}+\left(\boldsymbol{u}_{t} \cdot \nabla\right) \nabla p_{t}+\nabla^{\perp} p_{t}+\boldsymbol{u}_{t}=0 \\
\nabla \cdot \boldsymbol{u}_{t}=0 \\
p_{0}=\bar{p}
\end{array}\right.
$$

where $\boldsymbol{u}_{t}: \mathbb{R}^{2} \rightarrow \mathbb{R}^{2}$ and $p_{t}: \mathbb{R}^{2} \rightarrow \mathbb{R}$ are periodic functions corresponding respectively to the velocity and the pressure, and $\nabla^{\perp} p_{t}$ is the $\pi / 2$ counterclockwise rotation of $\nabla p$.

As shown in 33 , energetic considerations show that it is natural to assume that $p_{t}$ is $(-1)$-convex, i.e., the function $P_{t}(x):=p_{t}(x)+|x|^{2} / 2$ is convex on $\mathbb{R}^{2}$. If we denote with $\mathcal{L}_{\mathbb{T}^{2}}$ the Lebesgue measure on the 2-dimensional torus, then formally $\rho_{t}:=\left(\nabla P_{t}\right)_{\sharp} \mathcal{L}_{\mathbb{T}^{2}}$ satisfies the following dual problem (see for instance [7, Appendix]):

$$
\left\{\begin{array}{l}
\partial_{t} \rho_{t}+\nabla \cdot\left(\mathcal{U}_{t} \rho_{t}\right)=0 \\
\mathcal{U}_{t}(x)=\left(x-\nabla P_{t}^{*}(x)\right)^{\perp} \\
\rho_{t}=\left(\nabla P_{t}\right)_{\sharp} \mathcal{L}_{\mathbb{T}^{2}} \\
P_{0}(x)=\bar{p}(x)+|x|^{2} / 2,
\end{array}\right.
$$

where $P_{t}^{*}$ is the convex conjugate of $P_{t}$, namely

$$
P_{t}^{*}(y):=\sup _{x \in \mathbb{R}^{2}}\left\{y \cdot x-P_{t}(x)\right\} .
$$

The dual problem (2.28) is nowadays pretty well understood. In particular, Benamou and Brenier proved in [13] existence of weak solutions to (2.28). On the contrary, much less is known about the original system (2.27). Formally, given a solution $\left(\rho_{t}, P_{t}\right)$ of (2.28), the pair $\left(p_{t}, \boldsymbol{u}_{t}\right)$ given by

$$
\left\{\begin{array}{l}
p_{t}(x):=P_{t}(x)-|x|^{2} / 2 \\
\boldsymbol{u}_{t}(x):=\partial_{t} \nabla P_{t}^{*}\left(\nabla P_{t}(x)\right)+D^{2} P_{t}^{*}\left(\nabla P_{t}(x)\right)\left(\nabla P_{t}(x)-x\right)^{\perp}
\end{array}\right.
$$

solves 2.27).

Since a priori $P_{t}^{*}$ is just a convex function without any smoothness, $D^{2} P_{t}^{*}$ is a matrix-valued measure, thus it is not clear what is the meaning of giving $D^{2} P_{t}^{*}\left(\nabla P_{t}(x)\right)$ in the previous formula. However, since $\rho_{t}$ solves a continuity equation with a divergence-free vector field (notice that $\mathcal{U}_{t}$ is the rotated gradient of

\footnotetext{
${ }^{8}$ See (3.1) for the definition of $\left(\nabla P_{t}\right)_{\sharp} \mathcal{L}_{\mathbb{T}^{2}}$.
} 
the function $|x|^{2} / 2-P_{t}^{*}(x)$, see (2.28) $)$, the only available bound on $\rho_{t}$ is

$$
0<\lambda \leq \rho_{t} \leq 1 / \lambda \quad \forall t>0
$$

provided this bound holds at $t=0$.

In addition, the relation $\rho_{t}=\left(\nabla P_{t}\right)_{\sharp} \mathcal{L}_{\mathbb{T}^{2}}$ implies that $\left(\nabla P_{t}^{*}\right)_{\sharp} \rho_{t}=\mathcal{L}_{\mathbb{T}^{2}}$ (since $\nabla P_{t}^{*}$ is the inverse of $\left.\nabla P_{t}\right)$, and one can show that $P_{t}^{*}$ solves in the Alexandrov sense the Monge-Ampère equation

$$
\operatorname{det}\left(D^{2} P_{t}^{*}\right)=\rho_{t}
$$

(see 7 for more details). Hence, thanks to (2.30), one can apply Theorem 2.22 to deduce that $D^{2} P_{t}^{*} \in L^{1+\varepsilon}$, which allows us to give a meaning to the velocity field $\boldsymbol{u}_{t}$ defined in (2.29), and to prove that $\left(p_{t}, \boldsymbol{u}_{t}\right)$ solves (2.27).

This has been done recently in [7, where the following result has been proved (see 8 for an extension of this result to three dimensions):

Theorem 2.24. Let $\bar{p}: \mathbb{R}^{2} \rightarrow \mathbb{R}$ be a $\mathbb{Z}^{2}$-periodic function such that $\bar{p}(x)+|x|^{2} / 2$ is convex, and assume that the measure $(\mathrm{Id}+\nabla \bar{p})_{\sharp} \mathcal{L}_{\mathbb{T}^{2}}$ is absolutely continuous with respect to the Lebesgue measure with density $\bar{\rho}$, namely

$$
(\mathrm{Id}+\nabla \bar{p})_{\sharp} \mathcal{L}_{\mathbb{T}^{2}}=\bar{\rho} .
$$

Moreover, let us assume $\lambda \leq \bar{\rho} \leq 1 / \lambda$ for some $\lambda>0$.

Let $\rho_{t}$ be a solution of (2.28) starting from $\bar{\rho}$, and let $P_{t}: \mathbb{R}^{2} \rightarrow \mathbb{R}$ be the (unique up to an additive constant) convex function such that $\left(\nabla P_{t}\right)_{\sharp} \mathcal{L}_{\mathbb{T}^{2}}=\rho_{t}$ and $P_{t}(x)-|x|^{2} / 2$ is $\mathbb{Z}^{2}$-periodic 9 Denote by $P_{t}^{*}: \mathbb{R}^{2} \rightarrow \mathbb{R}$ its convex conjugate. Then the couple $\left(p_{t}, \boldsymbol{u}_{t}\right)$ defined in (2.29) is a distributional solution of (2.27).

Let us point out that (2.30) is essentially the only regularity property for solutions of (2.28) that is stable in time. Indeed, even if we start from a smooth initial datum $\bar{\rho}$, due to the nonlinear coupling between the density $\varrho_{t}$ and the velocity field $\mathcal{U}_{t}$, any "simple" higher order estimate blows up in finite time (it is however possible to prove short time existence for smooth solutions, see [83]). In view of these considerations, it is clear that understanding the regularity of solutions of the Monge-Ampère equation with its right-hand side just bounded away from zero and infinity is a key step for the proof of Theorem 2.24,

\section{The OPTIMAL TRANSPORT PROBLEM}

The Monge transportation problem is more than 200 years old 91, and it has generated in the last years a huge amount of work.

Originally Monge wanted to move, in the Euclidean space $\mathbb{R}^{3}$, a rubble (déblais) to build up a mound or fortification (remblais) minimizing the transportation cost. In Monge's original formulation, the cost to transport some mass $m$ from $x$ to $y$ was given by $m|x-y|$. However, it makes sense to consider more general cost functions $c$, where $c(x, y)$ represents the cost to move a unit mass from $x$ to $y$.

Hence, nowadays, the optimal transport problem is formulated in the following general form: given two probability measures $\mu$ and $\nu$ (representing the rubble and the mound, respectively) defined on the measurable spaces $X$ and $Y$, find a measurable map $T: X \rightarrow Y$ with $T_{\sharp} \mu=\nu$, i.e.,

$$
\nu(A)=\mu\left(T^{-1}(A)\right) \quad \forall A \subset Y \text { measurable, }
$$

\footnotetext{
${ }^{9}$ The existence of such a map is a consequence of Theorem 3.7 see 7 , Theorem 2.1].
} 
in such a way that $T$ minimizes the transportation cost. This means

$$
\int_{X} c(x, T(x)) d \mu(x)=\min _{S_{\#} \mu \nu}\left\{\int_{X} c(x, S(x)) d \mu(x)\right\},
$$

where $c: X \times Y \rightarrow \mathbb{R}$ is some given cost function, and the minimum is taken over all measurable maps $S: X \rightarrow Y$ such that $S_{\#} \mu=\nu$. When the transport condition $T_{\#} \mu=\nu$ is satisfied, we say that $T$ is a transport map, and if $T$ also minimizes the cost we call it an optimal transport map.

Even in Euclidean spaces with the cost $c$ equal to the Euclidean distance or its square, the problem of the existence of an optimal transport map is far from being trivial. Moreover, it is easy to build examples where the Monge problem is ill posed simply because there is no transport map: this happens for instance when $\mu$ is a Dirac mass while $\nu$ is not. This means that one needs some restrictions on the measures $\mu$ and $\nu$.

We notice that, if $X, Y \subset \mathbb{R}^{n}, \mu(d x)=f(x) d x$, and $\nu(d y)=g(y) d y$, if $S: X \rightarrow Y$ is a sufficiently smooth transport map, one can rewrite the transport condition $S_{\#} \mu=\nu$ as a Jacobian equation. Indeed, if $\chi: \mathbb{R}^{n} \rightarrow \mathbb{R}$ denotes a test function, the condition $S_{\#} \mu=\nu$ gives

$$
\int_{\mathbb{R}^{n}} \chi(S(x)) f(x) d x=\int_{\mathbb{R}^{n}} \chi(y) g(y) d y .
$$

Now, assuming in addition that $S$ is a diffeomorphism, we can set $y=S(x)$ and use the change of variable formula to obtain that the second integral is equal to

$$
\int_{\mathbb{R}^{n}} \chi(S(x)) g(S(x))|\operatorname{det}(\nabla S(x))| d x .
$$

By the arbitrariness of $\chi$, this gives the Jacobian equation

$$
f(x)=g(S(x))|\operatorname{det}(\nabla S(x))| \quad \text { a.e. },
$$

as desired.

3.1. The quadratic cost on $\mathbb{R}^{n}$. In 14,15 , Brenier considered the cases $X=$ $Y=\mathbb{R}^{n}$ and $c(x, y)=|x-y|^{2} / 2$, and proved the following theorem (which was also obtained independently by Cuesta-Albertos and Matrán [31] and by Rachev and Rüschendorf [96]). For an idea of the proof, see the sketch of the proof of Theorem 3.6 below, which includes this result as a special case.

Theorem 3.1. Let $\mu$ and $\nu$ be two compactly supported probability measures on $\mathbb{R}^{n}$. If $\mu$ is absolutely continuous with respect to the Lebesgue measure, then

(i) there exists a unique solution $T$ to the optimal transport problem with cost $c(x, y)=|x-y|^{2} / 2$

(ii) there exists a convex function $u: \mathbb{R}^{n} \rightarrow \mathbb{R}$ such that the optimal map $T$ is given by $T(x)=\nabla u(x)$ for $\mu$-a.e. $x$.

Furthermore, if $\mu(d x)=f(x) d x$ and $\nu(d y)=g(y) d y$, then $T$ is differentiable $\mu$-a.e. and

$$
|\operatorname{det}(\nabla T(x))|=\frac{f(x)}{g(T(x))} \quad \text { for } \mu \text {-a.e. } x \in \mathbb{R}^{n} \text {. }
$$


A remark which will be useful later is the following: the cost $|x-y|^{2} / 2$ is equivalent to the cost $-x \cdot y$. Indeed, for any transport map $S$ we have

$$
\int_{\mathbb{R}^{n}} \frac{|S(x)|^{2}}{2} d \mu(x)=\int_{\mathbb{R}^{n}} \frac{|y|^{2}}{2} d \nu(y)
$$

(this is a direct consequence of the condition $S_{\#} \mu=\nu$ ), hence

$$
\begin{aligned}
\int_{\mathbb{R}^{n}} & \frac{|x-S(x)|^{2}}{2} d \mu(x) \\
& =\int_{\mathbb{R}^{n}} \frac{|x|^{2}}{2} d \mu(x)+\int_{\mathbb{R}^{n}} \frac{|S(x)|^{2}}{2} d \mu(x)+\int_{\mathbb{R}^{n}}(-x \cdot S(x)) d \mu(x) \\
& =\int_{\mathbb{R}^{n}} \frac{|x|^{2}}{2} d \mu(x)+\int_{\mathbb{R}^{n}} \frac{|y|^{2}}{2} d \nu(y)+\int_{\mathbb{R}^{n}}(-x \cdot S(x)) d \mu(x),
\end{aligned}
$$

and since the first two integrals in the right-hand side are independent of $S$, we see that the two minimization problems

$$
\min _{S_{\#} \mu \nu} \int_{\mathbb{R}^{n}} \frac{|x-S(x)|^{2}}{2} d \mu(x) \quad \text { and } \quad \min _{S_{\#}=\nu} \int_{\mathbb{R}^{n}}(-x \cdot S(x)) d \mu(x)
$$

are equivalent.

3.2. Regularity theory for the quadratic cost: Brenier vs. Alexandrov solutions. Before starting the discussion of the regularity of optimal transport maps, let us recall some fact about second-order properties of convex functions (see for instance [110, Theorem 14.25]).

Theorem 3.2 (Alexandrov). Let $\Omega$ be a convex open set, and let $u: \Omega \rightarrow \mathbb{R}$ be $a$ convex function. Then, for a.e. $x$ in $\Omega, u$ is differentiable at $x$ and there exists a symmetric matrix $D^{2} u(x)$ such that

$$
u(y)=u(x)+\nabla u(x) \cdot(y-x)+\frac{1}{2} D^{2} u(x)(y-x) \cdot(y-x)+o\left(|y-x|^{2}\right) .
$$

In addition, at such points $\nabla u$ is differentiable with gradient given by $D^{2} u$, that is

$$
\nabla u(y)=\nabla u(x)+D^{2} u(x) \cdot(y-x)+o(|y-x|) \quad \forall y \in \operatorname{Dom}(\nabla u),
$$

where $\operatorname{Dom}(\nabla u)$ is the set of differentiability points of $u$.

Clearly, any convex function admits also a distributional Hessian $D_{\mathcal{D}}^{2} u$. Recalling that a positive distribution is a measure, it is simple to show that $D_{\mathcal{D}}^{2} u$ is a matrix-valued measure [44, Chapter 6]. Then one can show that the "pointwise" Hessian $D^{2} u$ defined in Theorem 3.2 coincides with the density of the absolutely continuous part of $D_{\mathcal{D}}^{2} u$ with respect to the Lebesgue measure; more precisely, the decomposition

$$
D_{\mathcal{D}}^{2} u=D^{2} u d x+\left(D_{\mathcal{D}}^{2} u\right)^{s}
$$

holds, where $\left(D_{\mathcal{D}}^{2} u\right)^{s}$ is singular with respect to the Lebesgue measure.

Let $X$ and $Y$ be two bounded smooth open sets in $\mathbb{R}^{n}$, and let $\mu(d x)=f(x) d x$ and $\nu(y)=g(y) d y$ be two probability measures with $f$ and $g$ such that $f=0$ in $\mathbb{R}^{2} \backslash X$ and $g=0$ in $\mathbb{R}^{2} \backslash Y$. We assume that $f$ and $g$ are bounded away from zero and infinity on $X$ and $Y$, respectively. By Brenier's Theorem, when the cost is given by $|x-y|^{2} / 2$, then the optimal transport map $T$ is the gradient of a convex function $u$. 
Hence, the Jacobian equation (3.3) combined with the fact that $\nabla u$ is differentiable a.e. (see Theorem 3.2) gives that $u$ solves the Monge-Ampère equation

$$
\operatorname{det}\left(D^{2} u(x)\right)=\frac{f(x)}{g(\nabla u(x))} \quad f d x \text {-a.e. }
$$

coupled with the "boundary condition"

$$
\nabla u(X)=Y
$$

(which corresponds to the fact that $T$ transports $f(x) d x$ onto $g(y) d y)$. We will say that the function $u$ is a Brenier solution of the Monge-Ampère equation.

In analogy to Definition 2.3. one can also define Alexandrov solutions in this context: we say that a convex function $v: X \rightarrow \mathbb{R}$ is an Alexandrov solution of (3.4) if

$$
|\partial v(E)|=\int_{E} \frac{f(x)}{g(\nabla v(x))} d x \quad \forall E \subset X,
$$

and to this class of solutions one may apply the regularity theory developed in Section 2.4. On the other hand, as observed by Caffarelli [19], even for smooth densities one cannot expect any general regularity result for Brenier solutions (equivalently, for optimal transport maps) without making some geometric assumptions on the support of the target measure. Indeed, let $n=2$ and suppose that $X=B_{1}$ is the unit ball centered at the origin and $Y=\left(B_{1}^{+}+e_{1}\right) \cup\left(B_{1}^{-}-e_{1}\right)$ is the union of two half-balls, where

$$
B_{1}^{+}:=\left(B_{1} \cap\left\{x_{1}>0\right\}\right), \quad B_{1}^{-}:=\left(B_{1} \cap\left\{x_{1}<0\right\}\right)
$$

and let $\left(e_{1}, e_{2}\right)$ denote the canonical basis of $\mathbb{R}^{2}$. Then, if $f=\frac{1}{|X|} \mathbf{1}_{X}$ and $g=\frac{1}{|Y|} \mathbf{1}_{Y}$, it is easily seen that the optimal map $T$ is given by

$$
T(x):= \begin{cases}x+e_{1} & \text { if } x_{1}>0 \\ x-e_{1} & \text { if } x_{1}<0\end{cases}
$$

which corresponds to the gradient of the convex function $u(x)=|x|^{2} / 2+\left|x_{1}\right|$.

Thus, as one could also show by an easy topological argument, in order to hope for a regularity result for $u$, we need at least to assume the connectedness of $Y$. But, starting from the above construction and considering a sequence of domains $X_{\varepsilon}^{\prime}$ where one adds a small strip of width $\varepsilon>0$ to glue together $\left(B_{1}^{+}+e_{2}\right) \cup$ $\left(B_{1}^{-}-e_{2}\right)$, one can also show that for $\varepsilon>0$ small enough the optimal map will still be discontinuous (see [19]).

The reason for this lack of regularity is the following. For Alexandrov solutions, whenever $f$ and $g$ are bounded away from zero and infinity on $X$ and $Y$, respectively, (3.6) implies that the multivalued map $x \mapsto \partial u(x)$ preserves the Lebesgue measure up to multiplicative constants, that is, the volumes of $E$ and $\partial u(E)$ are comparable for any Borel set $E \subset X$.

On the other hand, for Brenier solutions, (3.4) gives the same kind of information but only at points where $u$ is twice differentiable, so (3.4) may miss some singular part in the Monge-Ampère measure. This comes from the fact that the optimal map can only see the regions where $f$ and $g$ live, and for Brenier solutions one can show that

$$
\int_{E} f(x) d x=\int_{\partial u(E)} g(y) d y \quad \forall E \subset X .
$$


Thus, if $f$ and $g$ are bounded away from zero and infinity on $X$ and $Y$, respectively, we only have

$$
|E| \simeq|\partial u(E) \cap Y| \quad \forall E \subset X
$$

(and not $|E| \simeq|\partial u(E)|$ as in the Alexandrov case), which means that we do not have a full control on the Monge-Ampère measure of $u$.

In the counterexample above what happens is the following: when $x=\left(0, x_{2}\right)$, then $\partial u(x)=[-1,1] \times\left\{x_{2}\right\}$, hence $\partial u$ maps the segment $\{0\} \times[-1,1]$ onto the square $[-1,1]^{2}$, but in the latter square $g$ has no mass. Notice that in this case the determinant of $D^{2} u$ is equal to 1 a.e. inside $B_{1}$ (so $u$ is a Brenier solution with right-hand side 1 ) but the Monge-Ampère measure of $u$ is equal to $d x\left\llcorner B_{1}+\right.$ $\mathcal{H}^{1}\left\llcorner\left(\left\{x_{1}=0\right\} \cap B_{1}\right)\right.$ (compare with Example 2.2).

Hence, in order to avoid this kind of counterexample, one should make sure that the target mass always cover the image of $\partial u(X)$, and a way to ensure this is that $Y$ is convex. Indeed, as shown by Caffarelli [19], if $Y$ is convex, then $\partial u(X) \subset \bar{Y}$ (see the proof of Theorem 3.3 below) and any Brenier solution is an Alexandrov solution. In particular the regularity results from Section 2.4 apply whenever $f$ and $g$ are strictly positive on their respective support (since, in that case, the right-hand side in (3.4) is bounded away from zero and infinity) and we have the following (see also [19,22]):

Theorem 3.3. Let $X, Y \subset \mathbb{R}^{n}$ be two bounded open sets, let $f: X \rightarrow \mathbb{R}^{+}$and $g: Y \rightarrow \mathbb{R}^{+}$be two probability densities, respectively, bounded away from zero and infinity on $X$ and $Y$, and denote by $T=\nabla u: X \rightarrow Y$ the unique optimal transport map sending $f$ onto $g$ for the cost $|x-y|^{2} / 2$. Assume that $Y$ is convex. Then

- $T \in C_{\mathrm{loc}}^{0, \alpha}(X) \cap W_{\mathrm{loc}}^{1,1+\varepsilon}(X)$;

- if in addition $f \in C_{\mathrm{loc}}^{k, \beta}(X)$ and $g \in C_{\mathrm{loc}}^{k, \beta}(Y)$ for some $\beta \in(0,1)$, then $T \in C_{\mathrm{loc}}^{k+1, \beta}(X)$;

- furthermore, if $f \in C^{k, \beta}(\bar{X}), g \in C^{k, \beta}(\bar{Y})$ and both $X$ and $Y$ are smooth and uniformly convex, then $T: \bar{X} \rightarrow \bar{Y}$ is a global diffeomorphism of class $C^{k+1, \beta}$.

Sketch of the proof. As we explained, at least for the interior regularity, the key step in the proof is to show that, when $Y$ is convex, Brenier solutions are Alexandrov solutions. Let us briefly sketch the proof of this fact.

Step 1. For any set $A \subset X$, it holds that

$$
\mu_{u}(A) \geq \int_{A} \frac{f(x)}{g(\nabla u(x))} d x .
$$

This is a general fact that does not need the convexity of $Y$. Indeed, for any set $A \subset X$,

$$
\partial u(A) \supset \nabla u(A \cap \operatorname{Dom}(\nabla u)) .
$$

Then, by the Alexandrov theorem, since $\nabla u$ is differentiable almost everywhere, by the Area Formula [46, Corollary 3.2.20] and (3.3) we get

$$
\mu_{u}(A)=|\partial u(A)| \geq|\nabla u(A \cap \operatorname{Dom}(\nabla u))|=\int_{A} \operatorname{det} D^{2} u d x=\int_{A} \frac{f(x)}{g(\nabla u(x))} d x .
$$

Step 2. If $\partial u(A) \subset Y$ up to a set of measure zero, then

$$
\mu_{u}(A)=\int_{A} \frac{f(x)}{g(\nabla u(x))} d x .
$$


To see this, notice that, for all $A \subset X$,

$$
A \cap \operatorname{Dom}(\nabla u) \subset(\nabla u)^{-1}(\partial u(A))
$$

and

$$
\begin{aligned}
& (\nabla u)^{-1}(\partial u(A) \cap Y) \backslash A \\
& \subset(\nabla u)^{-1}\left(\left\{y \in Y: \text { there exist } x_{1}, x_{2}, x_{1} \neq x_{2}\right.\right. \\
& \text { such that } \left.\left.y \in \partial u\left(x_{1}\right) \cap \partial u\left(x_{2}\right)\right\}\right) \\
& \subset(\nabla u)^{-1}\left(\left\{\text { points of nondifferentiability of } u^{*}\right\} \cap Y\right),
\end{aligned}
$$

where $u^{*}$ is the convex conjugate of $u$; see for instance [64, Lemma 1.1.12]. Since any convex function is differentiable almost everywhere, by our assumptions on the densities and the transport relation $(\nabla u)_{\sharp}(f d x)=g d y$, we infer that

$$
\left|(\nabla u)^{-1}(\partial u(A) \cap Y) \cap(X \backslash A)\right|=0 .
$$

Since $\partial u(A) \subset Y$ a.e. and $f$ vanishes outside $X$, using again that $(\nabla u)_{\sharp}(f d x)=$ $g d y$, we get

$$
\begin{aligned}
|\partial u(A)| & =\int_{\partial u(A) \cap Y} \frac{g(y)}{g(y)} d y=\int_{(\nabla u)^{-1}(\partial u(A))} \frac{f(x)}{g(\nabla u(x))} d x \\
& =\int_{A} \frac{f(x)}{g(\nabla u(x))} d x+\int_{(\nabla u)^{-1}(\partial u(A)) \backslash A} \frac{f(x)}{g(\nabla u(x))} d x=\int_{A} \frac{f(x)}{g(\nabla u(x))} d x .
\end{aligned}
$$

Step $3(\partial u(X) \subset Y)$. Recall that, as a general fact for convex functions,

$\partial u(x)=\operatorname{Conv}\left(\left\{p:\right.\right.$ there exist $x_{k} \in \operatorname{Dom}(\nabla u)$ with $x_{k} \rightarrow x$ and $\left.\left.\nabla u\left(x_{k}\right) \rightarrow p\right\}\right) ;$

see [28, Theorem 3.3.6]. Since $(\nabla u)_{\sharp}(f d x)=g d y$, the set of $x \in X \cap \operatorname{Dom}(\nabla u)$ such that $\nabla u(x) \in Y$ is of full measure in $X$, in particular it is dense. From this, (3.7), and the convexity of $Y$, one immediately deduces that

$$
\partial u(X) \subset \overline{\operatorname{Conv}(Y)}=\bar{Y} .
$$

Since the measure of $\partial Y$ is zero, using the previous step, we conclude that $u$ is an Alexandrov solution of

$$
\operatorname{det} D^{2} u=\frac{f}{g \circ \nabla u} .
$$

Finally, to apply the results from Section 2.4 one then has to show that $u$ is strictly convex inside $X$; see [19] for more details.

A natural question is what happens when one removes the convexity assumption on the target. As shown in 51] (see also [49] for a more precise description of the singular set in two dimensions), in this case one can prove that the optimal transport map is smooth outside a closed set of measure zero. More precisely, the following holds.

Theorem 3.4. Let $X, Y \subset \mathbb{R}^{n}$ be two bounded open sets, let $f: X \rightarrow \mathbb{R}^{+}$and $g: Y \rightarrow \mathbb{R}^{+}$be two probability densities, respectively, bounded away from zero and infinity on $X$ and $Y$, and denote by $T=\nabla u: X \rightarrow Y$ the unique optimal transport map sending $f$ onto $g$ for the cost $|x-y|^{2} / 2$. Then there exist two relatively closed sets $\Sigma_{X} \subset X, \Sigma_{Y} \subset Y$ of measure zero such that $T: X \backslash \Sigma_{X} \rightarrow Y \backslash \Sigma_{Y}$ is a 
homeomorphism of class $C_{\mathrm{loc}}^{0, \alpha}$ for some $\alpha>0$. In addition, if $c \in C_{\mathrm{loc}}^{k+2, \alpha}(X \times Y)$, $f \in C_{\mathrm{loc}}^{k, \alpha}(X)$, and $g \in C_{\mathrm{loc}}^{k, \alpha}(Y)$ for some $k \geq 0$ and $\alpha \in(0,1)$, then $T: X \backslash \Sigma_{X} \rightarrow$ $Y \backslash \Sigma_{Y}$ is a diffeomorphism of class $C_{\mathrm{loc}}^{k+1, \alpha}$.

Sketch of the proof. As explained above, when $Y$ is not convex there could be points $x \in X$ such that $\partial u(x) \nsubseteq Y$, and on these points we have no control on the MongeAmpère measure of $u$. Let us defin 10

$$
\operatorname{Reg}_{X}:=\{x \in X: \partial u(x) \subset Y\}, \quad \Sigma_{X}:=X \backslash \operatorname{Reg}_{X} .
$$

By the continuity property of the subdifferential [28, Proposition 3.3.4], it is immediate to see that $\operatorname{Reg}_{X}$ is open. Moreover it follows from the condition $(\nabla u)_{\sharp}(f d x)=$ $g d y$ that $\nabla u(x) \in Y$ for a.e. $x \in X$, hence $\left|\Sigma_{X}\right|=0$. Thus, arguing as in Step 2 in the proof of Theorem 3.3 one can show that $u$ is a strictly convex Alexandrov solution on $\operatorname{Reg}_{X}$, so one can apply the regularity results of Section 2

3.3. The partial transport problem. Let us remark that, in addition to the situation described in Section 2.5, the regularity theory for optimal transport maps with right-hand side only bounded away from zero and infinity appears in other situations. An important example is provided by the "partial transport problem" described here.

Given two densities $f$ and $g$, one wants to transport a fraction

$$
m \in\left[0, \min \left\{\|f\|_{L^{1}},\|g\|_{L^{1}}\right\}\right]
$$

of the mass of $f$ onto $g$ minimizing the transportation cost $c(x, y)=|x-y|^{2} / 2$. The existence and uniqueness of minimizers it is a very nontrivial problem.

In particular, one can notice that in general uniqueness of minimizers does not hold: if $m \leq \int_{\mathbb{R}^{n}} \min \{f, g\}$, the family of minimizers is simply given by choosing any fraction of mass of $\min \{f, g\}$ with total volume $m$ and just send this mass to itself with zero cost.

To ensure uniqueness, in 25] the authors assumed $f$ and $g$ to have disjoint supports. Under this assumption they were able to prove (as in the classical optimal transport problem) that there exists a convex function $u$ such that the unique minimizer is given by $\nabla u$. This function $u$ is also shown to solve in a weak sense a "Monge-Ampère double obstacle problem". Then, strengthening the disjointness assumption into the hypothesis on the existence of a hyperplane separating the supports of the two measures, the authors prove a semiconvexity result on the free boundaries. Furthermore, under some classical regularity assumptions on the measures and on their supports, local $C^{1, \alpha}$-regularity of $u$, and on the free boundaries of the active regions, is shown.

In 48 the second author studied what happens if one removes the disjointness assumption. Although minimizers are nonunique for $m<\int_{\mathbb{R}^{n}} \min \{f, g\}$ (but in this case the set of minimizers can be trivially described, as mentioned above), uniqueness holds for any $m \geq \int_{\mathbb{R}^{n}} \min \{f, g\}$. Moreover, exactly as in [25, the unique minimizer is given by the gradient of a convex function.

An important fact proved in [48] is that both the source and the target mass dominate the common mass $\min \{f, g\}$ (that is, the common mass is both source and target). This property, which has an interest on its own, implies that the

\footnotetext{
${ }^{10}$ Actually, in 49, 51] the regular set is defined in a slightly different way, and it is in principle smaller. However, the advantage of that definition is that it allows for a more refined analysis of the singular set (see 49]).
} 
free boundaries never enter inside the intersection of the supports of $f$ and $g$, a fact which plays a crucial role in the regularity of the free boundaries. Indeed, it is shown that the free boundaries have zero Lebesgue measure (under some mild assumptions on the supports of the two densities). As a consequence of this fact, whenever the support of $g$ is assumed to be convex and $f$ and $g$ are bounded away from zero and infinity on their respective support, Theorem 3.3 allows one to deduce local $C^{0, \alpha}$-regularity of the transport map and to show that it extends to an homeomorphism up to the boundary if both supports are assumed to be strictly convex. Further regularity results on the free boundaries were obtained later in [47] and 66 .

On the other hand, in this situation where the supports of $f$ and $g$ can intersect, something new happens: in the classical optimal transport problem, by assuming $C^{\infty}$-regularity on the density of $f$ and $g$ (together with some convexity assumption on their supports), Theorem 3.3 ensures that the transport map is $C^{\infty}$ also. In contrast with this, in [48] the author showed that $C_{\text {loc }}^{0, \alpha}$ regularity is optimal: one can find two $C^{\infty}$-densities on $\mathbb{R}$, supported on two bounded intervals and bounded away from zero on their supports, such that the transport map is not $C^{1}$.

3.4. The case of a general cost. After Theorem 3.1 many researchers started to work on the problem of showing existence of optimal maps in the case of more general costs, both in the Euclidean setting and in the case of Riemannian manifolds. Since at least locally any Riemannian manifold looks like $\mathbb{R}^{n}$, here we shall focus on the case of $\mathbb{R}^{n}$, and then in Section 4.3 we will discuss some of the nontrivial issues that may arise on manifolds (for instance, due to the presence of the cut-locus).

We introduce first some conditions on the cost. Here and in the sequel, $X$ and $Y$ denote two open subsets of $\mathbb{R}^{n}$.

(C0) The cost function $c: X \times Y \rightarrow \mathbb{R}$ is of class $C^{4}$ with $\|c\|_{C^{4}(X \times Y)}<\infty$.

(C1) For any $x \in X$, the map $Y \ni y \mapsto D_{x} c(x, y) \in \mathbb{R}^{n}$ is injective.

(C2) For any $y \in Y$, the map $X \ni x \mapsto D_{y} c(x, y) \in \mathbb{R}^{n}$ is injective.

(C3) $\operatorname{det}\left(D_{x y} c\right)(x, y) \neq 0$ for all $(x, y) \in X \times Y$.

Note that these conditions are satisfied by the cost $-x \cdot y$ when $X$ and $Y$ are bounded.

We now introduce the notion of $c$-convexity, which will play an important role in all of our discussion. A function $\psi: X \rightarrow \mathbb{R} \cup\{+\infty\}$ is c-convex if

$$
\psi(x)=\sup _{y \in Y}\left[\psi^{c}(y)-c(x, y)\right] \quad \forall x \in X,
$$

where $\psi^{c}: Y \rightarrow \mathbb{R} \cup\{-\infty\}$ is given by

$$
\psi^{c}(y):=\inf _{x \in X}[\psi(x)+c(x, y)] \quad \forall y \in Y .
$$

For a $c$-convex function $\psi$, we define its $c$-subdifferential at $x$ as

$$
\partial_{c} \psi(x):=\left\{y \in Y: \psi(x)=\psi^{c}(y)-c(x, y)\right\}
$$

or, equivalently,

$$
\partial_{c} \psi(x):=\{y \in \bar{Y}: \psi(z) \geq-c(z, y)+c(x, y)+\psi(x) \quad \forall z \in X\} .
$$

${ }^{11}$ The terminology comes from the fact that, in the case $c(x, y)=-x \cdot y$ (which is equivalent to $|x-y|^{2} / 2$, see Section 3.1), $c$-convexity corresponds to the classical notion of convexity (since in that case $\psi$ is a supremum of linear functions). Analogously, the $c$-subdifferential defined in (3.9) corresponds to the classical subdifferential for convex functions. 
We also define the Fréchet subdifferential of $\psi$ at $x$ as

$$
\partial^{-} \psi(x):=\left\{p \in \mathbb{R}^{n}: \psi(z) \geq u(x)+p \cdot(z-x)+o(|z-x|)\right\} .
$$

It is easy to check that, if $c$ is of class $C^{1}$, then the following inclusion holds:

$$
y \in \partial_{c} \psi(x) \quad \Longrightarrow \quad-D_{x} c(x, y) \in \partial^{-} \psi(x) .
$$

In addition, if $c$ satisfies (C0)-(C2), then we can define the $c$-exponential map 12

Using (3.12), we can rewrite (3.11) as

$$
\partial_{c} \psi(x) \subset c-\exp _{x}\left(\partial^{-} \psi(x)\right) .
$$

Let us point out that, when $c(x, y)=-x \cdot y, \psi$ is convex and $\partial_{c} \psi=\partial \psi$, the above inclusion is an equality (since any local supporting hyperplane is a global support). However, for a general cost function $c$, the above inclusion may be strict when $\psi$ is nondifferentiable. As we will discuss in Section 4, equality in (3.13) for every $c$-convex function is necessary for regularity of optimal maps between smooth densities. Notice that, if $c \in C^{1}$ and $Y$ is bounded, it follows immediately from (3.8) that $c$-convex functions are Lipschitz, so in particular they are differentiable almost everywhere.

Remark 3.5. If $c$ satisfies (C0) and $Y$ is bounded, then it follows from (3.8) that $\psi$ is semiconvex (i.e., there exists a constant $C>0$ such that $\psi+C|x|^{2} / 2$ is convex, see for instance [45]). In particular, by Theorem [3.2, $c$-convex functions are twice differentiable almost everywhere.

The following is a basic result in optimal transport theory.

Theorem 3.6. Let $c: X \times Y \rightarrow \mathbb{R}$ satisfy (C0)-(C1). Given two probability densities $f$ and $g$ supported on $X$ and $Y$, respectively, there exists a c-convex function $u: X \rightarrow \mathbb{R}$ such that the map $T_{u}: X \rightarrow Y$ defined by $T_{u}(x):=c-\exp _{x}(\nabla u(x))$ is the unique optimal transport map sending $f$ onto $g$. If in addition (C2) holds, then $T_{u}$ is injective $f d x$-a.e.,

$$
\left|\operatorname{det}\left(\nabla T_{u}(x)\right)\right|=\frac{f(x)}{g\left(T_{u}(x)\right)} \quad f d x \text {-a.e. },
$$

and its inverse is given by the optimal transport map sending $g$ onto $f$.

Notice that, in the particular case $c(x, y)=-x \cdot y, c$-convex functions are convex and the above result corresponds to Theorem 3.1. We give here a sketch of the proof of Theorem 3.6, referring to [110, Chapter 10] for more details.

Sketch of the proof. There are several ways to establish this result. One possibility is to go through the following steps:

Step 1 (Solve the Kantorovich problem). Following Kantorovich [70,71, we consider the following relaxed problem. Instead of minimizing the transportation cost among all transport maps (see (3.2)), we consider instead the problem

$$
\inf _{\pi \in \Pi(f, g)}\left\{\int_{X \times Y} c(x, y) d \pi(x, y)\right\}
$$

\footnotetext{
${ }^{12}$ The name "c-exponential" is motivated by the fact that, when $c$ is given by the squared Riemannian distance on a manifold, the $c$-exponential map coincides with the classical exponential map in Riemannian geometry.
} 
where $\Pi(f, g)$ denotes the set of all probability measures $\pi$ on $X \times Y$ whose marginals are $f d x$ and $g d y$, i.e.,

$$
\int_{X \times Y} h(x) d \pi(x, y)=\int_{X} h(x) f(x) d x, \quad \int_{X \times Y} h(y) d \pi(x, y)=\int_{Y} h(y) g(y) d y,
$$

for all $h: M \rightarrow \mathbb{R}$ bounded continuous.

The connection between the formulation of Kantorovich and that of Monge is the following: any transport map $T$ induces the plan defined by $(\operatorname{Id} \times T)_{\#} \mu$ which is concentrated on the graph of $T$. Conversely, if a transport plan is concentrated on the graph of a measurable function $T$, then it is induced by this map.

By weak compactness of the set $\Pi(f, g)$ and continuity of the function $\pi \mapsto$ $\int c(x, y) d \pi$, it is simple to show the existence of a minimizer $\bar{\pi}$ for (3.14); so to prove the existence of a solution to the Monge problem it suffices to show that $\bar{\pi}$ is concentrated on the graph of a measurable map $T$, i.e.,

$$
y=T(x) \quad \text { for } \bar{\pi} \text {-almost every }(x, y) \text {. }
$$

Once this fact is proved, the uniqueness of optimal maps will follow from the observation that, if $T_{1}$ and $T_{2}$ are optimal, then $\pi_{1}:=\left(\operatorname{Id} \times T_{1}\right)_{\#} \mu$ and $\pi_{2}:=\left(\operatorname{Id} \times T_{2}\right)_{\#} \mu$ are both optimal plans, so by linearity $\bar{\pi}=\frac{1}{2}\left(\pi_{1}+\pi_{2}\right)$ is optimal. If it is concentrated on a graph, this implies that $T_{1}$ and $T_{2}$ must coincide $f d x$-a.e., proving the desired uniqueness.

Step 2 (The support of $\bar{\pi}$ is c-cyclically monotone). A set $S \subset X \times Y$ is called c-cyclically monotone if, for all $N \in \mathbb{N}$, for all $\left\{\left(x_{i}, y_{i}\right)\right\}_{0 \leq i \leq N} \subset S$, one has

$$
\sum_{i=0}^{N} c\left(x_{i}, y_{i}\right) \leq \sum_{i=0}^{N} c\left(x_{i}, y_{i+1}\right),
$$

where by convention $y_{N+1}=y_{0}$. The above definition means heuristically that sending the point $x_{i}$ to the point $y_{i}$ for $i=0, \ldots, N$ is globally less expensive than sending the point $x_{i}$ to the point $y_{i+1}$. It is therefore intuitive that, since $\bar{\pi}$ is optimal, its support is $c$-cyclically monotone (see [61] or [110, Chapter 5] for a proof).

Step 3 (Any $c$-cyclically monotone set is contained in the $c$-subdifferential of a $c$-convex function). A proof of this fact (which is due to Rockafellar for $c(x, y)=$ $-x \cdot y$, and Rüschendorf for the general case) consists in constructing explicitly a $c$-convex function which does the work: given $S$ c-cyclically monotone, we define

$$
\begin{array}{r}
u(x):=\sup _{N \in \mathbb{N}} \sup _{\left\{\left(x_{i}, y_{i}\right)\right\}_{1 \leq i \leq N} \subset S}\left\{\left[c\left(x_{0}, y_{0}\right)-c\left(x_{1}, y_{0}\right)\right]+\left[c\left(x_{1}, y_{1}\right)-c\left(x_{2}, y_{1}\right)\right]\right. \\
\left.+\ldots+\left[c\left(x_{N}, y_{N}\right)-c\left(x, y_{N}\right)\right]\right\},
\end{array}
$$

where $\left(x_{0}, y_{0}\right)$ is arbitrarily chosen in $S$. It can be checked easily that, with this definition, $u$ is $c$-convex and $S \subset \bigcup_{x \in X}\left(\{x\} \times \partial_{c} u(x)\right)$ (see for instance [110, Proof of Theorem 5.10]).

Step 4 ( $\bar{\pi}$ is concentrated on a graph). Applying Steps 2 and 3, we know that the support of $\bar{\pi}$ is contained in the $c$-subdifferential of a $c$-convex function $u$. Moreover, since $u$ is the supremum of the family of uniformly Lipschitz functions $c(\cdot, y)+\lambda_{y}$ (see (3.8) ), it is Lipschitz, hence differentiable almost everywhere. Being the first 
marginal of $\bar{\pi}$ absolutely continuous with respect to the Lebesgue measure, we deduce that, for $\bar{\pi}$-almost every $(x, y), u$ is differentiable at $x$.

Now, let us fix a point $(\bar{x}, \bar{y}) \in \operatorname{supp}(\pi)$ such that $u$ is differentiable at $\bar{x}$. To prove that $\bar{\pi}$ is concentrated on a graph, it suffices to prove that $\bar{y}$ is uniquely determined as a function of $\bar{x}$. To this aim, we observe that, since the support of $\bar{\pi}$ is contained in the $c$-subdifferential of $u$, we have $\bar{y} \in \partial_{c} u(\bar{x})$, and this implies that the function $x \mapsto u(x)+c(x, \bar{y})$ attains a minimum at $\bar{x}$. Hence, being $u$ is differentiable at $\bar{x}$ we obtain

$$
\nabla u(\bar{x})+\nabla_{x} c(\bar{x}, \bar{y})=0 .
$$

Recalling (3.12) this implies that

$$
\bar{y}=c-\exp _{\bar{x}}(\nabla u(\bar{x}))=: T_{u}(\bar{x}) .
$$

This proves that $\bar{\pi}$ is concentrated on the graph of the map $T_{u}$, as desired.

This concludes the first part of the theorem. The invertibility of $T_{u}$ under (C2) follows by a simple argument based on the uniqueness of the minimizer of (3.14); see [9, Remark 6.2.11] for more details.

In the case when $X=Y=(M, g)$ is a compact Riemannian manifold, one has to face the additional difficulty that the cost function $c=d^{2} / 2$ is not smooth everywhere. However, by exploring the superdifferentiability properties of the squared Riemannian distance function (i.e., the fact that, for any $y \in M$, the Fréchet subdifferential of $-d^{2}(\cdot, y)$ is nonempty at every point), McCann was able to extend the theorem above the the case of compact Riemannian manifolds 88] (see also [45] for a more general result):

Theorem 3.7. Let $M$ be a smooth compact Riemannian manifold, and consider the cost $c=d^{2} / 2$, with $d$ being the Riemannian distance. Given two probability densities $f$ and $g$ supported on $M$, there exists a c-convex function $u: M \rightarrow \mathbb{R} \cup\{+\infty\}$ such that $T_{u}(x)=\exp _{x}(\nabla u(x))$ is the unique optimal transport map sending $f$ onto $g$.

\section{A Class of Monge-Ampère type EQuations}

As shown in Theorem 3.6, whenever the cost function satisfies (C0)-(C1) then the unique optimal transport map sending $f$ onto $g$ is given by $T_{u}(x)=$ $c$ - $\exp _{x}(\nabla u(x))$. Furthermore, if $c$ satisfies (C2), then

$$
\left|\operatorname{det}\left(\nabla T_{u}(x)\right)\right|=\frac{f(x)}{g\left(T_{u}(x)\right)} \quad \text { a.e. }
$$

Now, since $\left\{c-\exp _{x}(\nabla u(x))\right\}=\partial_{c} u(x)$ at every differentiability point (see (3.13)), it follows that $z \mapsto u(z)+c\left(z, c-\exp _{x}(\nabla u(x))\right)$ attains a minimum at $z=x$. Hence, whenever $u$ is twice differentiable at $x$ (that is at almost every point, see Remark 3.5), we get

$$
D^{2} u(x)+D_{x x} c\left(x, c-\exp _{x}(\nabla u(x))\right) \geq 0 .
$$

Hence, rewriting the relation $T_{u}(x)=c-\exp _{x}(\nabla u(x))$ as

$$
-D_{x} c\left(x, T_{u}(x)\right)=\nabla u(x)
$$


and differentiating the above equation with respect to $x$, using (4.1) and (4.2) we obtain

$$
\begin{aligned}
\operatorname{det}( & \left.D^{2} u(x)+D_{x x} c\left(x, c-\exp _{x}(\nabla u(x))\right)\right) \\
& =\left|\operatorname{det}\left(D_{x y} c\left(x, c-\exp _{x}(\nabla u(x))\right)\right)\right| \frac{f(x)}{g\left(c-\exp _{x}(\nabla u(x))\right)}
\end{aligned}
$$

at every point $x$ where $u$ it is twice differentiable.

Hence $u$ solves a Monge-Ampère type equation of the form (1.2) with

$$
\mathcal{A}(x, \nabla u(x)):=-D_{x x} c\left(x, c-\exp _{x}(\nabla u(x))\right) .
$$

Notice that if (C3) holds and $f$ and $g$ are bounded away from zero and infinity on their respective supports, then the right-hand side in 4.3 is bounded away from zero and infinity on the support of $f$. As we will see below, some structural conditions on $\mathcal{A}$ (or equivalently on the $\operatorname{cost} c$ ) must be imposed to obtain regularity results.

The breakthrough in the study of regularity of optimal transport maps came with the paper of Ma, Trudinger, and Wang [87. (whose roots lie in an earlier work of Wang on the reflector antenna problem [112), where the authors found a mysterious fourth-order condition on the cost functions, which turned out to be sufficient to prove the regularity of $u$. The idea was to differentiate twice equation (4.3) in order to get a linear PDE for the second derivatives of $u$, and then to try to show an a priori estimate on the second derivatives of $u$; compare with Theorem 2.12. In this computation, one obtains a certain moment with a term which needs to have a sign in order to conclude the desired a priori estimate. This term is what is now called the "Ma-Trudinger-Wang tensor" (in short MTW tensor):

$$
\begin{aligned}
\mathfrak{S}_{(x, y)}(\xi, \eta) & :=D_{p_{\eta} p_{\eta}}^{2} \mathcal{A}(x, p)[\xi, \xi] \\
& =\sum_{i, j, k, l, p, q, r, s}\left(c_{i j, p} c^{p, q} c_{q, r s}-c_{i j, r s}\right) c^{r, k} c^{s, l} \xi^{i} \xi^{j} \eta^{k} \eta^{l}, \quad \xi, \eta \in \mathbb{R}^{n} .
\end{aligned}
$$

In the above formula the cost function is evaluated at $(x, y)=\left(x, c-\exp _{x}(p)\right)$, and we used the notation $c_{j}=\frac{\partial c}{\partial x^{j}}, c_{j k}=\frac{\partial^{2} c}{\partial x^{j} \partial x^{k}}, c_{i, j}=\frac{\partial^{2} c}{\partial x^{i} \partial y^{j}}, c^{i, j}=\left(c_{i, j}\right)^{-1}$. The condition to impose on $\mathfrak{S}_{(x, y)}(\xi, \eta)$ is

$$
\mathfrak{S}_{(x, y)}(\xi, \eta) \geq 0 \quad \text { whenever } \xi \perp \eta
$$

(this is called the MTW condition). Actually, it is convenient to introduce a more general definition:

Definition 4.1. Given $K \geq 0$, we say that $c$ satisfies the $\operatorname{MTW}(K)$ condition if, for all $(x, y) \in(X \times Y)$ and for all $\xi, \eta \in \mathbb{R}^{n}$,

$$
\mathfrak{S}_{(x, y)}(\xi, \eta) \geq K|\xi|^{2}|\eta|^{2} \quad \text { whenever } \xi \perp \eta \text {. }
$$

Under this hypothesis and a geometric condition on the supports of the measures (which is the analogue of the convexity assumption of Caffarelli), Ma, Trudinger, and Wang could prove the following result [87, 108, 109] (see also [103]):

Theorem 4.2. Let $c: X \times Y \rightarrow \mathbb{R}$ satisfy (C0)-(C3). Assume that $\mathrm{MTW}(0)$ holds and that $f$ and $g$ are smooth and bounded away from zero and infinity on 
their respective supports $X$ and $Y$. Also, suppose that:

(a) $X$ and $Y$ are smooth;

(b) $D_{x} c(x, Y)$ is uniformly convex for all $x \in X$;

(c) $D_{y} c(X, y)$ is uniformly convex for all $y \in Y$.

Then $u \in C^{\infty}(\bar{X})$ and $T: \bar{X} \rightarrow \bar{Y}$ is a smooth diffeomorphism, where $T(x)=$ $c-\exp _{x}(\nabla u(x))$.

Sketch of the proof. For simplicity here we treat the simpler case when $\operatorname{MTW}(K)$ holds for some $K>0$. This stronger MTW condition is actually the one originally used in [87, 109. The general case $K=0$ is treated in 108, where the authors relax the stronger assumption by applying a barrier argument.

As we pointed out in Remark 2.1 for the classical Monge-Ampère equation, the key point to obtain existence of smooth solutions was to show an a priori estimate on second derivatives of solutions. The same is true in the case of (4.3). Indeed, once we know an a priori $C^{2}$-bound on smooth solutions of 4.3), this equation becomes uniformly elliptic (in the sense that the linearized operator is uniformly elliptic, see Remark 2.1) and one can run a parallel strategy to the one described in Section 2.2 to get the existence of smooth solutions (see 108 for more details).

Let us start from a smooth (say $C^{4}$ ) solution of (4.3), coupled with the boundary condition $T(X)=Y$, where $T(x)=c$ - $\exp _{x}(\nabla u(x))$. Our goal here is to show an interior universal bound for the second derivatives of $u$.

We observe that, since $T(x)=c-\exp _{x}(\nabla u(x))$, we have

$$
|\nabla u(x)|=\left|\nabla_{x} c(x, T(x))\right| \leq C,
$$

hence $u$ is globally Lipschitz, with a uniform Lipschitz bound. Define

$$
w_{i j}:=D_{x^{i} x^{j}} u+D_{x^{i} x^{j}} c\left(x, c-\exp _{x}(\nabla u(x))\right) .
$$

(Recall that, by the $c$-convexity of $u$ and (4.3), $\left(w_{i j}\right)$ is positive definite.) Then (4.3) can be written as

$$
\operatorname{det}\left(w_{i j}\right)=h(x, \nabla u(x))
$$

or equivalently

$$
\log \left(\operatorname{det}\left(w_{i j}\right)\right)=\varphi,
$$

with $\varphi(x):=\log (h(x, \nabla u(x)))$. By differentiating the above equation and using the convention of summation over repeated indices, we get

$$
\begin{gathered}
w^{i j} w_{i j, k}=\varphi_{k}, \\
w^{i j} w_{i j, k k}=\varphi_{k k}+w^{i s} w^{j t} w_{i j, k} w_{s t, k} \geq \varphi_{k k},
\end{gathered}
$$

where $\left(w^{i j}\right)$ denotes the inverse of $\left(w_{i j}\right)$ and we use the notation $\psi_{k}=\frac{\partial}{\partial x^{k}} \psi$, $w_{i j, k}=\frac{\partial}{\partial x^{k}} w_{i j}, T_{s, k}=\frac{\partial}{\partial x^{k}} T_{s}$, and so on. Then the above equations become

$$
\begin{gathered}
w^{i j}\left[u_{i j k}+c_{i j k}+c_{i j, s} T_{s, k}\right]=\varphi_{k}, \\
w^{i j}\left[u_{i j k k}+c_{i j k k}+2 c_{i j k, s} T_{s, k}+c_{i j, s} T_{s, k k}+c_{i j, s t} T_{s, k} T_{t, k}\right] \geq \varphi_{k k} .
\end{gathered}
$$

We fix now $\bar{x} \in X$, we take $\eta$ a cut-off function around $\bar{x}$, and define the function $G: X \times \mathbb{S}^{n-1} \rightarrow \mathbb{R}$

$$
G(x, \xi):=\eta(x)^{2} w_{\xi \xi}, \quad w_{\xi \xi}:=\sum_{i j} w_{i j} \xi^{i} \xi^{j} .
$$


We want to show that $G$ is uniformly bounded by a universal constant $C$, depending only on $\operatorname{dist}(\bar{x}, \partial X), n$, the cost function, and the function $h(x, p)$. (Observe that $G \geq 0$, since $\left(w_{i j}\right)$ is positive definite.) In fact, this will imply that

$$
\eta(x)^{2}\left|D^{2} u(x)+D_{x x} c\left(x, c-\exp _{x}(\nabla u(x))\right)\right| \leq C,
$$

and since $\nabla u(x)$ is bounded and $c$ is smooth, the above equation gives that $\left|D^{2} u\right|$ is locally uniformly bounded by a universal constant, which is the desired a priori estimate.

To prove the bound on $G$, the strategy is the same as Theorem 2.12, let $x_{0} \in X$, and let $\xi_{0} \in \mathbb{S}^{n-1}$ be a point where $G$ attains its maximum. By a rotation of coordinates, one can assume $\xi_{0}=e_{1}$. Then at $x_{0}$ we have

$$
\begin{gathered}
0=(\log G)_{i}=\frac{w_{11, i}}{w_{11}}+2 \frac{\eta_{i}}{\eta}, \\
(\log G)_{i j}=\frac{w_{11, i j}}{w_{11}}+2 \frac{\eta_{i j}}{\eta}-6 \frac{\eta_{i} \eta_{j}}{\eta^{2}} .
\end{gathered}
$$

Since the above matrix is nonpositive, we get

$$
0 \geq w_{11} w^{i j}(\log G)_{i j}=w^{i j} w_{11, i j}+2 \frac{w_{11}}{\eta} w^{i j} \eta_{i j}-6 w_{11} w^{i j} \frac{\eta_{i} \eta_{j}}{\eta^{2}} .
$$

We further observe that, differentiating the relation $\nabla u=-D_{x} c(x, T(x))$, we obtain the relation

$$
w_{i j}=c_{i, k} T_{k, j} .
$$

This gives in particular $T_{k, j}=c^{k, i} w_{i j}$ (which implies $|\nabla T| \leq C w_{11}$ ), and allows us to write derivatives of $T$ in terms of that of $w$ and $c$.

The idea is now to start from (4.9), and to combine the information coming from (4.6), (4.7), (4.8), (4.10), to end up with a inequality of the form

$$
0 \geq w^{i j}\left[c^{k, \ell} c_{i j, k} c_{\ell, s t}-c_{i j, s t}\right] c^{s, p} c^{t, q} w_{p 1} w_{q 1}-C
$$

for some universal constant $C$. (When doing the computations, one has to remember that the derivatives of $\varphi$ depend on derivatives of $\nabla u$, or equivalently on derivatives of $T$.) By a rotation of coordinates that leaves the $e_{1}$ direction invariant 13 one can further assume that $\left(w_{i j}\right)$ is diagonal at $x_{0}$. We then obtain

$$
w^{i i}\left[c^{k, \ell} c_{i i, k} c_{\ell, s t}-c_{i i, s t}\right] c^{s, 1} c^{t, 1} w_{11} w_{11} \leq C .
$$

Up to now, the MTW condition has not been used.

We now apply $\operatorname{MTW}(K)$ to the vectors $\xi_{1}=\left(0, \sqrt{w^{22}}, \ldots, \sqrt{w^{n n}}\right)$ and $\xi_{2}=$ $\left(w_{11}, 0, \ldots, 0\right)$ to get

$$
K w_{11}^{2} \sum_{i=2}^{n} w^{i i} \leq C+w^{11}\left[c^{k, \ell} c_{11, k} c_{\ell, s t}-c_{11, s t}\right] c^{s, 1} c^{t, 1} w_{11} w_{11},
$$

which gives (using $(\mathbf{C 0})$ and the fact that $w^{11}=\left(w_{11}\right)^{-1}$ )

$$
w_{11}^{2} \sum_{i=2}^{n} w^{i i} \leq C\left(1+w_{11}\right) \text {. }
$$

\footnotetext{
${ }^{13}$ This is possible since $e_{1}$ is a maximum point for the map $\xi \mapsto \eta\left(x_{0}\right) w_{\xi \xi}$, therefore (except in the trivial case $\left.\eta\left(x_{0}\right)=0\right) w_{1 i}=0$ for every $i \neq 1$.
} 
Recalling that $w^{i j}=\left(w_{i j}\right)^{-1}$, by the arithmetic-geometric inequality and (4.5), we have

$$
\frac{1}{n-1} \sum_{i=2}^{n} w^{i i} \geq\left(\prod_{i=2}^{n} w^{i i}\right)^{1 /(n-1)} \geq c_{0}\left(w^{11}\right)^{-1 /(n-1)}=c_{0} w_{11}^{1 /(n-1)}
$$

where $c_{0}:=\inf _{x \in X} h(x, \nabla u(x))^{-1 /(n-1)}>0$. Hence, combining the above estimate with (4.11), we finally obtain

$$
\left[w_{11}\left(x_{0}\right)\right]^{2+1 /(n-1)} \leq C\left(1+w_{11}\left(x_{0}\right)\right)
$$

which proves that $G(x, \xi) \leq G\left(x_{0}, \xi_{0}\right) \leq C$ for all $(x, \xi) \in X \times \mathbb{S}^{n-1}$, as desired.

Remark 4.3. Apart from having its own interest, this general theory of MongeAmpère type equations satisfying the MTW condition turns out to be useful also in the classical case.

Indeed the MTW condition can be proven to be coordinate invariant $[73,84$, 87. This implies that if $u$ solves an equation of the form (1.2) with $\mathcal{A}$ satisfying the MTW condition (see (4.4) and $\Phi$ is a smooth diffeomorphism, then $u \circ \Phi$ satisfies an equation of the same form with a new matrix $\tilde{\mathcal{A}}$ which still satisfies the MTW condition. This means for instance that, if one wants to prove some boundary regularity result, through a smooth diffeomorphism one can reduce to a nicer situation. In particular this remark applies to the classical Monge-Ampère equation (which trivially satisfies $\operatorname{MTW}(0)$ ): if $u$ solves (1.1), then $u \circ \Phi$ satisfies (1.2) for some matrix $\mathcal{A}$ for which $\operatorname{MTW}(0)$ holds.

This observation is extremely natural in relation to the optimal transport problem: Consider for instance the optimal transport problem with cost $-x \cdot y$ between two smooth densities supported on smooth uniformly convex domains. By Theorem 3.3 we know that the optimal map $T$ is smooth. However, if one performs smooth change of coordinates $\Phi: X \rightarrow \Phi(X)$ and $\Psi: Y \rightarrow \Psi(Y)$, under these transformations the cost function becomes $-\Phi^{-1}(x) \cdot \Psi^{-1}(y)$ and the optimal transport map is given by $\Psi \circ T \circ \Phi^{-1}$, which is still smooth. However, this regularity of the optimal map cannot be deduced anymore from Theorem 3.3. while Theorem 4.2 still applies providing the expected regularity.

4.1. A geometric interpretation of the MTW condition. Although the MTW condition seemed the right assumption to obtain regularity of optimal maps, it was only after Loeper's work [84 that people started to have a good understanding of this condition, and a more geometric insight. Loeper's idea was the following: for the classical Monge-Ampère equation, a key property to prove regularity of convex solutions is that the subdifferential of a convex function is convex and so, in particular, connected. Roughly speaking, this has the following consequence: whenever a convex function $u$ is not $C^{1}$ at a point $x_{0}$, there is at least a whole segment contained in the subdifferential of $u$ at $x_{0}$, and this fact combined with the Monge-Ampère equation provides a contradiction. (See also Theorem 4.7 below.) Hence, Loeper wanted to understand whether the $c$-subdifferential of a $c$-convex function is at least connected (recall (3.9)), believing that this fact had a link with the regularity.

We wish to find some simple enough conditions implying the connectedness of sets $\partial_{c} \psi$. Of course if $\partial_{c} \psi(\bar{x})$ is a singleton, the connectedness is automatic, so we should look for nonsmooth functions. 
The easiest example is provided by the maximum of two cost functions: fix $y_{0}, y_{1} \in Y, a_{0}, a_{1} \in \mathbb{R}$, and define

$$
\psi(x):=\max \left\{-c\left(x, y_{0}\right)+a_{0},-c\left(x, y_{1}\right)+a_{1}\right\} .
$$

Take a point $\bar{x} \in\left\{x \mid-c\left(x, y_{0}\right)+a_{0}=-c\left(x, y_{1}\right)+a_{1}\right\}$, and let $\bar{y} \in \partial_{c} \psi(\bar{x})$. Since $\psi(x)+c(x, \bar{y})$ attains its minimum at $x=\bar{x}$, we get

$$
0 \in \partial_{\bar{x}}^{-}(\psi+c(\cdot, \bar{y}))
$$

(recall (3.10) for the definition of the Fréchet subdifferential) or equivalently

$$
-\nabla_{x} c(\bar{x}, \bar{y}) \in \partial^{-} \psi(\bar{x}) .
$$

From the above inclusion, one can easily deduce that $\bar{y} \in c-\exp _{\bar{x}}\left(\partial^{-} \psi(\bar{x})\right)$. Moreover, it is not difficult to see that

$$
\begin{aligned}
\partial^{-} \psi(\bar{x}) & =\left\{(1-t) v_{0}+t v_{1} \mid t \in[0,1]\right\}, \\
v_{i} & :=\nabla_{x} c\left(\bar{x}, y_{i}\right)=\left(c-\exp _{\bar{x}}\right)^{-1}\left(y_{i}\right), \quad i=0,1 .
\end{aligned}
$$

Therefore, denoting by $\left[v_{0}, v_{1}\right]$ the segment joining $v_{0}$ and $v_{1}$, we obtain

$$
\partial_{c} \psi(\bar{x}) \subset c-\exp _{\bar{x}}\left(\left[v_{0}, v_{1}\right]\right) .
$$

The above formula suggests the following definition:

Definition 4.4. Let $\bar{x} \in X, y_{0}, y_{1} \in Y$. Then we define the $c$-segment from $y_{0}$ to $y_{1}$ with base $\bar{x}$ as

$$
\left[y_{0}, y_{1}\right]_{\bar{x}}:=\left\{y_{t}=c-\exp _{\bar{x}}\left((1-t)\left(c-\exp _{\bar{x}}\right)^{-1}\left(y_{0}\right)+t\left(c-\exp _{\bar{x}}\right)^{-1}\left(y_{1}\right)\right) \mid t \in[0,1]\right\} .
$$

In [87, Ma, Trudinger, and Wang showed that, in analogy with the quadratic case (see Section [3.2), the convexity of $D_{x} c(x, Y)$ for all $x \in X$ is necessary for regularity. By slightly modifying their argument, Loeper 84 showed that the connectedness of the $c$-subdifferential is a necessary condition for the smoothness of optimal transport (see also [110, Theorem 12.7]):

Theorem 4.5. Assume that there exist $\bar{x} \in X$ and $\psi: X \rightarrow \mathbb{R}$ c-convex such that $\partial_{c} \psi(\bar{x})$ is not connected. Then one can construct smooth positive probability densities $f$ and $g$ such that the optimal map is discontinuous.

While the above result was essentially contained in [87, Loeper's major contribution was to link the connectedness of the $c$-subdifferential to a differential condition on the cost function, which (as we shall see later in Section 4.2) coincides with the MTW condition. In [84 he proved (a slightly weaker version of) the following result (see [110, Chapter 12] for a more general statement):

Theorem 4.6. The following conditions are equivalent:

(i) For any $\psi$ c-convex, for all $\bar{x} \in X, \partial_{c} \psi(\bar{x})$ is connected.

(ii) For any $\psi$ c-convex, for all $\bar{x} \in X,\left(c-\exp _{\bar{x}}\right)^{-1}\left(\partial_{c} \psi(\bar{x})\right)$ is convex, and it coincides with $\partial^{-} \psi(\bar{x})$.

(iii) For all $\bar{x} \in X$, for all $y_{0}, y_{1} \in Y$, if $\left[y_{0}, y_{1}\right]_{\bar{x}}=\left(y_{t}\right)_{t \in[0,1]} \subset Y$, then

$$
c\left(x, y_{t}\right)-c\left(\bar{x}, y_{t}\right) \geq \min \left[c\left(x, y_{0}\right)-c\left(\bar{x}, y_{0}\right), c\left(x, y_{1}\right)-c\left(\bar{x}, y_{1}\right)\right]
$$

for all $x \in X, t \in[0,1]$. 
(iv) For all $\bar{x} \in X, y \in Y, \eta, \xi \in \mathbb{R}^{n}$ with $\xi \perp \eta$,

$$
\begin{aligned}
& \left.\left.\quad \frac{d^{2}}{d s^{2}}\right|_{s=0} \frac{d^{2}}{d t^{2}}\right|_{t=0} c\left(c-\exp _{\bar{x}}(t \xi), c-\exp _{\bar{x}}(p+s \eta)\right) \leq 0, \\
& \text { where } p=\left(c-\exp _{\bar{x}}\right)^{-1}(y) .
\end{aligned}
$$

Moreover, if any of these conditions is not satisfied, $C^{1} c$-convex functions are not dense in the set of Lipschitz c-convex functions.

Sketch of the proof. We give here only some elements of the proof.

(ii) $\Rightarrow(i)$. Since $\left(c-\exp _{\bar{x}}\right)^{-1}\left(\partial_{c} \psi(\bar{x})\right)$ is convex, it is connected, and so its image by $c$ - $\exp _{\bar{x}}$ is connected too.

(i) $\Rightarrow$ (ii). For $\psi_{\bar{x}, y_{0}, y_{1}}:=\max \left\{-c\left(\cdot, y_{0}\right)+c\left(\bar{x}, y_{0}\right),-c\left(\cdot, y_{1}\right)+c\left(\bar{x}, y_{1}\right)\right\}$, we have

$$
\left(c-\exp _{\bar{x}}\right)^{-1}\left(\partial_{c} \psi_{\bar{x}, y_{0}, y_{1}}(\bar{x})\right) \subset\left[\left(c-\exp _{\bar{x}}\right)^{-1}\left(y_{0}\right),\left(c-\exp _{\bar{x}}\right)^{-1}\left(y_{1}\right)\right],
$$

which is a segment. Since in this case connectedness is equivalent to convexity, if (i) holds, we obtain $\partial_{c} \psi_{\bar{x}, y_{0}, y_{1}}(\bar{x})=\left[y_{0}, y_{1}\right]_{\bar{x}}=c-\exp _{\bar{x}}\left(\partial^{-} \psi_{\bar{x}, y_{0}, y_{1}}(\bar{x})\right)$.

In the general case, we fix $y_{0}, y_{1} \in \partial_{c} \psi(\bar{x})$. Then it is simple to see that

$$
\partial_{c} \psi(\bar{x}) \supset \partial_{c} \psi_{\bar{x}, y_{0}, y_{1}}(\bar{x})=\left[y_{0}, y_{1}\right]_{\bar{x}},
$$

and the result follows easily.

(ii) $\Leftrightarrow$ (iii). Condition (4.12) is equivalent to $\partial_{c} \psi_{\bar{x}, y_{0}, y_{1}}=\left[y_{0}, y_{1}\right]_{\bar{x}}$. Then the equivalence between (ii) and (iii) follows by arguing as above.

(iii) $\Rightarrow$ (iv). Fix $\bar{x} \in X$ and $y \in Y$ with $y=c-\exp _{\bar{x}}(p)$. Take $\xi, \eta$ orthogonal and with unit norm, and define

$$
y_{0}:=c-\exp _{\bar{x}}(p-\varepsilon \eta), \quad y_{1}:=c-\exp _{\bar{x}}(p+\varepsilon \eta) \quad \text { for some } \varepsilon>0 \text { small. }
$$

Moreover, let

$$
\begin{aligned}
h_{0}(x):=c\left(\bar{x}, y_{0}\right)-c\left(x, y_{0}\right), \quad h_{1}(x):=c\left(\bar{x}, y_{1}\right)-c\left(x, y_{1}\right), & \\
\psi & :=\max \left\{h_{0}, h_{1}\right\}=\psi_{\bar{x}, y_{0}, y_{1}} .
\end{aligned}
$$

We now define a curve $\gamma(t)$ contained in the set $\left\{h_{0}=h_{1}\right\}$ such that $\gamma(0)=\bar{x}$, $\dot{\gamma}(0)=\xi$.

Since $y \in\left[y_{0}, y_{1}\right]_{\bar{x}}$, by (iii) we get $y \in \partial_{c} \psi(\bar{x})$, so

$$
\begin{aligned}
\frac{1}{2}\left[h_{0}(\bar{x})+h_{1}(\bar{x})\right]+c(\bar{x}, y) & =\psi(\bar{x})+c(\bar{x}, y) \leq \psi(\gamma(t))+c(\gamma(t), y) \\
& =\frac{1}{2}\left[h_{0}(\gamma(t))+h_{1}(\gamma(t))\right]+c(\gamma(t), y),
\end{aligned}
$$

where we used that $h_{0}=h_{1}$ along $\gamma$. Recalling the definition of $h_{0}$ and $h_{1}$, we deduce that

$$
\frac{1}{2}\left[c\left(\gamma(t), y_{0}\right)+c\left(\gamma(t), y_{1}\right)\right]-c(\gamma(t), y) \leq \frac{1}{2}\left[c\left(\bar{x}, y_{0}\right)+c\left(\bar{x}, y_{1}\right)\right]-c(\bar{x}, y),
$$

so the function $t \mapsto \frac{1}{2}\left[c\left(\gamma(t), y_{0}\right)+c\left(\gamma(t), y_{1}\right)\right]-c(\gamma(t), y)$ achieves its maximum at $t=0$. This implies

i.e.,

$$
\left.\frac{d^{2}}{d t^{2}}\right|_{t=0}\left[\frac{1}{2}\left(c\left(\gamma(t), y_{0}\right)+c\left(\gamma(t), y_{1}\right)\right)-c(\gamma(t), y)\right] \leq 0
$$

$$
\left\langle\left[\frac{1}{2}\left(D_{x x} c\left(\bar{x}, y_{0}\right)+D_{x x} c\left(\bar{x}, y_{1}\right)\right)-D_{x}^{2} c(\bar{x}, y)\right] \cdot \xi, \xi\right\rangle \leq 0
$$


(here we used that $\left.\nabla_{x} c(\bar{x}, y)=\frac{1}{2}\left[\nabla_{x} c\left(\bar{x}, y_{0}\right)+\nabla_{x} c\left(\bar{x}, y_{1}\right)\right]\right)$. Thus the function

$$
s \mapsto\left\langle D_{x x} c\left(\bar{x}, c-\exp _{\bar{x}}(p+s \eta)\right) \cdot \xi, \xi\right\rangle
$$

is concave, proving (iv).

To understand why the above properties are related to smoothness, consider Theorem 4.6(iii). It says that if we take the function

$$
\psi_{\bar{x}, y_{0}, y_{1}}=\max \left\{-c\left(\cdot, y_{0}\right)+c\left(\bar{x}, y_{0}\right),-c\left(\cdot, y_{1}\right)+c\left(\bar{x}, y_{1}\right)\right\},
$$

then we are able to touch the graph of this function from below at $\bar{x}$ with the family of functions $\left\{-c\left(\cdot, y_{t}\right)+c\left(\bar{x}, y_{t}\right)\right\}_{t \in[0,1]}$. This suggests that we could use this family to regularize the cusp of $\psi_{\bar{x}, y_{0}, y_{1}}$ at the point $\bar{x}$, by slightly moving above the graphs of the functions $-c\left(\cdot, y_{t}\right)+c\left(\bar{x}, y_{t}\right)$. On the other hand, if (4.12) does not hold, it is not clear how to regularize the cusp preserving the condition of being $c$-convex.

By what we said above, the regularity property seems mandatory for developing a theory of smoothness of optimal transport. Indeed, if it is not satisfied, we can construct $C^{\infty}$ strictly positive densities $f, g$ such that the optimal map is not continuous. Hence the natural questions are when it is satisfied, and what is the link with the MTW condition.

Note also that the failure of the connectedness of $\partial_{c} u$ is a local obstruction to the regularity of optimal transport maps to be compared to the case of the quadratic cost where the obstruction to the regularity is the failure of the convexity of $Y$, a global condition.

4.2. Regularity results. As we have seen in Theorem 4.6, roughly speaking the connectedness of the $c$-subdifferential of $c$-convex functions is equivalent to

$$
\left.\left.\frac{d^{2}}{d s^{2}}\right|_{s=0} \frac{d^{2}}{d t^{2}}\right|_{t=0} c\left(c-\exp _{x}(t \xi), c-\exp _{x}(p+s \eta)\right) \leq 0,
$$

for all $p, \xi, \eta \in \mathbb{R}^{n}$, with $\xi$ and $\eta$ orthogonal, $p=\left(c-\exp _{x}\right)^{-1}(y)$. (This equivalence is not exact, since to show that (4.13) implies the connectedness of the $c$-subdifferential of $c$-convex functions, one needs to know that the target is $c$-convex to ensure that it contains the $c$-segment $\left[y_{0}, y_{1}\right]_{\bar{x}}$; see (iii) in Theorem 4.6 above.)

By some standard but tedious computations it is not difficult to check that the expression in (4.13) coincides up to the sign with the MTW tensor, i.e.,

$$
\begin{aligned}
\left.\left.\frac{d^{2}}{d s^{2}}\right|_{s=0} \frac{d^{2}}{d t^{2}}\right|_{t=0} c\left(c-\exp _{x}(t \xi), c-\exp _{x}(p+s \eta)\right) \\
\quad=\sum_{i, j, k, l, p, q, r, s}\left(c_{i j, r s}-c_{i j, p} c^{p, q} c_{q, r s}\right) c^{r, k} c^{s, l} \xi^{i} \xi^{j} \eta^{k} \eta^{l},
\end{aligned}
$$

where the derivatives of $c$ are evaluated at $(x, c-\exp (p))$. Hence

$$
\operatorname{MTW}(0) \Leftrightarrow \quad \Leftrightarrow \quad 4.13) \quad \Leftrightarrow \quad \partial_{c} \psi(x) \text { connected } \forall x, \psi c \text {-convex, }
$$

and by Theorems 4.2 and 4.5 the MTW condition is necessary and sufficient for the smoothness of the optimal transport map.

By exploiting (a variant of) Theorem 4.6, Loeper 84] proved the following regularity result (recall the notation in Theorem [3.6): 
Theorem 4.7. Let $c: X \times Y \rightarrow \mathbb{R}$ satisfy (C0)-(C3). Assume that the $\operatorname{MTW}(K)$ holds for some $K>0, f$ is bounded from above on $X, g$ is bounded away from zero on $Y$, and denote by $T$ the optimal transport map sending $f$ onto $g$. Finally, suppose that $D_{x} c(x, Y)$ is convex for any $x \in X$. Then $u \in C^{1, \alpha}(\bar{X})$, with $\alpha=1 /(4 n-1)$ (hence $T_{u} \in C^{0, \alpha}(\bar{X})$ ).

In a subsequent paper, Liu 80 proved the above result with $\alpha=1 /(2 n-1)$ and showed that such an exponent is optimal.

We notice however that this result does not include Caffarelli's result since the cost $-x \cdot y$ satisfies MTW(0). Hence, it would be nice to have a Hölder regularity theorem which includes at the same time Caffarelli's and Loeper's results.

As proved by the second author together with Kim and McCann [52, such a result is indeed true (see also [54,63, 113):

Theorem 4.8. Let $c: X \times Y \rightarrow \mathbb{R}$ satisfy (C0)-(C3). Assume that $\mathrm{MTW}(0)$ holds, $f$ is bounded from above on $X$, and $g$ is bounded away both from zero and infinity on $Y$. Also, suppose that $D_{x} c(x, Y)$ and $D_{y} c(Y, y)$ are uniformly convex for any $x \in X$ and $y \in Y$. Then $u \in C_{\mathrm{loc}}^{1, \alpha}\left(\overline{X^{\prime}}\right)$ for any open set $X^{\prime} \subset X$ where $f$ is uniformly bounded away from zero.

Let us mention that further extensions of Theorem 3.3 to this general setting have been obtained in $37,81,82$.

Note that Theorem 4.8 as well as the other results in $[37,63,81,82,113$, only deal with the interior regularity for optimal transport maps. It would be interesting to develop a boundary regularity theory, in the spirit of [20, 22], for the class of equations (1.2) arising in optimal transport.

We conclude this section by providing some examples of cost functions satisfying the MTW condition (where defined) and referring to [87] for more examples:

- $c_{1}(x, y)=-\log |x-y|$;

- $c_{2}(x, y)=\sqrt{a^{2}-|x-y|^{2}}$

- $c_{3}(x, y)=\sqrt{a^{2}+|x-y|^{2}}$;

- $c_{4}(x, y)=|x-y|^{p}$ with $-2<p<1$.

Note that the last two costs provide (respectively as $a \rightarrow 0^{+}$and $p \rightarrow 1^{-}$) an approximation of the Monge cost $c(x, y)=|x-y|$. Despite the fact that this latter cost does not satisfy any of the conditions $(\mathbf{C 0})-(\mathbf{C 3})$, existence of optimal transport maps can still be proved (see for instance [6] for an account of the theory) and some regularity results have been obtained in 60. Very recently, the strategy to approximate the Monge cost with $c_{3}$ has been used in [79] to deduce some estimates for the Monge problem by proving a priori bounds on the transport maps which are uniform as $a \rightarrow 0$.

4.3. The case of Riemannian manifolds. Let us consider the case when $c=$ $d^{2} / 2$ on $X=Y=(M, g)$ a compact Riemannian manifold without boundary. As we have seen in the previous section, the MTW condition and some suitable convexity of the domains are necessary to obtain regularity of optimal maps. Here we have also to face an additional problem. Indeed, while before the cost function was assumed to be smooth on $X \times Y$, in the case of a compact Riemannian manifold without boundary the function $d^{2} / 2$ is never smooth on the whole $M \times M$ due to the presence of the cut-locus (see Section 4.4 below for a more precise description). 
Before focusing on all these issues, let us start with the following example which will allow us to look at the MTW condition from a different perspective.

Example 4.9. We want to show how negative sectional curvature is an obstruction to regularity (indeed even to continuity) of optimal maps when $c=d^{2} / 2$. (We refer to [110, Theorem 12.4] for more details on the construction given below.)

Let $M=\mathbb{H}^{2}$ be the hyperbolic plane (or a compact quotient thereof). Fix a point $O$ as the origin, and fix a local system of coordinates in a neighborhood of $O$ such that the maps $\left(x_{1}, x_{2}\right) \mapsto\left( \pm x_{1}, \pm x_{2}\right)$ are local isometries (it suffices for instance to consider the model of the Poincare disk, with $O$ equal to the origin in $\mathbb{R}^{2}$ ). Then define the points

$$
A^{ \pm}=(0, \pm \varepsilon), \quad B^{ \pm}=( \pm \varepsilon, 0) \quad \text { for some } \varepsilon>0 .
$$

Take a measure $\mu$ symmetric with respect to 0 and concentrated near $\left\{A^{+}\right\} \cup\left\{A^{-}\right\}$ (say $3 / 4$ of the total mass belongs to a small neighborhood of $\left\{A^{+}\right\} \cup\left\{A^{-}\right\}$) and a measure $\nu$ symmetric with respect to 0 and concentrated near $\left\{B^{+}\right\} \cup\left\{B^{-}\right\}$. Moreover assume that $\mu$ and $\nu$ are absolutely continuous, and have positive smooth densities. We denote by $T$ the unique optimal transport map, and we assume by contradiction that $T$ is continuous. By symmetry we deduce that $T(O)=O$, and by counting the total mass there exists a point $A^{\prime}$ close to $A^{+}$which is sent to a point $B^{\prime}$ near, say, $B^{+}$.

But, by negative curvature (if $A^{\prime}$ and $B^{\prime}$ are close enough to $A$ and $B$, respectively), the Pythagoras Theorem becomes an inequality: $d\left(O, A^{\prime}\right)^{2}+d\left(O, B^{\prime}\right)^{2}<$ $d\left(A^{\prime}, B^{\prime}\right)^{2}$, and this contradicts the optimality of the transport map, as transporting $A^{\prime}$ onto $O$ and $O$ onto $B^{\prime}$ would be more convenient than transporting $A^{\prime}$ onto $B^{\prime}$ and letting $O$ stay at rest.

Now, the natural question is, How does the above example fit into Ma, Trudinger, and Wang and Loeper's results? The answer was given by Loeper [84] where he noticed that, when $c=d^{2} / 2$ on a Riemannian manifold $M$, the MTW tensor satisfies the following remarkable identity:

$$
\mathfrak{S}_{(x, x)}(\xi, \eta)=\frac{2}{3} \operatorname{Sect}_{x}([\xi, \eta]),
$$

where $\xi, \eta \in T_{x} M$ are two orthogonal unit vectors, and $\operatorname{Sect}_{x}([\xi, \eta])$ denotes the sectional curvature of the plane generated by $\xi$ and $\eta$.

Combining (4.14) with Theorems 4.5 and 4.6. we get the following important negative result:

Theorem 4.10. Let $(M, g)$ be a (compact) Riemannian manifold, and assume there exist $x \in M$ and a plane $P \subset T_{x} M$ such that $\operatorname{Sect}_{x}(P)<0$. Then there exist smooth positive probability densities $f$ and $g$ such that the optimal map for the cost $c=d^{2} / 2$ is discontinuous.

After this negative result, one could still hope to develop a regularity theory on any manifold with nonnegative sectional curvature. But this is not the case: as shown by Kim [72] (see also [56]) the regularity condition is strictly stronger than the condition of nonnegativity of sectional curvatures. In conclusion, except for some special cases, the optimal map is nonsmooth. 
Some examples of manifolds satisfying the MTW condition have been found in 55, 57, 73, 74, 84, 85,

- $\mathbb{R}^{n}$ and $\mathbb{T}^{n}$ satisfy $\operatorname{MTW}(0)$.

- $\mathbb{S}^{n}$, its quotients (like $\mathbb{R} \mathbb{P}^{n}$ ), and its submersions (like $\mathbb{C P}^{n}$ or $\mathbb{H} \mathbb{P}^{n}$ ), satisfy MTW(1).

- Products of any of the examples listed above (for instance, $\mathbb{S}^{n_{1}} \times \cdots \times \mathbb{S}^{n_{k}} \times$ $\mathbb{R}^{\ell}$ or $\left.\mathbb{S}^{n_{1}} \times \mathbb{C P}^{n_{2}} \times \mathbb{T}^{n_{3}}\right)$ satisfy $\operatorname{MTW}(0)$.

- Smooth perturbations of $\mathbb{S}^{n}$ satisfy $\operatorname{MTW}(K)$ for some $K>0$.

Now, in order to prove regularity of optimal transport maps on manifolds satisfying MTW, one would like to localize Theorems 4.7 and 4.8. However, as we already mentioned above, one has to face a very nontrivial issue: the cost function $d^{2} / 2$ is not everywhere smooth due to the presence of the cut-locus; hence, to localize those theorems, one would like to know the validity of the so-called "stay-away property",

$$
T(x) \notin \operatorname{cut}(x) \quad \forall x \in M .
$$

(See the next section for a precise definition of $\operatorname{cut}(x)$.) While this property has been proven to hold true in some special cases [41,42,53, it is still unknown in general and this creates several difficulties in the proof of regularity of optimal maps. In particular this is one of the reasons why, on perturbations of $\mathbb{S}^{n}$, only continuity (and not higher regularity) of optimal maps is currently known [57.

Another issue is the convexity of the target: in the case of compact manifolds without boundary, the assumption that $-D_{x} c(x, Y)$ is convex for every $x \in X$ (see for instance Theorem 4.7) corresponds to the convexity of the cut-locus of the manifold when seen from any tangent space. This shows that regularity of solutions is strongly related to the convexity of the cut-locus. Actually, as we shall describe now, the MTW condition has even much stronger links with the cut-locus.

4.4. MTW vs. cut-locus. To explain the connection between the MTW condition and the convexity of the cut-locus, we start by recalling some basic definitions.

Given a tangent vector $v \in T_{x} M$, the curve $\left(\exp _{x}(t v)\right)_{t \geq 0}$ is a geodesic defined for all times, but in general is not minimizing for large times. On the other hand, it is possible to prove that $\exp _{x}(t v)$ is always minimizing between $x$ and $\exp _{x}(\varepsilon v)$ for $\varepsilon>0$ sufficiently small. We define the cut-time $t_{C}(x, v)$ as

$t_{C}(x, v):=\inf \left\{t>0: s \mapsto \exp _{x}(s v)\right.$ is not minimizing between $x$ and $\left.\exp _{x}(t v)\right\}$.

Given $x \in M$, we define the cut-locus of $x$

$$
\operatorname{cut}(x):=\left\{\exp _{x}\left(t_{C}(x, v) v\right): v \in T_{x} M,|v|_{x}=1\right\},
$$

the tangent cut-locus of $x$

$$
\operatorname{TCL}(x):=\left\{t_{C}(x, v) v: v \in T_{x} M,|v|_{x}=1\right\},
$$

and the injectivity domain of the exponential map at $x$

$$
\mathrm{I}(x):=\left\{t v: 0 \leq t<t_{C}(x, v), v \in T_{x} M,|v|_{x}=1\right\} .
$$

With these definitions, we have

$$
\operatorname{cut}(x)=\exp _{x}(\mathrm{TCL}(x)), \quad \operatorname{TCL}(x)=\partial(\mathrm{I}(x)) .
$$

For instance, on the sphere $\mathbb{S}^{n}, t_{C}(x, v)=\pi /|v|_{x}, \operatorname{cut}(x)=\{-x\}, \mathrm{I}(x)=B_{\pi}(0)$, and $\operatorname{TCL}(x)=\partial B_{\pi}(0)$. 
It is possible to prove that, if $y \notin \operatorname{cut}(x)$, then $x$ and $y$ are joined by a unique minimizing geodesic. The converse is close to being true: $y \notin \operatorname{cut}(x)$ if and only if there are neighborhoods $U$ of $x$ and $V$ of $y$ such that any two points $x^{\prime} \in U$ and $y^{\prime} \in V$ are joined by a unique minimizing geodesic. In particular $y \notin \operatorname{cut}(x)$ if and only if $x \notin \operatorname{cut}(y)$.

The fact that a point $y \in M$ belongs to $\operatorname{cut}(x)$ is a phenomenon which is captured by the regularity of the distance function. Indeed, it can be proven that the following holds (see for instance [30, Proposition 2.5]):

(a) The function $d(x, \cdot)^{2}$ is smooth in a neighborhood of $y$ if and only if $y \notin$ $\operatorname{cut}(x)$.

(b) The function $d(x, \cdot)^{2}$ has an upward Lipschitz cusp at $y$ if and only if $y \in \operatorname{cut}(x)$ and there are at least two minimizing geodesics between $x$ and $y$.

(c) The function $d(x, \cdot)^{2}$ is $C^{1}$ at $y$ and its Hessian has an eigenvalue $-\infty$ if and only if $y \in \operatorname{cut}(x)$ and there is a unique minimizing geodesics between $x$ and $y$.

In the above statement, having an upward cusp means that there exist two vectors $p_{1} \neq p_{2}$ both belonging to the supergradient of $f:=d(x, \cdot)^{2}$ at $y$ : writing everything in charts, we have

$$
\left\{p_{1}, p_{2}\right\} \subset \partial^{+} f(y):=\{p: f(y+v) \leq f(y)+\langle p, v\rangle+o(|v|) \quad \forall v\},
$$

that is $f$ is locally below the function $v \mapsto f(y)+\min \left\{\left\langle p_{1}, v\right\rangle,\left\langle p_{2}, v\right\rangle\right\}+o(|v|)$ near $y$. Hence (b) corresponds roughly to saying that the second derivative (along the direction $p_{2}-p_{1}$ ) of $d(x, \cdot)^{2}$ at $y$ is $-\infty$. (The fact that there is an upward cusp means that one of the second directional derivatives is a negative delta measure.)

Furthermore, saying that the "Hessian has an eigenvalue $-\infty$ " means that (always working in charts)

$$
\liminf _{|v| \rightarrow 0} \frac{f(y+v)-2 f(y)+f(y-v)}{|v|^{2}}=-\infty .
$$

Thus, the above description of the cut-locus in terms of the squared distance can be informally summarized as

$$
y \in \operatorname{cut}(x) \quad \Leftrightarrow \quad D_{y y} d^{2}(x, y) v \cdot v=-\infty \quad \text { for some } v \in T_{y} M .
$$

Based on this observation, in [86] Loeper and Villani noticed the existence of a deep connection between the MTW condition and the geometry of the cut-locus. The idea is the following: Fix $x \in M$, and let $v_{0}, v_{1} \in \mathrm{I}(x)$. Consider the segment $\left(v_{t}\right)_{t \in[0,1]}$, with $v_{t}:=(1-t) v_{0}+t v_{1}$. Set further $y_{t}:=\exp _{x}\left(v_{t}\right)$. Since $v_{0}, v_{1} \in \mathrm{I}(x)$ we have

$$
y_{0}, y_{1} \notin \operatorname{cut}(x) \text {. }
$$

In particular $c(x, \cdot):=d(x, \cdot)^{2} / 2$ is smooth in a neighborhood of $y_{0}$ and $y_{1}$. Assume now that $\operatorname{MTW}(0)$ holds. Thanks to Theorem 4.6(iv), we know that the function

$$
\eta \mapsto\left\langle D_{x x} c\left(\bar{x}, \exp _{\bar{x}}(p+\eta)\right) \cdot \xi, \xi\right\rangle
$$

is concave for all $\eta \perp \xi$. (This is just a formal argument, as the theorem applies a priori only if $\exp _{\bar{x}}(p+\eta) \notin \operatorname{cut}(\bar{x})$.) Applying this fact along the segment $\left(v_{t}\right)_{t \in[0,1]}$ and exploiting the smoothness of $d(x, \cdot)^{2}$ near $y_{0}$ and $y_{1}$, for $\xi \perp\left(v_{1}-v_{0}\right)$, we obtain

$$
\inf _{t \in[0,1]}\left\langle D_{x x} d^{2}\left(x, y_{t}\right) \cdot \xi, \xi\right\rangle \geq \min \left\{\left\langle D_{x x} d^{2}\left(x, y_{0}\right) \cdot \xi, \xi\right\rangle,\left\langle D_{x x} d^{2}\left(x, y_{1}\right) \cdot \xi, \xi\right\rangle\right\} \geq C_{0},
$$


for some constant $C_{0} \in \mathbb{R}$. Hence, if we forget for a moment about the orthogonality assumption between $v_{1}-v_{0}$ and $\xi$, we see that the above equation implies that $x \notin \operatorname{cut}\left(y_{t}\right)$ for all $t \in[0,1]$ (compare with (4.16) ), which by symmetry gives

$$
y_{t} \notin \operatorname{cut}(x) \quad \forall t \in[0,1] \text {, }
$$

or equivalently

$$
v_{t} \notin \operatorname{TCL}(x) \quad \forall t \in[0,1] .
$$

Since $v_{0}, v_{1} \in \mathrm{I}(x)$, we have obtained

$$
v_{t} \in \mathrm{I}(x) \quad \forall t \in[0,1],
$$

that is $\mathrm{I}(x)$ is convex. In conclusion, this formal argument suggests that the MTW condition (or a variant of it) should imply that all tangent injectivity domains $\mathrm{I}(x)$ are convex, for every $x \in M$.

This would be a remarkable property. Indeed, usually the only regularity results available for $\mathrm{I}(x)$ say that $\mathrm{TCL}(x)$ is just Lipschitz [67. Moreover, such a result would be of a global nature and not just local like a semiconvexity property. Unfortunately, the argument described above is just formal and up to now there is no complete result in that direction. Still, one can prove some rigorous results in some special cases [55, 58, 86.

As we mentioned before, the convexity of the tangent cut-loci is not only an interesting geometric property but it is also important for the regularity of optimal transport maps, since it corresponds to the assumption that $-D_{x} c(x, Y)$ is convex for every $x \in X$. Indeed, as shown in [58, both the MTW condition and the convexity of the tangent cut-loci are necessary (and in some cases sufficient) conditions for the continuity of optimal transport maps on manifolds.

4.5. Partial regularity. In the case of the classical Monge-Ampère equation, we saw that convexity of the target is a necessary condition for the regularity of Brenier solutions to the Monge-Ampère equation. Now, in the case of a general cost, in addition to some suitable convexity of the target, one needs to assume the validity of the MTW condition. This condition however is known to be true only for few examples of costs (for instance, all costs of the form $|x-y|^{p}$ with $p \notin(-2,1) \cup\{2\}$ do not satisfy the MTW condition, see however 23. for some "perturbative" results in this case). Moreover, only a few manifolds are known to satisfy it (essentially just the ones listed in Section 4.3), and for instance it is known to fail on sufficiently flat ellipsoids [56].

Let us also recall that, in the case of a Riemannian manifold, the MTW condition implies that the sectional curvature is nonnegative at every point (see (4.14)). Therefore, if we consider a compact manifold $(M, g)$ with negative sectional curvature, we have that the MTW condition fails at every point. This fact could make one suspect that the transport map could be extremely irregular. However, as shown by the authors in [39], this negative picture cannot happen:

Theorem 4.11. Let $X, Y \subset \mathbb{R}^{n}$ be two bounded open sets, and let $f: X \rightarrow \mathbb{R}^{+}$ and $g: Y \rightarrow \mathbb{R}^{+}$be two continuous probability densities, respectively, bounded away from zero and infinity on $X$ and $Y$. Assume that the cost $c: X \times Y \rightarrow \mathbb{R}$ satisfies (C0)-(C3), and denote by $T: X \rightarrow Y$ the unique optimal transport map sending $f$ onto $g$. Then there exist two relatively closed sets $\Sigma_{X} \subset X, \Sigma_{Y} \subset Y$ of measure zero such that $T: X \backslash \Sigma_{X} \rightarrow Y \backslash \Sigma_{Y}$ is a homeomorphism of class $C_{\text {loc }}^{0, \beta}$ for any $\beta<1$. In addition, if $c \in C_{\mathrm{loc}}^{k+2, \alpha}(X \times Y), f \in C_{\mathrm{loc}}^{k, \alpha}(X)$, and $g \in C_{\mathrm{loc}}^{k, \alpha}(Y)$ for 
some $k \geq 0$ and $\alpha \in(0,1)$, then $T: X \backslash \Sigma_{X} \rightarrow Y \backslash \Sigma_{Y}$ is a diffeomorphism of class $C_{\text {loc }}^{k+1, \alpha}$.

By suitably localizing this result, in [39] we could also prove the following:

Theorem 4.12. Let $M$ be a smooth Riemannian manifold, and let $f, g: M \rightarrow \mathbb{R}^{+}$ be two continuous probability densities, locally bounded away from zero and infinity on $M$. Let $T: M \rightarrow M$ denote the optimal transport map for the cost $c=d^{2} / 2$ sending $f$ onto $g$. Then there exist two closed sets $\Sigma_{X}, \Sigma_{Y} \subset M$ of measure zero such that $T: M \backslash \Sigma_{X} \rightarrow M \backslash \Sigma_{Y}$ is a homeomorphism of class $C_{\mathrm{loc}}^{0, \beta}$ for any $\beta<1$. In addition, if both $f$ and $g$ are of class $C^{k, \alpha}$, then $T: M \backslash \Sigma_{X} \rightarrow M \backslash \Sigma_{Y}$ is a diffeomorphism of class $C_{\mathrm{loc}}^{k+1, \alpha}$.

As discussed before, when the MTW condition fails, the obstruction to the regularity of optimal maps is local, while in the quadratic case it was a global obstruction (namely the nonconvexity of the target which does not allow us to have good control on the Monge-Ampère measure of $u$ ). In the quadratic cost case, in [49,51] it was shown that on a big set there is still good control on the Monge-Ampère measure of the potential $u$ allowing us to apply the local theory of classical Monge-Ampère equation; see the sketch of the proof of Theorem 3.4. However, the failure of the MTW condition does not allow us to use any local regularity estimate for the PDE, therefore a completely new strategy with respect to [49, 51] has to be used.

The rough idea is the following: First of all recall that, thanks to Theorem 3.6. the optimal transport map $T$ is of the form $T_{u}(x)=c$-exp $x(\nabla u(x))$ for some $c$ convex function $u$. Then, if $\bar{x}$ is a point where $u$ is twice differentiable (see Remark 3.5), around that point $u$ looks like a parabola. In addition, by looking close enough to $\bar{x}$, the cost function $c$ will be very close to the linear one and the densities will be almost constant there. Hence $u$ is close to a convex function $v$ solving an optimal transport problem with linear cost and constant densities. In addition, since $u$ is close to a parabola, so is $v$. Hence, by Caffarelli's regularity theory, $v$ is smooth and we can use this information to deduce that $u$ is even closer to a second parabola (given by the second-order Taylor expansion of $v$ at $\bar{x}$ ) inside a small neighborhood. By rescaling back this neighborhood at scale 1 and iterating this construction, we obtain that $u$ is $C^{1, \beta}$ at $\bar{x}$ for every $\beta \in(0,1)$. Since this argument can be applied at every point in a neighborhood of $\bar{x}$, we deduce that $u$ is $C^{1, \beta}$ there. This is summarized in the following

Proposition 4.13. Let $\mathcal{C}_{1}$ and $\mathcal{C}_{2}$ be two closed sets satisfying

$$
B_{1 / 3} \subset \mathcal{C}_{1}, \mathcal{C}_{2} \subset B_{3},
$$

let $f, g$ be two densities supported in $\mathcal{C}_{1}$ and $\mathcal{C}_{2}$, respectively, and let $u: \mathcal{C}_{1} \rightarrow \mathbb{R}$ be a c-convex function such that $\partial_{c} u\left(\mathcal{C}_{1}\right) \subset B_{3}$ and $\left(T_{u}\right)_{\sharp}(f d x)=g d y$. Then, for every $\beta \in(0,1)$ there exists a constant $\eta_{0}>0$ such that the following holds. If

$$
\left\|f-\mathbf{1}_{\mathcal{C}_{1}}\right\|_{\infty}+\left\|g-\mathbf{1}_{\mathcal{C}_{2}}\right\|_{\infty}+\|c(x, y)+x \cdot y\|_{C^{2}\left(B_{3} \times B_{3}\right)}+\left\|u-\frac{1}{2}|x|^{2}\right\|_{C^{0}\left(B_{3}\right)} \leq \eta_{0},
$$

then $u \in C^{1, \beta}\left(B_{1 / 6}\right)$.

Once this result is proved, we know that $\partial^{-} u$ is a singleton at every point, so it follows from (3.13) that

$$
\partial_{c} u(x)=c-\exp _{x}\left(\partial^{-} u(x)\right) .
$$


(The above identity is exactly what in general may fail for general $c$-convex functions unless the MTW condition holds.) Thanks to this fact we obtain that $u$ enjoys a comparison principle, and this allows us to use a second approximation argument with solutions of the classical Monge-Ampère equation (in the spirit of [22]) to conclude that $u$ is $C^{2, \sigma^{\prime}}$ in a smaller neighborhood, for some $\sigma^{\prime}>0$. Then higher regularity follows from standard elliptic estimates.

Proposition 4.14. Let $u, f, g$ be as in Proposition 4.13, and assume in addition that $c \in C^{k, \alpha}\left(B_{3} \times B_{3}\right)$ and $f, g \in C^{k, \alpha}\left(B_{1 / 3}\right)$ for some $k \geq 0$ and $\alpha \in(0,1)$. There exists a small constant $\eta_{1}$ such that if

$$
\left\|f-\mathbf{1}_{\mathcal{C}_{1}}\right\|_{\infty}+\left\|g-\mathbf{1}_{\mathcal{C}_{2}}\right\|_{\infty}+\|c(x, y)+x \cdot y\|_{C^{2}\left(B_{3} \times B_{3}\right)}+\left\|u-\frac{1}{2}|x|^{2}\right\|_{C^{0}\left(B_{3}\right)} \leq \eta_{1},
$$

then $u \in C^{k+2, \alpha}\left(B_{1 / 6}\right)$.

These results imply that $T_{u}$ is of class $C^{0, \beta}$ in neighborhood of $\bar{x}$ (resp. $T_{u}$ is of class $C^{k+1, \alpha}$ if $c \in C_{\mathrm{loc}}^{k+2, \alpha}$ and $f, g \in C_{\mathrm{loc}}^{k, \alpha}$ ). Being that our assumptions are completely symmetric in $x$ and $y$, we can apply the same argument to the optimal map $T^{*}$ sending $g$ onto $f$. Since $T^{*}=\left(T_{u}\right)^{-1}$, it follows that $T_{u}$ is a global homeomorphism of class $C_{\text {loc }}^{0, \beta}$ (resp. $T_{u}$ is a global diffeomorphism of class $C_{\text {loc }}^{k+1, \alpha}$ ) outside a closed set of measure zero.

\section{Open PROBlems AND FURTHER PERSPECTIVES}

In this last section we briefly describe some further material related to MongeAmpère type equations and state some open problems.

5.1. General prescribed Jacobian equations. Equations (3.4) and (4.3) can be seen as particular cases of prescribed Jacobian equations of the form

$$
\operatorname{det}(\nabla[T(x, u, \nabla u)])=\psi(x, u, \nabla u),
$$

where $T=T(x, z, p): \Omega \times \mathbb{R} \times \mathbb{R}^{n} \rightarrow \mathbb{R}^{n}$. In case $\operatorname{det}\left(\nabla_{p} T\right) \neq 0$, arguing as in Section 4 one sees that (5.1) becomes

$$
\operatorname{det}\left(D^{2} u-\mathcal{A}(x, u, \nabla u)\right)=f(x, u, \nabla u) .
$$

Let us notice that the classical Monge-Ampère equation corresponds to the case $T(x, z, p)=p$, and more generally the optimal transport case described in this note corresponds to the case

$$
\nabla_{x} c(x, T(x, p))=-p,
$$

that is $T(x, p)=c-\exp _{x}(p)$ (compare with Section 3.4).

Motivated by problems arising in geometric optics, Trudinger began in [101, 102 . a systematic study of equations of the form (5.1) - (5.2) in the particular case when $T$ is obtained through a generating function $G: \mathbb{R}^{n} \times \mathbb{R}^{n} \times \mathbb{R}$. Let us briefly present this theory, referring to 102 for more details.

Given $G$ as above, a function $u$ is said to be $G$-convex on $\Omega$ if for every $x_{0} \in \Omega$ there exist $y_{0}$ and $z_{0}$ such that

$$
u\left(x_{0}\right)=-G\left(x_{0}, y_{0}, z_{0}\right) \text { and } u(x) \geq-G\left(x, y_{0}, z_{0}\right) \quad \forall x \in \Omega .
$$

Then, under suitable assumptions on $G$, one can define the maps $T=T(x, z, p)$ and $Z=Z(x, z, p)$ through the relations

$$
\nabla_{x} G(x, T(x, u, p), Z(x, u, p))=-p, \quad-G(x, T(x, u, p), Z(x, u, p))=u .
$$


With this choice one gets from (5.3) that $y_{0}=T\left(x_{0}, u\left(x_{0}\right), D u\left(x_{0}\right)\right)$ whenever $u \in C^{1}$. Note that the optimal transportation case corresponds to the choice $G(x, y, z):=c(x, y)-z$.

Then, under some necessary structural conditions on $G$ (which are the analogue of the MTW condition in this context), in 101,102 Trudinger started developing a theory parallel to the one described in Section 4. It would be interesting to extend all the results valid in the optimal transportation case to this general setting. Also, in case $G$ does not satisfy such necessary structural conditions, it would be nice to check whether an analogue of Theorem 4.11 still holds.

5.2. Open problems. We list some open problems related to optimal transportation and the regularity theory for general Monge-Ampère type equations.

(1) As we already mentioned, in dimension 2 stronger regularity results for solutions of (1.1) are available [3, 21,54]. In particular, Alexandrov showed in [3] that $u$ is continuous differentiable assuming only the upper bound $\operatorname{det} D^{2} u \leq \lambda$. Hence, in relation to Theorem 2.22 a natural question becomes: is it possible to prove $W_{\text {loc }}^{2,1}$ (or even $W_{\text {loc }}^{2,1+\varepsilon}$ ) regularity of $u$ in the 2-d case assuming only an upper bound on $\operatorname{det} D^{2} u$ ? Apart from its own interest, such a result would have applications in relation to extending Theorem 2.24 outside of the periodic setting.

(2) In $\mathbb{R}^{2}$ there is a link between the classical Monge-Ampère equation (1.1) and the theory of quasi-conformal maps. Indeed, if $u$ is a smooth solution of (1.1), we can consider consider the maps $\Phi_{1}, \Phi_{2}: \Omega \rightarrow \mathbb{R}^{2}$ defined as

$$
\Phi_{1}(x, y):=\left(\partial_{x} u(x, y), y\right), \quad \Phi_{2}(x, y):=\left(x, \partial_{y} u(x, y)\right) .
$$

Then, if $\lambda \leq \operatorname{det} D^{2} u \leq 1 / \lambda$, a simple computation shows that $\Phi:=\Phi_{1} \circ$ $\left(\Phi_{2}\right)^{-1}$ is $1 / \lambda^{2}$-quasi-conformal, that is

$$
\lambda^{2}\|\nabla \Phi\|_{H S}^{2} \leq 2 \operatorname{det} \nabla \Phi
$$

where $\|\cdot\|_{H S}$ is the Hilbert-Schmidt norm. In view of the known higherintegrability theory for quasi-conformal maps [11, Theorem 13.2.1], one is led to conjecture that in dimension 2 the sharp version of Theorem 2.22 should be

$$
D^{2} u \in L_{w, \text { loc }}^{\frac{1+\lambda^{2}}{1-\lambda^{2}}}(\Omega) \quad \text { provided } \lambda \leq \operatorname{det} D^{2} u \leq 1 / \lambda \text { inside } \Omega,
$$

that is

$$
\sup _{s>0} s^{\frac{1+\lambda^{2}}{1-\lambda^{2}}}\left|\left\{\left|D^{2} u\right|>s\right\} \cap \Omega^{\prime}\right| \leq C \quad \forall \Omega^{\prime} \Subset \Omega .
$$

In particular one would get that $D^{2} u \in L_{\text {loc }}^{p}(\Omega)$ for all $p<\frac{1+\lambda^{2}}{1-\lambda^{2}}$.

(3) As we explained at the end of Section 2.5, in the case of smooth initial data, existence of smooth solutions to the semigeostrophic equations has been known only for a short time. In analogy with the 2-d incompressible Euler equations (see for instance [83. for a discussion on the analogy between these two equations), it would be extremely interesting to understand whether, at least in the 2 -d periodic case, there are conditions on the initial data which ensure global-in-time existence of smooth solutions. 
(4) The proof of the fact that the MTW condition holds on perturbations of the round sphere is extremely delicate and relies on the fact that, in the expression of the MTW tensor (4.4), 15 terms "magically" combine to form a "perfect square" (see [57, page 127]), allowing the authors to prove that the MTW tensor satisfies the right inequality. It seems unlikely to us that this is just a coincidence, and we believe that this fact should be a sign of the presence of an underlying and deeper structure which has not been yet found 14

(5) As mentioned in Section 4.3, the stay-away property (4.15) is crucial to being able to localize Theorems 4.7 and 4.8 to the setting of Riemannian manifolds. In addition, apart from this application, proving (or disproving) the validity of this property would represent an important step in the understanding of the geometry of optimal transportation.

(6) As explained in Section 4.4. there is a formal argument which suggests that the MTW condition should imply that all injectivity domains $\mathrm{I}(x)$ are convex for every $x \in M$. Proving this result in full generality would be interesting both for the regularity of optimal transport maps and as a purely geometric result.

(7) Another natural step in understanding the relation between the MTW condition and the regularity of optimal map would be to prove that the MTW condition and the convexity of the tangent cut-loci are both necessary and sufficient conditions for the continuity of optimal transport maps on Riemannian manifolds (with the cost given by the squared distance). As mentioned at the end of Section 4.4, only the necessity is currently known in general, while the sufficiency is known only in dimension 2. A nice problem would be to prove the sufficiency in every dimension. Notice that, if one could also prove (4) above, then as a corollary one would obtain that MTW is necessary and sufficient for regularity of optimal maps on any Riemannian manifold.

(8) It would be very interesting to give estimates on the Hausdorff dimension of the singular sets $\Sigma_{X}$ and $\Sigma_{Y}$ appearing in the partial regularity Theorems 4.11 and 4.12. In view of all known examples, one is led to conjecture that $\operatorname{dim}_{\mathcal{H}}\left(\Sigma_{X}\right), \operatorname{dim}_{\mathcal{H}}\left(\Sigma_{Y}\right) \leq n-1$. This is not known even in the case of the quadratic cost when the target is not convex, although some partial results in this direction have been obtained in [49]. An even more difficult problem would be to prove that the singular sets are always rectifiable or even, under suitable assumptions on the densities, smooth.

(9) Parabolic versions of the Monge-Ampère equation naturally appear in geometric evolution problems (see for instance 34 and the references therein). More recently, in [75, 76] the authors studied the parabolic version of (1.2) in the context of optimal transport, and they showed that, if the MTW condition holds, then under suitable conditions on the initial and target domains, the solution exists globally in time and converges exponentially fast, as $t \rightarrow \infty$ to the solution of the original mass transport problem. Apart

\footnotetext{
${ }^{14}$ To explain this with an example, one may think of covariant derivatives in classical Riemannian geometry: if instead of using them one just uses standard differentiation in charts, one would end up with complicated expressions which, by "magic", have a lot of simplifications. On the other hand by using covariant derivatives, formulas automatically simplify.
} 
from its own interest, this result provides a potential way to numerically solve the optimal transport problem by taking a smooth initial condition and running the parabolic flow for sufficiently long time. It would be extremely interesting to understand whether similar results may hold (in some weak sense) even without assuming the validity of the MTW condition, and then obtain partial regularity results in the spirit of Theorem 4.11.

(10) The assumption of the existence of a generating function $G$ ensures that the matrix $\mathcal{A}(x, u, \nabla u)$ appearing in (5.2) is symmetric. However, for the general class of equations (5.2) arising from the prescribed Jacobian equations (5.1), there is no reason why the matrix $\mathcal{A}$ should be symmetric. It would be very interesting to understand under which structural assumptions on $\mathcal{A}$ one can develop a regularity theory (see [101, Corollary 1.2] for a result in dimension 2).

\section{ACKNOWLEDGMEnTs}

The authors wish to thank Neil Trudinger for several useful comments on a preliminary version of this paper. The second author is partially supported by NSF Grant DMS-1262411. Both authors acknowledge the support of the ERC ADG Grant GeMeThNES.

\section{About The AUthors}

Guido De Philippis received his doctorate in 2012 from the Scuola Normale Superiore of Pisa, under the supervision of Luigi Ambrosio (SNS Pisa) and Luis Caffarelli (University of Texas at Austin). He is currently a postdoctoral fellow at the University of Zurich. He works in partial differential equations, calculus of variations, and geometric measure theory.

Alessio Figalli earned his doctorate in 2007 from the Scuola Normale Superiore of Pisa and the École Normale Supérieure of Lyon, under the supervision of Luigi Ambrosio (SNS Pisa) and Cédric Villani (ENS Lyon). He is currently professor and R. L. Moore Chair at the University of Texas at Austin. He has received several prizes, including the EMS prize in 2012. His main interests are in partial differential equations and calculus of variations.

\section{REFERENCES}

[1] H. Aimar, L. Forzani, and R. Toledano, Balls and quasi-metrics: a space of homogeneous type modeling the real analysis related to the Monge-Ampère equation, J. Fourier Anal. Appl. 4 (1998), no. 4-5, 377-381, DOI 10.1007/BF02498215. MR1658608 (99j:35043)

[2] A. Alexandroff, Existence and uniqueness of a convex surface with a given integral curvature, C. R. (Doklady) Acad. Sci. URSS (N.S.) 35 (1942), 131-134. MR0007625 (4,169c)

[3] A. Alexandroff, Smoothness of the convex surface of bounded Gaussian curvature, C. R. (Doklady) Acad. Sci. URSS (N.S.) 36 (1942), 195-199. MR0007626 (4,169d)

[4] A. D. Alexandrov, Convex polyhedra, Springer Monographs in Mathematics, Springer-Verlag, Berlin, 2005. Translated from the 1950 Russian edition by N. S. Dairbekov, S. S. Kutateladze and A. B. Sossinsky; With comments and bibliography by V. A. Zalgaller and appendices by L. A. Shor and Yu. A. Volkov. MR2127379 (2005j:52002)

[5] A. D. Alexandrov, Dirichlet's problem for the equation $\operatorname{det}\left\|z_{i j}\right\|=$ $\varphi\left(z_{1}, \ldots, z_{n}, z, x_{1}, \ldots, x_{n}\right)$ I. (Russian) Vestnik Leningrad. Univ. Ser. Mat. Meh. Astr. 13 (1958), no. 1, 5-24. 
[6] Luigi Ambrosio, Lecture notes on optimal transport problems, Mathematical aspects of evolving interfaces (Funchal, 2000), Lecture Notes in Math., vol. 1812, Springer, Berlin, 2003, pp. 1-52, DOI 10.1007/978-3-540-39189-0_1. MR2011032

[7] Luigi Ambrosio, Maria Colombo, Guido De Philippis, and Alessio Figalli, Existence of Eulerian solutions to the semigeostrophic equations in physical space: the 2-dimensional periodic case, Comm. Partial Differential Equations 37 (2012), no. 12, 2209-2227, DOI 10.1080/03605302.2012.669443. MR3005541

[8] Luigi Ambrosio, Maria Colombo, Guido De Philippis, Alessio Figalli, A global existence result for the semigeostrophic equations in three dimensional convex domains. Discrete Contin. Dyn. Syst., to appear.

[9] Luigi Ambrosio, Nicola Gigli, and Giuseppe Savaré, Gradient flows in metric spaces and in the space of probability measures, Lectures in Mathematics ETH Zürich, Birkhäuser Verlag, Basel, 2005. MR2129498(2006k:49001)

[10] A. M. Ampère, Mémoire contenant l'application de la théorie. Journal de l'École Polytechnique, (1820).

[11] Kari Astala, Tadeusz Iwaniec, and Gaven Martin, Elliptic partial differential equations and quasiconformal mappings in the plane, Princeton Mathematical Series, vol. 48, Princeton University Press, Princeton, NJ, 2009. MR2472875 (2010j:30040)

[12] I. Ya. Bakel'man, Generalized solutions of Monge-Ampère equations (Russian), Dokl. Akad. Nauk SSSR (N.S.) 114 (1957), 1143-1145. MR0095481 (20 \#1983)

[13] J.-D. Benamou and Y. Brenier, Weak existence for the semigeostrophic equations formulated as a coupled Monge-Ampère/transport problem, SIAM J. Appl. Math. 58 (1998), no. 5, 1450-1461 (electronic), DOI 10.1137/S0036139995294111. MR1627555(99i:35162)

[14] Yann Brenier, Décomposition polaire et réarrangement monotone des champs de vecteurs (French, with English summary), C. R. Acad. Sci. Paris Sér. I Math. 305 (1987), no. 19, 805-808. MR923203 (89b:58226)

[15] Yann Brenier, Polar factorization and monotone rearrangement of vector-valued functions, Comm. Pure Appl. Math. 44 (1991), no. 4, 375-417, DOI 10.1002/cpa.3160440402. MR 1100809 (92d:46088)

[16] L. A. Caffarelli, A localization property of viscosity solutions to the Monge-Ampère equation and their strict convexity, Ann. of Math. (2) 131 (1990), no. 1, 129-134, DOI 10.2307/1971509. MR 1038359 (91f:35058)

[17] Luis A. Caffarelli, Interior $W^{2, p}$ estimates for solutions of the Monge-Ampère equation, Ann. of Math. (2) 131 (1990), no. 1, 135-150, DOI 10.2307/1971510. MR 1038360 (91f:35059)

[18] Luis A. Caffarelli, Some regularity properties of solutions of Monge Ampère equation, Comm. Pure Appl. Math. 44 (1991), no. 8-9, 965-969, DOI 10.1002/cpa.3160440809. MR $1127042(92 \mathrm{~h}: 35088)$

[19] Luis A. Caffarelli, The regularity of mappings with a convex potential, J. Amer. Math. Soc. 5 (1992), no. 1, 99-104, DOI 10.2307/2152752. MR1124980(92j:35018)

[20] Luis A. Caffarelli, Boundary regularity of maps with convex potentials, Comm. Pure Appl. Math. 45 (1992), no. 9, 1141-1151, DOI 10.1002/cpa.3160450905. MR.1177479 (93k:35054)

[21] Luis A. Caffarelli, A note on the degeneracy of convex solutions to Monge Ampère equation, Comm. Partial Differential Equations 18 (1993), no. 7-8, 1213-1217, DOI 10.1080/03605309308820970. MR.1233191 (94f:35045)

[22] Luis A. Caffarelli, Boundary regularity of maps with convex potentials. II, Ann. of Math. (2) 144 (1996), no. 3, 453-496, DOI 10.2307/2118564. MR1426885 (97m:35027)

[23] Luis A. Caffarelli, M. M. Gonzáles, T. Nguyen, A perturbation argument for a MongeAmpère type equation arising in optimal transportation. Preprint.

[24] Luis A. Caffarelli and Cristian E. Gutiérrez, Real analysis related to the Monge-Ampère equation, Trans. Amer. Math. Soc. 348 (1996), no. 3, 1075-1092, DOI 10.1090/S0002-994796-01473-0. MR1321570 (96h:35047)

[25] Luis A. Caffarelli and Robert J. McCann, Free boundaries in optimal transport and MongeAmpère obstacle problems, Ann. of Math. (2) 171 (2010), no. 2, 673-730, DOI 10.4007/annals.2010.171.673. MR2630054 (2011b:49116)

[26] Eugenio Calabi, Improper affine hyperspheres of convex type and a generalization of a theorem by K. Jörgens, Michigan Math. J. 5 (1958), 105-126. MR0106487(21 \#5219) 
[27] Eugenio Calabi, Complete affine hyperspheres. I, Symposia Mathematica, Vol. X (Convegno di Geometria Differenziale, INDAM, Rome, 1971), Academic Press, London, 1972, pp. 19-38. MR 0365607 (51 \#1859)

[28] Piermarco Cannarsa and Carlo Sinestrari, Semiconcave functions, Hamilton-Jacobi equations, and optimal control, Progress in Nonlinear Differential Equations and their Applications, 58, Birkhäuser Boston, Inc., Boston, MA, 2004. MR2041617(2005e:49001)

[29] Shiu Yuen Cheng and Shing-Tung Yau, Complete affine hypersurfaces. I. The completeness of affine metrics, Comm. Pure Appl. Math. 39 (1986), no. 6, 839-866, DOI 10.1002/cpa.3160390606. MR859275 (87k:53127)

[30] Dario Cordero-Erausquin, Robert J. McCann, and Michael Schmuckenschläger, A Riemannian interpolation inequality à la Borell, Brascamp and Lieb, Invent. Math. 146 (2001), no. 2, 219-257, DOI 10.1007/s002220100160. MR1865396(2002k:58038)

[31] Juan Antonio Cuesta-Albertos and Carlos Matrán, Notes on the Wasserstein metric in Hilbert spaces, Ann. Probab. 17 (1989), no. 3, 1264-1276. MR.1009457 (90k:60029)

[32] L. Caffarelli, L. Nirenberg, and J. Spruck, The Dirichlet problem for nonlinear second-order elliptic equations. I. Monge-Ampère equation, Comm. Pure Appl. Math. 37 (1984), no. 3, 369-402, DOI 10.1002/cpa.3160370306. MR739925 (87f:35096)

[33] M. Cullen, A mathematical theory of large-scale atmosphere/ocean flow. Imperial College Press (2006).

[34] Panagiota Daskalopoulos and Ovidiu Savin, $C^{1, \alpha}$ regularity of solutions to parabolic Monge-Ampére equations, Amer. J. Math. 134 (2012), no. 4, 1051-1087, DOI 10.1353/ajm.2012.0030. MR2956257

[35] Guido De Philippis and Alessio Figalli, $W^{2,1}$ regularity for solutions of the MongeAmpère equation, Invent. Math. 192 (2013), no. 1, 55-69, DOI 10.1007/s00222-012-0405-4. MR 3032325

[36] Guido De Philippis and Alessio Figalli, Second order stability for the Monge-Ampère equation and strong Sobolev convergence of optimal transport maps, Anal. PDE 6 (2013), no. 4, 993-1000, DOI 10.2140/apde.2013.6.993. MR3092736

[37] Guido De Philippis and Alessio Figalli, Sobolev regularity for Monge-Ampère type equations, SIAM J. Math. Anal. 45 (2013), no. 3, 1812-1824, DOI 10.1137/120898619. MR3066801

[38] Guido De Philippis, Alessio Figalli, Optimal regularity of the convex envelope. Preprint.

[39] Guido De Philippis, Alessio Figalli, Partial regularity for optimal transport maps. Preprint.

[40] G. De Philippis, A. Figalli, and O. Savin, A note on interior $W^{2,1+\varepsilon}$ estimates for the Monge-Ampère equation, Math. Ann. 357 (2013), no. 1, 11-22, DOI 10.1007/s00208-0120895-9. MR3084340

[41] Philippe Delanoë and Yuxin Ge, Regularity of optimal transport on compact, locally nearly spherical, manifolds, J. Reine Angew. Math. 646 (2010), 65-115, DOI 10.1515/CRELLE.2010.066. MR2719556 (2012e:49093)

[42] Philippe Delanoë and Grégoire Loeper, Gradient estimates for potentials of invertible gradient-mappings on the sphere, Calc. Var. Partial Differential Equations 26 (2006), no. 3, 297-311, DOI 10.1007/s00526-006-0006-4. MR2232207 (2007b:35108)

[43] Lawrence C. Evans, Classical solutions of fully nonlinear, convex, second-order elliptic equations, Comm. Pure Appl. Math. 35 (1982), no. 3, 333-363, DOI 10.1002/cpa.3160350303. MR:649348(83g:35038)

[44] Lawrence C. Evans and Ronald F. Gariepy, Measure theory and fine properties of functions, Studies in Advanced Mathematics, CRC Press, Boca Raton, FL, 1992. MR 1158660 (93f:28001)

[45] Albert Fathi and Alessio Figalli, Optimal transportation on non-compact manifolds, Israel J. Math. 175 (2010), 1-59, DOI 10.1007/s11856-010-0001-5. MR2607536 (2011c:49091)

[46] Herbert Federer, Geometric measure theory, Die Grundlehren der mathematischen Wissenschaften, Band 153, Springer-Verlag New York Inc., New York, 1969. MR0257325 (41 \#1976)

[47] Alessio Figalli, A note on the regularity of the free boundaries in the optimal partial transport problem, Rend. Circ. Mat. Palermo (2) 58 (2009), no. 2, 283-286, DOI 10.1007/s12215-0090022-2. MR 2533917 (2010m:49064)

[48] Alessio Figalli, The optimal partial transport problem, Arch. Ration. Mech. Anal. 195 (2010), no. 2, 533-560, DOI 10.1007/s00205-008-0212-7. MR2592287 (2011f:49069) 
[49] Alessio Figalli, Regularity properties of optimal maps between nonconvex domains in the plane, Comm. Partial Differential Equations 35 (2010), no. 3, 465-479, DOI 10.1080/03605300903307673. MR2748633 (2012f:35215)

[50] Alessio Figalli, Sobolev regularity for the Monge-Ampère equation, with application to the semigeostrophic equations (English, with English and Russian summaries), Zap. Nauchn. Sem. S.-Peterburg. Otdel. Mat. Inst. Steklov. (POMI) 411 (2013), no. Teoriya Predstavlenii, Dinamicheskie Sistemy, Kombinatornye Metody. XXII, 103-118, 242. MR 3048271

[51] Alessio Figalli and Young-Heon Kim, Partial regularity of Brenier solutions of the Monge-Ampère equation, Discrete Contin. Dyn. Syst. 28 (2010), no. 2, 559-565, DOI 10.3934/dcds.2010.28.559. MR2644756(2011d:35158)

[52] Alessio Figalli, Young-Heon Kim, and Robert J. McCann, Hölder continuity and injectivity of optimal maps, Arch. Ration. Mech. Anal. 209 (2013), no. 3, 747-795, DOI 10.1007/s00205013-0629-5. MR3067826

[53] Alessio Figalli, Young-Heon Kim, and Robert J. McCann, Regularity of optimal transport maps on multiple products of spheres, J. Eur. Math. Soc. (JEMS) 15 (2013), no. 4, 11311166, DOI 10.4171/JEMS/388. MR3055758

[54] Alessio Figalli and Grégoire Loeper, $C^{1}$ regularity of solutions of the Monge-Ampère equation for optimal transport in dimension two, Calc. Var. Partial Differential Equations 35 (2009), no. 4, 537-550, DOI 10.1007/s00526-009-0222-9. MR.2496656(2010g:35128)

[55] Alessio Figalli and Ludovic Rifford, Continuity of optimal transport maps and convexity of injectivity domains on small deformations of $\mathbb{S}^{2}$, Comm. Pure Appl. Math. 62 (2009), no. 12, 1670-1706, DOI 10.1002/cpa.20293. MR2569074(2010k:49094)

[56] Alessio Figalli, Ludovic Rifford, and Cédric Villani, On the Ma-Trudinger-Wang curvature on surfaces, Calc. Var. Partial Differential Equations 39 (2010), no. 3-4, 307-332, DOI 10.1007/s00526-010-0311-9. MR2729302 (2012d:53106)

[57] A. Figalli, L. Rifford, and C. Villani, Nearly round spheres look convex, Amer. J. Math. 134 (2012), no. 1, 109-139, DOI 10.1353/ajm.2012.0000. MR2876141

[58] Alessio Figalli, Ludovic Rifford, and Cédric Villani, Necessary and sufficient conditions for continuity of optimal transport maps on Riemannian manifolds, Tohoku Math. J. (2) 63 (2011), no. 4, 855-876, DOI 10.2748/tmj/1325886291. MR2872966

[59] Liliana Forzani and Diego Maldonado, Properties of the solutions to the Monge-Ampère equation, Nonlinear Anal. 57 (2004), no. 5-6, 815-829, DOI 10.1016/j.na.2004.03.019. MR 2067735(2005c:35095)

[60] Ilaria Fragalà, Maria Stella Gelli, and Aldo Pratelli, Continuity of an optimal transport in Monge problem (English, with English and French summaries), J. Math. Pures Appl. (9) 84 (2005), no. 9, 1261-1294, DOI 10.1016/j.matpur.2005.02.002. MR2162225(2006g:49074)

[61] Wilfrid Gangbo and Robert J. McCann, The geometry of optimal transportation, Acta Math. 177 (1996), no. 2, 113-161, DOI 10.1007/BF02392620. MR1440931(98e:49102)

[62] David Gilbarg and Neil S. Trudinger, Elliptic partial differential equations of second order, 2nd ed., Grundlehren der Mathematischen Wissenschaften [Fundamental Principles of Mathematical Sciences], vol. 224, Springer-Verlag, Berlin, 1983. MR737190 (86c:35035)

[63] N. Guillen, J. Kitagawa, The local geometry of maps with c-convex potentials. Preprint.

[64] Cristian E. Gutiérrez, The Monge-Ampère equation, Progress in Nonlinear Differential Equations and their Applications, 44, Birkhäuser Boston, Inc., Boston, MA, 2001. MR $1829162(2002 \mathrm{e}: 35075)$

[65] Cristian E. Gutiérrez and Qingbo Huang, Geometric properties of the sections of solutions to the Monge-Ampère equation, Trans. Amer. Math. Soc. 352 (2000), no. 9, 4381-4396, DOI 10.1090/S0002-9947-00-02491-0. MR1665332(2000m:35060)

[66] Emanuel Indrei, Free boundary regularity in the optimal partial transport problem, J. Funct. Anal. 264 (2013), no. 11, 2497-2528, DOI 10.1016/j.jfa.2013.03.006. MR3041707

[67] Jin-ichi Itoh and Minoru Tanaka, The Lipschitz continuity of the distance function to the cut locus, Trans. Amer. Math. Soc. 353 (2001), no. 1, 21-40, DOI 10.1090/S0002-9947-0002564-2. MR1695025 (2001b:53029)

[68] N. M. Ivochkina, Classical solvability of the Dirichlet problem for the Monge-Ampère equation (Russian, with English summary), Zap. Nauchn. Sem. Leningrad. Otdel. Mat. Inst. Steklov. (LOMI) 131 (1983), 72-79. Questions in quantum field theory and statistical physics, 4. MR718679 (85g:35044) 
[69] Fritz John, Extremum problems with inequalities as subsidiary conditions, Studies and Essays Presented to R. Courant on his 60th Birthday, January 8, 1948, Interscience Publishers, Inc., New York, N. Y., 1948, pp. 187-204. MR0030135(10,719b)

[70] L. V. Kantorovich, On mass transportation (Russian), Zap. Nauchn. Sem. S.-Peterburg. Otdel. Mat. Inst. Steklov. (POMI) 312 (2004), no. Teor. Predst. Din. Sist. Komb. i Algoritm. Metody. 11, 11-14, DOI 10.1007/s10958-006-0049-2; English transl., J. Math. Sci. (N. Y.) 133 (2006), no. 4, 1381-1382. MR2117876

[71] L. V. Kantorovich, On a problem of Monge (Russian), Zap. Nauchn. Sem. S.-Peterburg. Otdel. Mat. Inst. Steklov. (POMI) 312 (2004), no. Teor. Predst. Din. Sist. Komb. i Algoritm. Metody. 11, 15-16, DOI 10.1007/s10958-006-0050-9; English transl., J. Math. Sci. (N. Y.) 133 (2006), no. 4, 1383. MR2117877

[72] Young-Heon Kim, Counterexamples to continuity of optimal transport maps on positively curved Riemannian manifolds, Int. Math. Res. Not. IMRN, posted on 2008, Art. ID rnn120, 15, DOI 10.1093/imrn/rnn120. MR2448078 (2009j:53037)

[73] Young-Heon Kim and Robert J. McCann, Continuity, curvature, and the general covariance of optimal transportation, J. Eur. Math. Soc. (JEMS) 12 (2010), no. 4, 1009-1040, DOI 10.4171/JEMS/221. MR2654086(2011f:49071)

[74] Young-Heon Kim and Robert J. McCann, Towards the smoothness of optimal maps on Riemannian submersions and Riemannian products (of round spheres in particular), J. Reine Angew. Math. 664 (2012), 1-27, DOI 10.1515/CRELLE.2011.105. MR2980128

[75] Young-Heon Kim, Jeffrey Streets, and Micah Warren, Parabolic optimal transport equations on manifolds, Int. Math. Res. Not. IMRN 19 (2012), 4325-4350, DOI 10.1093/imrn/rnr188. MR2981711

[76] Jun Kitagawa, A parabolic flow toward solutions of the optimal transportation problem on domains with boundary, J. Reine Angew. Math. 672 (2012), 127-160. MR2995434

[77] N. V. Krylov, Boundedly inhomogeneous elliptic and parabolic equations (Russian), Izv. Akad. Nauk SSSR Ser. Mat. 46 (1982), no. 3, 487-523, 670. MR661144 (84a:35091)

[78] N. V. Krylov, Boundedly inhomogeneous elliptic and parabolic equations in a domain (Russian), Izv. Akad. Nauk SSSR Ser. Mat. 47 (1983), no. 1, 75-108. MR688919(85g:35046)

[79] Q. R. Li, F. Santambrogio, X. J. Wang, Regularity in Monge's mass transfer problem. Preprint.

[80] Jiakun Liu, Hölder regularity of optimal mappings in optimal transportation, Calc. Var. Partial Differential Equations 34 (2009), no. 4, 435-451, DOI 10.1007/s00526-008-0190-5. MR:2476419(2010b:35130)

[81] Jiakun Liu, Neil S. Trudinger, and Xu-Jia Wang, Interior $C^{2, \alpha}$ regularity for potential functions in optimal transportation, Comm. Partial Differential Equations 35 (2010), no. 1, 165-184, DOI 10.1080/03605300903236609. MR2748621 (2011m:35095)

[82] Jiakun Liu, Neil S. Trudinger, Xu-Jia Wang, On asymptotic behaviour and $W^{2, p}$ regularity of potentials in optimal transportation. Preprint.

[83] G. Loeper, A fully nonlinear version of the incompressible Euler equations: the semigeostrophic system, SIAM J. Math. Anal. 38 (2006), no. 3, 795-823 (electronic), DOI 10.1137/050629070. MR2262943 (2007j:35173)

[84] Grégoire Loeper, On the regularity of solutions of optimal transportation problems, Acta Math. 202 (2009), no. 2, 241-283, DOI 10.1007/s11511-009-0037-8. MR2506751 (2010c:49084)

[85] Grégoire Loeper, Regularity of optimal maps on the sphere: the quadratic cost and the reflector antenna, Arch. Ration. Mech. Anal. 199 (2011), no. 1, 269-289, DOI 10.1007/s00205010-0330-x. MR2754343 (2011j:49081)

[86] Grégoire Loeper and Cédric Villani, Regularity of optimal transport in curved geometry: the nonfocal case, Duke Math. J. 151 (2010), no. 3, 431-485, DOI 10.1215/00127094-2010-003. MR2605867(2011f:49073)

[87] Xi-Nan Ma, Neil S. Trudinger, and Xu-Jia Wang, Regularity of potential functions of the optimal transportation problem, Arch. Ration. Mech. Anal. 177 (2005), no. 2, 151-183, DOI 10.1007/s00205-005-0362-9. MR 2188047 (2006m:35105)

[88] Robert J. McCann, Polar factorization of maps on Riemannian manifolds, Geom. Funct. Anal. 11 (2001), no. 3, 589-608, DOI 10.1007/PL00001679. MR1844080 (2002g:58017) 
[89] Hermann Minkowski, Allgemeine Lehrsätze über die konvexen Polyeder. Nachr. Ges. Wiss. Göttingen, (1897), 198-219.

[90] Hermann Minkowski, Volumen und Oberfläche (German), Math. Ann. 57 (1903), no. 4, 447-495, DOI 10.1007/BF01445180. MR1511220

[91] G. Monge, Mémoire sur la Théorie des Déblais et des Remblais. Hist. de l'Acad. des Sciences de Paris (1781), 666-704.

[92] G. Monge, Sur le calcul intégral des équations aux differences partielles. Mémoires de l'Académie des Sciences (1784).

[93] C. Mooney, Partial regularity for singular solutions to the Monge-Ampère equation. Comm. Pure Appl. Math., to appear.

[94] A. V. Pogorelov, The regularity of the generalized solutions of the equation $\operatorname{det}\left(\partial^{2} u / \partial x^{i} \partial x^{j}\right)=\varphi\left(x^{1}, x^{2}, \ldots, x^{n}\right)>0$ (Russian), Dokl. Akad. Nauk SSSR 200 (1971), 534-537. MR0293227(45 \#2304)

[95] A. V. Pogorelov, On the improper convex affine hyperspheres, Geometriae Dedicata 1 (1972), no. 1, 33-46. MR0319126 (47 \#7672)

[96] L. Rüschendorf and S. T. Rachev, A characterization of random variables with minimum $L^{2}$ distance, J. Multivariate Anal. 32 (1990), no. 1, 48-54, DOI 10.1016/0047-259X(90)90070-X. MR 1035606 (91e:60041a)

[97] Ovidiu Savin, A localization property at the boundary for Monge-Ampère equation, Advances in geometric analysis, Adv. Lect. Math. (ALM), vol. 21, Int. Press, Somerville, MA, 2012, pp. 45-68. MR3077247

[98] O. Savin, Pointwise $C^{2, \alpha}$ estimates at the boundary for the Monge-Ampère equation, J. Amer. Math. Soc. 26 (2013), no. 1, 63-99, DOI 10.1090/S0894-0347-2012-00747-4. MR.2983006

[99] O. Savin, Global $W^{2, p}$ estimates for the Monge-Ampère equation, Proc. Amer. Math. Soc. 141 (2013), no. 10, 3573-3578, DOI 10.1090/S0002-9939-2013-11748-X. MR3080179

[100] Thomas Schmidt, $\mathrm{W}^{2,1+\varepsilon}$ estimates for the Monge-Ampère equation, Adv. Math. 240 (2013), 672-689, DOI 10.1016/j.aim.2012.07.034. MR3046322

[101] Neil S. Trudinger, On the prescribed Jacobian equation. Proceedings of International Conference for the 25th Anniversary of Viscosity Solutions, 243-255, GAKUTO Internat. Ser. Math. Sci. Appl. 30, Gakkotosho, Tokyo, 2008.

[102] Neil S. Trudinger, On the local theory of prescribed Jacobian equations, Discrete Contin. Dyn. Syst. 34 (2014), no. 4, 1663-1681, DOI 10.3934/dcds.2014.34.1663. MR3121636

[103] Neil S. Trudinger, A note on global regularity in optimal transportion, Bull. Math. Sci. 3 (2013), no. 3, 551-557, DOI 10.1007/s13373-013-0046-y. MR3128040

[104] Neil S. Trudinger and Xu-Jia Wang, The Bernstein problem for affine maximal hypersurfaces, Invent. Math. 140 (2000), no. 2, 399-422, DOI 10.1007/s002220000059. MR1757001 (2001h:53016)

[105] Neil S. Trudinger and Xu-Jia Wang, Affine complete locally convex hypersurfaces, Invent. Math. 150 (2002), no. 1, 45-60, DOI 10.1007/s00222-002-0229-8. MR1930881(2003h:53012)

[106] Neil S. Trudinger and Xu-Jia Wang, The affine Plateau problem, J. Amer. Math. Soc. 18 (2005), no. 2, 253-289, DOI 10.1090/S0894-0347-05-00475-3. MR2137978 (2006e:53071)

[107] Neil S. Trudinger and Xu-Jia Wang, The Monge-Ampère equation and its geometric applications, Handbook of geometric analysis. No. 1, Adv. Lect. Math. (ALM), vol. 7, Int. Press, Somerville, MA, 2008, pp. 467-524. MR2483373 (2010g:53065)

[108] Neil S. Trudinger and Xu-Jia Wang, On the second boundary value problem for MongeAmpère type equations and optimal transportation, Ann. Sc. Norm. Super. Pisa Cl. Sci. (5) 8 (2009), no. 1, 143-174. MR2512204(2011b:49121)

[109] Neil S. Trudinger and Xu-Jia Wang, On strict convexity and continuous differentiability of potential functions in optimal transportation, Arch. Ration. Mech. Anal. 192 (2009), no. 3, 403-418, DOI 10.1007/s00205-008-0147-z. MR2505359(2010c:49085)

[110] Cédric Villani, Optimal transport, old and new, Grundlehren der Mathematischen Wissenschaften [Fundamental Principles of Mathematical Sciences], vol. 338, Springer-Verlag, Berlin, 2009. MR2459454 (2010f:49001)

[111] Xu Jia Wang, Some counterexamples to the regularity of Monge-Ampère equations, Proc. Amer. Math. Soc. 123 (1995), no. 3, 841-845, DOI 10.2307/2160809. MR1223269 (95d:35025) 
[112] Xu-Jia Wang, On the design of a reflector antenna, Inverse Problems 12 (1996), no. 3, 351-375, DOI 10.1088/0266-5611/12/3/013. MR1391544(97c:78014)

[113] Xu-Jia Wang, Strict convexity and $C^{1, \alpha}$ regularity of potential functions in optimal transportation under condition A3w. Preprint.

Institut FÜr MAthematik Universität ZÜRICH, Winterthurerstr. 190, CH-8057 Zürich (SWITZERLAND)

E-mail address: guido.dephilippis@math.uzh.ch

The University of Texas at Austin, Mathematics Dept. RLM 8.100, 2515 Speedway Stop C1200, Austin, Texas 78712-1202

E-mail address: figalli@math.utexas.edu 